\title{
Radical cyclizations for the synthesis of pyrroloindoles: Progress towards the flinderoles
}

\author{
Joanne E. Curiel Tejeda, Bryan K. Landschoot and Michael A. Kerr* \\ Department of Chemistry, the University of Western Ontario \\ London, Ontario, Canada N6A 5B7
}

Supporting Information 


\section{General information}

All reactions were carried under an Argon atmosphere unless indicated. Toluene, tetrahydrofuran (THF), $\mathrm{N}, \mathrm{N}$-dimethylformamide (DMF), and dichloromethane $\left(\mathrm{CH}_{2} \mathrm{Cl}_{2}\right)$ were dried and deoxygenated by passing the nitrogen purged solvents through activated alumina columns. All other reagents and solvents were used as purchased from Sigma Aldrich, Caledon or VWR. Reaction progress was followed by thin layer chromatography (TLC) (EM Science, silica gel $60 \mathrm{~F}_{254}$ ) visualizing with UV light, and the plates were developed using acidic anisaldehyde. Flash chromatography was performed using silica gel purchased from Silicycle Chemical Division Inc. (230-400 mesh).

NMR experiments were performed on the Varian Mercury 400 and Inova 400 instruments; samples were obtained in $\mathrm{CDCl}_{3}$ (referenced to $7.26 \mathrm{ppm}$ for ${ }^{1} \mathrm{H}$ and $77.0 \mathrm{ppm}$ for ${ }^{13} \mathrm{C}$ ). Coupling constants $(\mathcal{J})$ are in $\mathrm{Hz}$. The multiplicities of the signals are described using the following abbreviations: $\mathrm{s}=$ singlet, $\mathrm{d}=$ doublet, $\mathrm{t}=$ triplet, $\mathrm{q}=$ quartet, $\mathrm{m}=$ multiplet, $\mathrm{b}=$ broad. Infrared spectra were obtained as thin films on $\mathrm{NaCl}$ plates using the Brucker Vector $33 \mathrm{FT}$-IR instrument. High resolution mass spectra (HRMS) were obtained on a Finnigan MAT 8200 spectrometer at $70 \mathrm{eV}$. Melting points were determined using a Gallenkamp melting point apparatus and were uncorrected. Microwave reactions were performed in a $400 \mathrm{~W}$ Biotage Initiator 2.0 microwave reactor.

\section{Synthesis of compound 4kk}

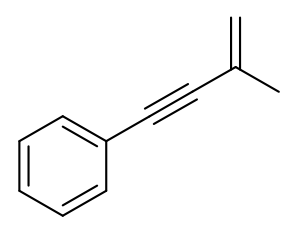

\section{(3-methylbut-3-en-1-yn-1-yl)benzene (4kk).}

Commercially available phenylacetylene (100 mg, $0.980 \mathrm{mmol}, 1$ equiv) was dissolved in $5 \mathrm{~mL}$ of dry $\mathrm{Et}_{3} \mathrm{~N}$, followed by the addition of commercially available 2-bromoprop-1-ene (142 mg, $1.18 \mathrm{mmol}, 1.2$ equivs), copper iodide (Cul) (95 mg, $0.050 \mathrm{mmol}, 5 \mathrm{~mol} \%)$, and $\mathrm{Pd}\left(\mathrm{PPh}_{3}\right)_{4}(29 \mathrm{mg}, 0.025 \mathrm{mmol}, 2.5 \mathrm{~mol} \%)$. The reaction mixture was heated to $90{ }^{\circ} \mathrm{C}$ for 12 hours. The reaction was cooled to room temperature and the solvent was removed in vacuo. The crude product was dissolved in $20 \mathrm{~mL}$ of $\mathrm{CH}_{2} \mathrm{Cl}_{2}$ and washed with water $(2 \times 10 \mathrm{~mL})$. The organic portion was dried over $\mathrm{MgSO}_{4}$, filtered, and concentrated. Purification of the crude reaction mixture by column chromatography gave the title compound ( $84 \mathrm{mg}, 0.587 \mathrm{mmol}, 60 \%$ ) as colourless oil. Spectral data for this compound matched the previously reported ${ }^{1}$. 


\section{Experimental Procedure A: Synthesis of Quaternary D-A Cyclopropanes}

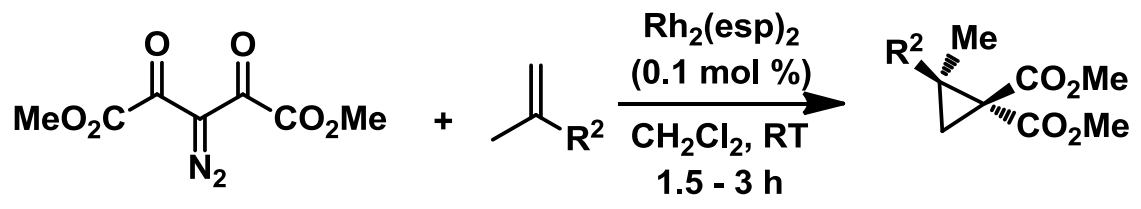

Cyclopropanes 4i-m were prepared according to the following procedure. In a $10 \mathrm{~mL}$ or $25 \mathrm{~mL}$ round-bottomed flask was added the corresponding 2-methyl-1-hexen-3-yne derivative (1.0 equiv), $\mathrm{CH}_{2} \mathrm{Cl}_{2}(4 \mathrm{~mL}-8 \mathrm{~mL})$ and $\mathrm{Rh}_{2}(\mathrm{esp})_{2}$ catalyst $(0.1 \mathrm{~mol} \%)$. The diazomalonate $(1.3$ equivs) was dissolved in $\mathrm{CH}_{2} \mathrm{Cl}_{2}(3 \mathrm{~mL})$ and added dropwise over a period of 45 mins $-1 \mathrm{~h}$ at room temperature. The reaction was stirred at room temperature for $1.5-3 \mathrm{~h}$ (monitored by TLC). The crude reaction mixture was concentrated, pre-absorbed onto silica gel, and purified by column chromatography (EtOAc in Hexanes).

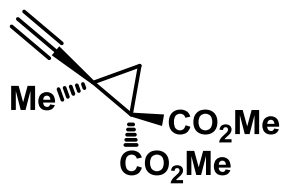

\section{Dimethyl 2-ethynyl-2-methylcyclopropane-1,1-dicarboxylate (4i).}

Following experimental procedure $A$, cyclopropane 4i was prepared by dissolving commercially available 2-methylbut-1-en-3-yne (500 mg, 7.56 $\mathrm{mmol}$ ) and $\mathrm{Rh}_{2}(\mathrm{esp})_{2}(5.73 \mathrm{mg}, 0.008 \mathrm{mmol})$ in $5 \mathrm{~mL}$ of $\mathrm{CH}_{2} \mathrm{Cl}_{2}$ followed by the addition of diazomalonate (1055 mg, $9.82 \mathrm{mmol}$ ) dissolved in $3 \mathrm{~mL}$ of $\mathrm{CH}_{2} \mathrm{Cl}_{2}$. The reaction was stirred at room temperature for $2.5 \mathrm{~h}$. Cyclopropane $4 \mathbf{i}(518 \mathrm{mg}, 2.64 \mathrm{mmol}, 35 \%)$ was obtained as a colourless oil. $\mathrm{Rf}=0.47\left(30 \%\right.$ EtOAc in hexanes). ${ }^{1} \mathrm{HNMR}\left(400 \mathrm{MHz}, \mathrm{CDCl}_{3}\right): \delta=3.78(\mathrm{~s}, 6 \mathrm{H})$, $2.09(\mathrm{~s}, 1 \mathrm{H}), 1.94(\mathrm{~d}, J=5.0 \mathrm{~Hz}, 1 \mathrm{H}), 1.60(\mathrm{~d}, J=5.0 \mathrm{~Hz}, 1 \mathrm{H}), 1.47(\mathrm{~s}, 3 \mathrm{H}) .{ }^{13} \mathrm{CNMR}(100$ $\mathrm{MHz}, \mathrm{CDCl}_{3}$ ): $\delta=167.1,167.0,83.5,68.3,52.5,39.9,26.8,22.8,19.9$. FT-IR (thin film, $\mathrm{cm}^{-1}$ ): $3280,3001,2954,2117,1738,1436,1335,1250,1118$. HRMS calc'd for $\mathrm{C}_{10} \mathrm{H}_{12} \mathrm{O}_{4}\left[\mathrm{M}^{+}\right]$: 196.0736; found: 196.0733 .

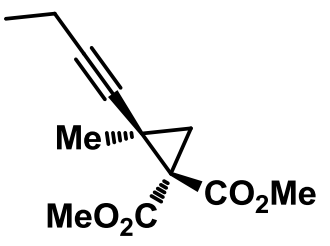

Dimethyl 2-(but-1-ynyl)-2-methylcyclopropane-1,1-dicarboxylate (4j). Following experimental procedure A, cyclopropane $\mathbf{4 j}$ was prepared by dissolving commercially available 2-methylhex-1-en-3-yne (500 mg, 5.31 $\mathrm{mmol}$ ) and $\mathrm{Rh}_{2}(\mathrm{esp})_{2}\left(4.02 \mathrm{mg}, 0.005 \mathrm{mmol}\right.$ ) in $5 \mathrm{~mL}$ of $\mathrm{CH}_{2} \mathrm{Cl}_{2}$ followed by the addition of diazomalonate $\left(1090 \mathrm{mg}, 6.90 \mathrm{mmol}\right.$ ) dissolved in $3 \mathrm{~mL}$ of $\mathrm{CH}_{2} \mathrm{Cl}_{2}$. The reaction was stirred at room temperature for $3 \mathrm{~h}$. Cyclopropane $4 \mathrm{j}$ (1010 mg, $4.50 \mathrm{mmol}, 85 \%)$ was obtained as a colourless oil. Rf $=0.51$ (30\% EtOAc in hexanes). ${ }^{1} \mathrm{HNMR}$ (400 MHz, $\left.\mathrm{CDCl}_{3}\right): \delta=3.68(\mathrm{~s}, 3 \mathrm{H}), 3.67(\mathrm{~s}, 3 \mathrm{H}), 2.04(\mathrm{q}, J=7.8 \mathrm{~Hz}, 2 \mathrm{H}), 1.79(\mathrm{~d}, J=4.7 \mathrm{~Hz}, 1 \mathrm{H}), 1.47(\mathrm{~d}$, 
$J=4.7 \mathrm{~Hz}, 1 \mathrm{H}), 1.35(\mathrm{~s}, 3 \mathrm{H}), 0.99(\mathrm{t}, J=7.8 \mathrm{~Hz}, 3 \mathrm{H}) .{ }^{13} \mathrm{CNMR}\left(100 \mathrm{MHz}, \mathrm{CDCl}_{3}\right): \delta=167.6$, 167.3, 82.0, 79.2, 52.5, 52.4, 40.3, 27.1, 23.9, 20.4, 13.7, 12.1. FT-IR (thin film, $\mathrm{cm}^{-1}$ ): 2976, 2933, 2128, 1739, 1436, 1329, 1234, 1119. HRMS calc'd for $\mathrm{C}_{12} \mathrm{H}_{16} \mathrm{O}_{4}\left[\mathrm{M}^{+}\right]$: 224.1049; found: 224.1053.

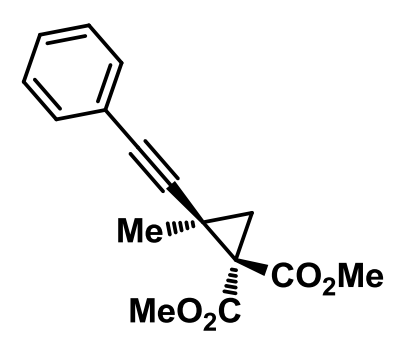

\section{Dimethyl 2-methyl-2-(phenylethynyl)cyclopropane-1,1-dicarboxylate} (4k). Following experimental procedure $A$, cyclopropane $\mathbf{4 k}$ was prepared by dissolving (3-methylbut-3-en-1-yn-1-yl)benzene 4kk (395 mg, 2.78 mmol) and $\mathrm{Rh}_{2}(\mathrm{esp})_{2}$ (2.10 mg, $0.003 \mathrm{mmol}$ ) in $3 \mathrm{~mL}$ of $\mathrm{CH}_{2} \mathrm{Cl}_{2}$ followed by the addition of diazomalonate $(571 \mathrm{mg}, 3.61 \mathrm{mmol}$ ) dissolved in $3 \mathrm{~mL}$ of $\mathrm{CH}_{2} \mathrm{Cl}_{2}$. The reaction was stirred at room temperature for $1.5 \mathrm{~h}$. Cyclopropane $4 \mathbf{k}$ (707 $\mathrm{mg}$, $2.59 \mathrm{mmol}, 93 \%)$ was obtained as a colourless oil. $\mathrm{Rf}=0.51$ (30\% EtOAc in hexanes). ${ }^{1} \mathrm{HNMR}$ $\left(400 \mathrm{MHz}, \mathrm{CDCl}_{3}\right): \delta=7.35$ (dd, $\left.J=5.9,2.3 \mathrm{~Hz}, 2 \mathrm{H}\right), 7.25(\mathrm{~m}, 3 \mathrm{H}), 3.77(\mathrm{~s}, 3 \mathrm{H}), 3.75(\mathrm{~s}, 3 \mathrm{H})$, $2.03(\mathrm{~d}, J=5.0 \mathrm{~Hz}, 1 \mathrm{H}), 1.69(\mathrm{~d}, J=5.0 \mathrm{~Hz}, 1 \mathrm{H}), 1.54(\mathrm{~s}, 3 \mathrm{H}) .{ }^{13} \mathrm{CNMR}\left(100 \mathrm{MHz}, \mathrm{CDCl}_{3}\right): \delta=$ $167.7,167.4,131.6,128.2,128.0,122.8,89.5,80.5,52.8,40.9,27.5,24.3,20.2$. FT-IR (thin film, $\left.\mathrm{cm}^{-1}\right): \quad 2998,2952,2198,1736,1435,1237,1116$. HRMS calc'd for $\mathrm{C}_{16} \mathrm{H}_{16} \mathrm{O}_{4}\left[\mathrm{M}^{+}\right]$: 272.1049; found: 272.1056 .

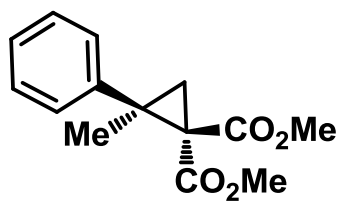

\section{Dimethyl 2-methyl-2-phenylcyclopropane-1,1-dicarboxylate (4I).}

Following experimental procedure A, cyclopropane 4I was prepared by dissolving commercially available $\alpha$-methylstyrene $(500 \mathrm{mg}, 4.23 \mathrm{mmol}$ ) and $\mathrm{Rh}_{2}$ (esp) $)_{2}\left(3.20 \mathrm{mg}, 0.004 \mathrm{mmol}\right.$ ) in $5 \mathrm{~mL}$ of $\mathrm{CH}_{2} \mathrm{Cl}_{2}$ followed by the addition of diazomalonate (1030 mg, $6.34 \mathrm{mmol}$ ) dissolved in $3 \mathrm{~mL}$ of $\mathrm{CH}_{2} \mathrm{Cl}_{2}$. The reaction was stirred at room temperature for $3 \mathrm{~h}$. Cyclopropane $4 \mathrm{l}(1030 \mathrm{mg}, 4.15 \mathrm{mmol}, 98 \%)$ was obtained as colourless oil. $\mathrm{Rf}=0.56$ (30\% EtOAc in hexanes). Spectral data for this compound matched the previously reported ${ }^{2}$.

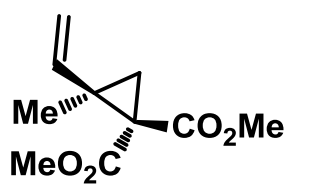

Dimethyl 2-methyl-2-vinylcyclopropane-1,1-dicarboxylate (4m).

Following experimental procedure A, cyclopropane $4 \mathrm{~m}$ was prepared by dissolving commercially available isoprene $(500 \mathrm{mg}, 7.34 \mathrm{mmol})$ and $\mathrm{Rh}_{2}$ (esp) $)_{2}\left(5.57 \mathrm{mg}, 0.007 \mathrm{mmol}\right.$ ) in $5 \mathrm{~mL}$ of $\mathrm{CH}_{2} \mathrm{Cl}_{2}$ followed by the addition of diazomalonate (1740 mg, $11.01 \mathrm{mmol}$ ) dissolved in $3 \mathrm{~mL}$ of $\mathrm{CH}_{2} \mathrm{Cl}_{2}$. The reaction was stirred at room temperature for $3 \mathrm{~h}$. Cyclopropane $\mathbf{4 m}$ (754 mg, $3.80 \mathrm{mmol}, 52 \%$ ) was obtained as a 67:33 
inseparable mixture of isomers and as a colourless oil. $\mathrm{Rf}=0.56$ (30\% EtOAc in hexanes). Spectral data for this compound matched the previously reported ${ }^{2}$.

\section{Experimental Procedure B: Indoline Ring Opening of D-A Cyclopropanes}

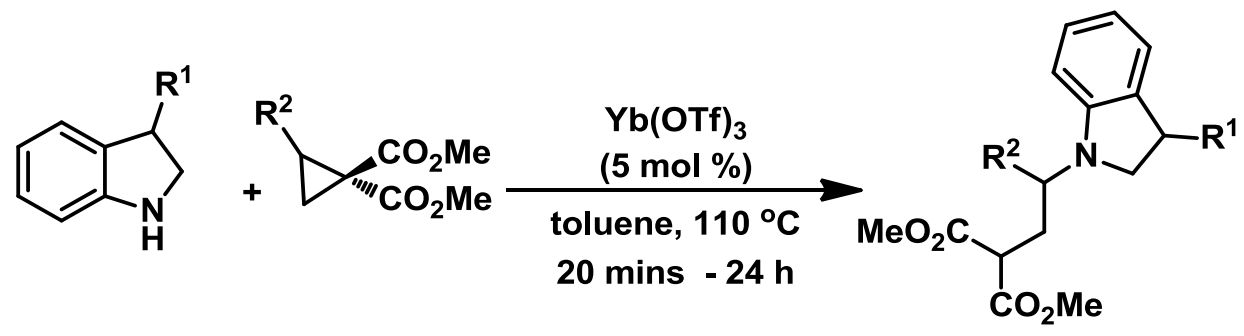

Compounds 5-13 were prepared according to the following procedure. To a solution of indoline 3a-b (1 equiv) in $5 \mathrm{~mL}$ toluene, cyclopropane substrate $4 \mathbf{a}-\mathbf{h}$ (1 equiv), and $\mathrm{Yb}(\mathrm{OTf})_{3}$ (5 mol \%) were added. The reaction mixture was heated to $110^{\circ} \mathrm{C}$ for 20 mins to 24 hours (monitored by TLC). The mixture was then cooled to room temperature and the solvent was removed in vacuo. The crude reaction mixture was pre-absorbed onto silica gel, and purified by column chromatography (EtOAc in Hexanes).

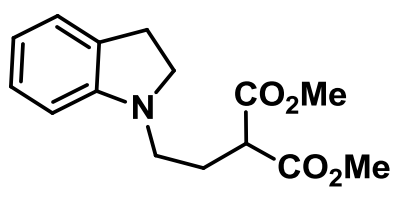

\section{Dimethyl 2-(2-(indolin-1-yl)ethyl)malonate (5).}

Following experimental procedure $\mathrm{B}, \mathrm{N}$-alkylindoline(5) was prepared by dissolving indoline $3 \mathrm{a}$ (75.5 $\mathrm{mg}, 0.633 \mathrm{mmol})$, dimethyl 1,1cyclopropane dicarboxylate $4 \mathrm{a}$ (100 mg, $0.633 \mathrm{mmol})$, and $\mathrm{Yb}(\mathrm{OTf})_{3}(20 \mathrm{mg}, 0.031 \mathrm{mmol})$ in 5 $\mathrm{mL}$ of toluene. The reaction was heated at $110{ }^{\circ} \mathrm{C}$ overnight. Compound 5 (140 mg, 0.505 mmol, $80 \%$ ) was obtained as yellow oil. All spectral data matched that of the literature ${ }^{3}$. ${ }^{1} \mathrm{HNMR}\left(400 \mathrm{MHz}, \mathrm{CDCl}_{3}\right): \delta=7.04-7.12(\mathrm{~m}, 2 \mathrm{H}), 6.68(\mathrm{t}, J=7.3 \mathrm{~Hz}, 1 \mathrm{H}), 6.47(\mathrm{~d}, J=7.6 \mathrm{~Hz}$, $1 \mathrm{H}), 3.73(\mathrm{~s}, 6 \mathrm{H}), 3.58(\mathrm{t}, J=7.0 \mathrm{~Hz}, 1 \mathrm{H}), 3.34(\mathrm{t}, J=8.5 \mathrm{~Hz}, 2 \mathrm{H}), 3.14(\mathrm{t}, J=7.0 \mathrm{~Hz}, 2 \mathrm{H}), 2.97$ (t, $J=8.2 \mathrm{~Hz}, 2 \mathrm{H}$ ), $2.24 \mathrm{ppm}(\mathrm{q}, J=7.0 \mathrm{~Hz}, 2 \mathrm{H}$ ).

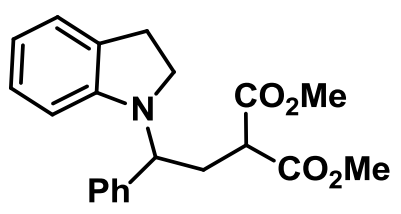

Dimethyl 2-(2-(indolin-1-yl)-2-phenylethyl)malonate (6). Following experimental procedure $\mathrm{B}, \mathrm{N}$-alkylindoline 6 was prepared by dissolving indoline $3 a(50 \mathrm{mg}, 0.419 \mathrm{mmol})$, dimethyl 2-phenylcyclopropane-1,1dicarboxylate 4b (100 mg, $0.419 \mathrm{mmol})$, and $\mathrm{Yb}(\mathrm{OTf})_{3}(13 \mathrm{mg}, 0.021$ $\mathrm{mmol}$ ) in $5 \mathrm{~mL}$ of toluene. The solution was heated at $110^{\circ} \mathrm{C}$ overnight. Compound 6 (123 mg, $0.393 \mathrm{mmol}, 72 \%)$ was as a clear oil. Spectral data matched that of the literature ${ }^{3}$. ${ }^{1} \mathrm{HNMR}$ 
$\left(400 \mathrm{MHz}, \mathrm{CDCl}_{3}\right): \delta=7.27(\mathrm{~m}, 5 \mathrm{H}), 7.05(\mathrm{t}, J=7.6 \mathrm{~Hz}, 1 \mathrm{H}), 7.01(\mathrm{~d}, J=7.0 \mathrm{~Hz}, 1 \mathrm{H}), 6.60$ (t, $J$ $=7.3 \mathrm{~Hz}, 1 \mathrm{H}), 6.56(\mathrm{~d}, J=8.2 \mathrm{~Hz}, 1 \mathrm{H}), 4.80(\mathrm{dd}, J=10.3,5.6 \mathrm{~Hz}, 1 \mathrm{H}), 3.72(\mathrm{~s}, 3 \mathrm{H}), 3.66(\mathrm{~s}$, $3 \mathrm{H}$ ), 3.60 (t, $J=7.0 \mathrm{~Hz}, 1 \mathrm{H}$ ), 3.41 (td, $J=9.1,5.3 \mathrm{~Hz}, 1 \mathrm{H}$ ), 3.07 (q, $J=9.4 \mathrm{~Hz}, 1 \mathrm{H}$ ), $2.84-2.96$ (m, 2H), 2.71 (ddd, $J=14.1,10.0,7.0 \mathrm{~Hz}, \mathrm{H}), 2.53-2.59 \mathrm{ppm}(\mathrm{m}, 1 \mathrm{H})$.

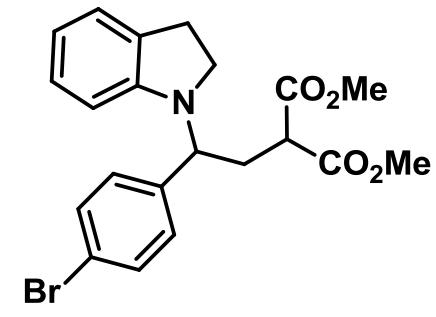

Dimethyl 2-(2-(4-bromophenyl)-2-(indolin-1-yl)ethyl)malonate (7). Following experimental procedure $\mathrm{B}, \mathrm{N}$-alkylindoline 7 was prepared by dissolving indoline $3 \mathrm{a}$ (32 $\mathrm{mg}, 0.272 \mathrm{mmol}$ ), dimethyl 2-(4bromophenyl)cyclopropane-1,1-dicarboxylate $\quad 4 c \quad(85 \mathrm{mg}, \quad 0.272$ $\mathrm{mmol})$, and $\mathrm{Yb}(\mathrm{OTf})_{3}(12 \mathrm{mg}, 0.019 \mathrm{mmol})$ in $5 \mathrm{~mL}$ of toluene. The solution was heated at $110{ }^{\circ} \mathrm{C}$ overnight. Compound 7 (84 mg, $\left.0.194 \mathrm{mmol}, 71 \%\right)$ was obtained as a clear oil. $\mathrm{Rf}=0.53\left(30 \%\right.$ EtOAc in hexanes). ${ }^{1} \mathrm{HNMR}\left(400 \mathrm{MHz}, \mathrm{CDCl}_{3}\right): \delta=7.42$ $(\mathrm{d}, J=8.8 \mathrm{~Hz}, 1 \mathrm{H}), 7.16(\mathrm{~d}, J=8.8 \mathrm{~Hz}, 2 \mathrm{H}), 7.04(\mathrm{dd}, J=8.2,7.6 \mathrm{~Hz}, 1 \mathrm{H}), 7.02(\mathrm{~d}, J=7.0 \mathrm{~Hz}$, $1 \mathrm{H}), 6.64(\mathrm{dd}, J=7.6,7.0 \mathrm{~Hz}, 1 \mathrm{H}), 6.54(\mathrm{~d}, J=7.6 \mathrm{~Hz}, 1 \mathrm{H}), 4.74(\mathrm{dd}, J=9.97,5.87 \mathrm{~Hz}, 1 \mathrm{H})$, $3.71(\mathrm{~s}, 3 \mathrm{H}), 3.66(\mathrm{~s}, 3 \mathrm{H}), 3.56(\mathrm{dd}, J=7.6,7.0 \mathrm{~Hz}, 1 \mathrm{H}), 3.4(\mathrm{~m}, 1 \mathrm{H}), 3.07(\mathrm{~m}, 1 \mathrm{H}), 2.90(\mathrm{~m}$, 2H), 2.67 (ddd, $J=14.1,10.0,7.0 \mathrm{~Hz}, 1 \mathrm{H}), 2.52(\mathrm{~m}, 1 \mathrm{H}) .{ }^{13} \mathrm{CNMR}\left(100 \mathrm{MHz}, \mathrm{CDCl}_{3}\right): \delta=169.7$, 169.6, 150.8, 137.7, 131.6, 129.4, 129.5, 127.3, 124.7, 121.5, 117.4, 106.8, 56.0, 52.6, 52.5, 49.1, 46.2, 30.4, 28.0. FT-IR (thin film, $\mathrm{cm}^{-1}$ ): 2952, 2925, 2853, 1750, 1605, 1487, 1258, 1156, 746. HRMS calc'd for $\mathrm{C}_{21} \mathrm{H}_{22} \mathrm{BrNO}_{4}\left[\mathrm{M}^{+}\right]$: 431.0732; found: 431.0724 .

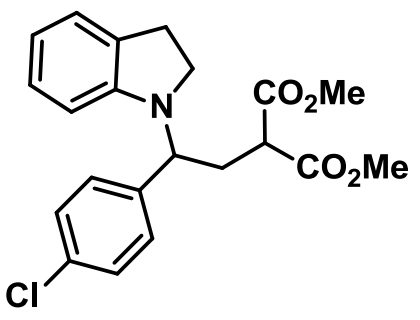

Dimethyl 2-(2-(4-chlorophenyl)-2-(indolin-1-yl)ethyl)malonate (8). Following experimental procedure $\mathrm{B}, \mathrm{N}$-alkylindoline 8 was prepared by dissolving indoline $3 \mathbf{a}(43.5 \mathrm{mg}, 0.373 \mathrm{mmol})$, dimethyl 2-(4chlorophenyl)cyclopropane-1,1-dicarboxylate $\quad$ 4d $\quad(100 \quad \mathrm{mg}$, $0.272 \mathrm{mmol}$ ), and $\mathrm{Yb}(\mathrm{OTf})_{3}(12 \mathrm{mg}, 0.019 \mathrm{mmol})$ in $5 \mathrm{~mL}$ of toluene. The solution was heated at $110{ }^{\circ} \mathrm{C}$ for $3 \mathrm{~h}$. Compound 8 (106 mg, $0.274 \mathrm{mmol}, 73 \%$ ) was obtained as clear oil. $\mathrm{Rf}=0.48\left(30 \%\right.$ EtOAc in hexanes). ${ }^{1} \mathrm{HNMR}\left(400 \mathrm{MHz}, \mathrm{CDCl}_{3}\right): \delta=7.27$ (m, 2H), $7.21(\mathrm{~m}, 2 \mathrm{H}), 7.03(\mathrm{~m}, 2 \mathrm{H}), 6.61(\mathrm{t}, J=7.0 \mathrm{~Hz}, 1 \mathrm{H}), 6.51(\mathrm{~d}, J=7.8 \mathrm{~Hz}, 1 \mathrm{H}), 4.77$ (dd, $J=10.2,5.5 \mathrm{~Hz}, 1 \mathrm{H}) 3.72(\mathrm{~s}, 3 \mathrm{H}), 3.66(\mathrm{~s}, 3 \mathrm{H}), 3.58(\mathrm{dd}, J=7.4,7.0 \mathrm{~Hz}, 1 \mathrm{H}), 3.39(\mathrm{dt}, J=8.8$, $5.5 \mathrm{~Hz}, 1 \mathrm{H}$ ), $3.03(\mathrm{~m}, 1 \mathrm{H}), 2.90(\mathrm{~m}, 2 \mathrm{H}), 2.66$ (ddd, $J=14.1,10.2,7.0 \mathrm{~Hz}, 1 \mathrm{H}), 2.51$ (ddd, $J=$ 14.1, 7.4, $5.5 \mathrm{~Hz}, 1 \mathrm{H}) .{ }^{13} \mathrm{CNMR}\left(100 \mathrm{MHz}, \mathrm{CDCl}_{3}\right): \delta=169.7,169.6,137.2,133.4,129.5$, 129.0, 128.6, 127.3, 124.7, 117.5, 106.8, 56.0, 52.6, 52.5, 49.1, 46.3, 30.4, 28.0. FT-IR (thin 
film, $\left.\mathrm{cm}^{-1}\right): 2951,2847,1733,1489,1258,1156,746$. HRMS calc'd for $\mathrm{C}_{21} \mathrm{H}_{22} \mathrm{CINO}_{4}\left[\mathrm{M}^{+}\right]$: 387.1237; found: 387.1246 .

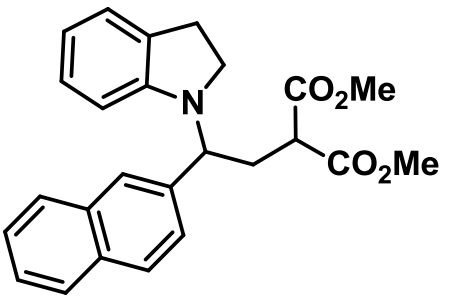

Dimethyl 2-(2-(indolin-1-yl)-2-(naphthalen-2-yl)ethyl)malonate (9). Following experimental procedure $\mathrm{B}, \mathrm{N}$-alkylindoline 9 was prepared by dissolving indoline $3 a(41.5 \mathrm{mg}, 0.352 \mathrm{mmol})$, dimethyl 2(naphthalen-2-yl) cyclopropane-1,1-dicarboxylate 4 e $(100 \mathrm{mg}, 0.352$ $\mathrm{mmol})$, and $\mathrm{Yb}(\mathrm{OTf})_{3}(11 \mathrm{mg}, 0.017 \mathrm{mmol})$ in $5 \mathrm{~mL}$ of toluene. The reaction was heated at $110{ }^{\circ} \mathrm{C}$ overnight. Compound 9 (89 mg, $\left.0.393 \mathrm{mmol}, 63 \%\right)$ was obtained as a white solid. $\mathrm{Mp}=90-93{ }^{\circ} \mathrm{C} . \mathrm{Rf}=0.52$ (30\% EtOAc in hexanes). ${ }^{1} \mathrm{HNMR}$ (400 $\left.\mathrm{MHz}, \mathrm{CDCl}_{3}\right): \delta=8.08(\mathrm{~d}, J=7.0 \mathrm{~Hz}, 1 \mathrm{H}), 7.88(\mathrm{~m}, 1 \mathrm{H}), 7.82(\mathrm{~d}, J=8.2 \mathrm{~Hz}, 1 \mathrm{H}), 7.56(\mathrm{~d}, J=$ $7.04 \mathrm{~Hz}, 1 \mathrm{H}), 7.51-7.47(\mathrm{~m}, 3 \mathrm{H}), 7.14(\mathrm{dd}, J=8.21,7.6 \mathrm{~Hz}, 1 \mathrm{H}), 7.03(\mathrm{~d}, J=7.0 \mathrm{~Hz}, 1 \mathrm{H}), 6.74$ $(\mathrm{d}, J=7.6 \mathrm{~Hz}, 1 \mathrm{H}), 6.65(\mathrm{dd}, J=7.6,7.0 \mathrm{~Hz}, 1 \mathrm{H}), 5.54(\mathrm{t}, J=7.6 \mathrm{~Hz}, 1 \mathrm{H}), 3.74(\mathrm{~s}, 3 \mathrm{H}), 3.67(\mathrm{~s}$, 3H), $3.56(\mathrm{dd}, J=8.2,5.9 \mathrm{~Hz}, 1 \mathrm{H}), 3.44(\mathrm{~m}, 1 \mathrm{H}), 2.99(\mathrm{~m}, 1 \mathrm{H}), 2.91(\mathrm{~m}, 1 \mathrm{H}), 2.79(\mathrm{~m}, 3 \mathrm{H})$. ${ }^{13} \mathrm{CNMR}\left(100 \mathrm{MHz}, \mathrm{CDCl}_{3}\right): \delta=169.8,169.6,150.5,134.3,134.1,132.2,129.5,128.7,128.4$, $127.5,126.5,125.8,124.9,124.5,123.9,123.6,116.9,106.1,52.7,52.5,52.1,48.8,47.1$, 28.3, 28.0. FT-IR (thin film, $\mathrm{cm}^{-1}$ ): 3047, 2951, 2849, 1733, 1605, 1259, 1155. HRMS calc'd for $\mathrm{C}_{25} \mathrm{H}_{25} \mathrm{NO}_{4}\left[\mathrm{M}^{+}\right]:$403.1784; found: 403.1799 .

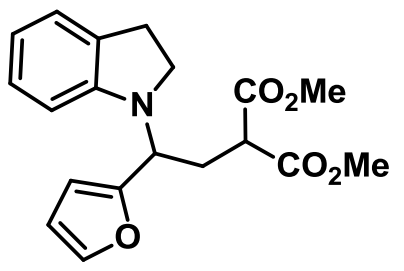

\section{Dimethyl 2-(2-(furan-2-yl)-2-(indolin-1-yl)ethyl)malonate (10).}

Following experimental procedure $\mathrm{B}, \mathrm{N}$-alkylindoline $\mathbf{1 0}$ was prepared

by dissolving indoline $3 \mathrm{a}(53.5 \mathrm{mg}, 0.446 \mathrm{mmol})$, dimethyl 2-(furan-2yl)cyclopropane-1,1,-dicarboxylate $4 \mathrm{f}(100 \mathrm{mg}, 0.446 \mathrm{mmol})$, and $\mathrm{Yb}(\mathrm{OTf})_{3}(14 \mathrm{mg}, 0.022 \mathrm{mmol})$ in $5 \mathrm{~mL}$ of toluene. The reaction was heated at $110{ }^{\circ} \mathrm{C}$ for $4 \mathrm{~h}$. Compound 10 (110 mg $0.320 \mathrm{mmol}, 72 \%)$ was obtained as a light yellow oil. $\mathrm{Rf}=0.62(30 \%$ EtOAc in hexanes). ${ }^{1} \mathrm{HNMR}\left(400 \mathrm{MHz}, \mathrm{CDCl}_{3}\right): \delta=7.29(\mathrm{~d}, J=1.8,1 \mathrm{H}), 7.06(\mathrm{t}, J=7.6 \mathrm{~Hz}$, $1 \mathrm{H}), 7.02(\mathrm{~d}, J=7.0 \mathrm{~Hz}, 1 \mathrm{H}), 6.64(\mathrm{dd}, J=7.6,7.0 \mathrm{~Hz}, 1 \mathrm{H}), 6.56(\mathrm{~d}, J=7.6 \mathrm{~Hz}, 1 \mathrm{H}), 6.26$ (dd, $J=3.5,1.8 \mathrm{~Hz}, 1 \mathrm{H}), 6.17(\mathrm{~d}, J=3.52 \mathrm{~Hz}, 1 \mathrm{H}), 4.80(\mathrm{dd}, J=10.0,6.5 \mathrm{~Hz}, 1 \mathrm{H}), 3.72(\mathrm{~s}, 3 \mathrm{H})$, 3.69 (s, 3H), 3.65 (dd, $J=7.6,7.0 \mathrm{~Hz}, 1 \mathrm{H}$ ), 3.44 (ddd, $J=8.8,8.2,5.3 \mathrm{~Hz}, 1 \mathrm{H}), 3.03(\mathrm{~m}, 1 \mathrm{H})$, $2.90(\mathrm{~m}, 2 \mathrm{H}), 2.58(\mathrm{~m}, 2 \mathrm{H}) .{ }^{13} \mathrm{CNMR}\left(100 \mathrm{MHz}, \mathrm{CDCl}_{3}\right): \delta=169.8,169.5,152.7,150.9,142.0$, 129.7, 127.1, 124.5, 117.8, 109.8, 107.8, 107.4, 52.6, 52.5, 51.2, 48.7, 47.2, 29.4, 28.1. FT-IR 
(thin film, $\mathrm{cm}^{-1}$ ): 3024, 2952, 2847, 1734, 1606, 1486, 1259, 1156, 1013, 745. HRMS calc'd for $\mathrm{C}_{19} \mathrm{H}_{21} \mathrm{NO}_{5}\left[\mathrm{M}^{+}\right]$: 343.1420; found: 343.1421 .

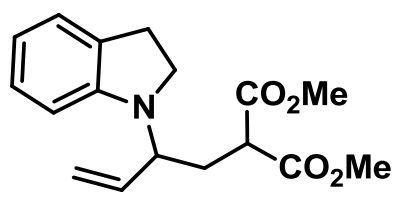

\section{Dimethyl 2-(2-(indolin-1-yl)but-3-en-1-yl)malonate (11).}

Following experimental procedure $\mathrm{B}, \mathrm{N}$-alkylindoline $\mathbf{1 1}$ was prepared by dissolving indoline $3 \mathrm{a}(63.7 \mathrm{mg}, 0.543 \mathrm{mmol})$, dimethyl 2vinylcyclopropane-1,1- dicarboxylate $\mathbf{4 g}(100 \mathrm{mg}, 0.543 \mathrm{mmol})$, and $\mathrm{Yb}(\mathrm{OTf})_{3}(17 \mathrm{mg}, 0.271$ $\mathrm{mmol}$ ) in $5 \mathrm{~mL}$ of toluene. The reaction was heated at $110^{\circ} \mathrm{C}$ overnight. Compound 11 (119 $\mathrm{mg}, 0.393 \mathrm{mmol}, 72 \%)$ was obtained as a clear oil. $\mathrm{Rf}=0.55$ (30\% EtOAc in hexanes). ${ }^{1} \mathrm{HNMR}$ $\left(400 \mathrm{MHz}, \mathrm{CDCl}_{3}\right): \delta=7.06(\mathrm{t}, J=7.82 \mathrm{~Hz}, 2 \mathrm{H}), 6.63(\mathrm{t}, J=7.4 \mathrm{~Hz}, 1 \mathrm{H}), 6.43(\mathrm{~d}, J=7.8 \mathrm{~Hz}$, 1H), 5.78 (ddd, $J=17.2,10.9,6.3 \mathrm{~Hz}, 1 \mathrm{H}$ ), $5.23(\mathrm{~m}, 2 \mathrm{H}), 4.06(\mathrm{dd}, J=7.0,7.4 \mathrm{~Hz}, 1 \mathrm{H}), 3.72$ (s, 3H), $3.69(\mathrm{~s}, 3 \mathrm{H}), 3.62(\mathrm{~m}, 1 \mathrm{H}), 3.38(\mathrm{~m}, 1 \mathrm{H}), 3.31(\mathrm{~m}, 1 \mathrm{H}), 2.93(\mathrm{~m}, 2 \mathrm{H}), 2.32(\mathrm{t}, J=7.03$ $\mathrm{Hz}, 2 \mathrm{H}) .{ }^{13} \mathrm{CNMR}\left(100 \mathrm{MHz}, \mathrm{CDCl}_{3}\right): \delta=169.7,169.3,134.6,130.0,127.2,124.5,121.6$, 119.7, 118.1, 107.8, 55.7, 52.6, 48.7, 46.7, 30.4, 28.3. FT-IR (thin film, $\mathrm{cm}^{-1}$ ): 3081, 3050, 2953, 1739, 1434, 1260, 1210. HRMS calc'd for $\mathrm{C}_{17} \mathrm{H}_{21} \mathrm{NO}_{4}\left[\mathrm{M}^{+}\right]$: 303.1471 ; found: 303.1462.

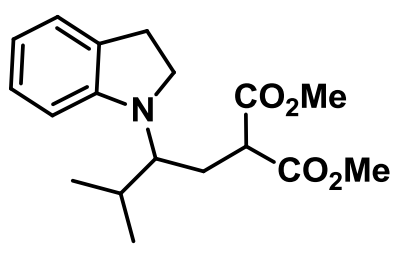

\section{Dimethyl 2-(2-(indolin-1-yl)-3-methylbutyl)malonate (12).}

Following experimental procedure $\mathrm{B}, \mathrm{N}$-alkylindoline 12 was prepared by dissolving indoline $3 a(63.7 \mathrm{mg}, 0.543 \mathrm{mmol})$, dimethyl-2isopropylcyclopropane-1,1-dicarboxylate $4 \mathrm{~h}$ (109 $\mathrm{mg}, 0.543 \mathrm{mmol})$, and $\mathrm{Yb}(\mathrm{OTf})_{3}(17 \mathrm{mg}, 0.027 \mathrm{mmol})$ in $5 \mathrm{~mL}$ of toluene. The reaction was heated at $110{ }^{\circ} \mathrm{C}$ for 24 h. Compound 12 (43 mg, $0.135 \mathrm{mmol}, 24 \%)$ was obtained as a clear oil. $\mathrm{Rf}=0.65$ (30\% EtOAc in hexanes). ${ }^{1} \mathrm{HNMR}\left(400 \mathrm{MHz}, \mathrm{CDCl}_{3}\right): \delta=6.96(\mathrm{~m}, 2 \mathrm{H}), 6.48(\mathrm{t}, J=7.4 \mathrm{~Hz}, 1 \mathrm{H}), 6.22$ (d, $J=7.8 \mathrm{~Hz}, 1 \mathrm{H}), 3.66(\mathrm{~s}, 3 \mathrm{H}), 3.58(\mathrm{~s}, 3 \mathrm{H}), 3.44(\mathrm{~m}, 2 \mathrm{H}), 3.35(\mathrm{~m}, 2 \mathrm{H}), 2.98(\mathrm{~m}, 2 \mathrm{H}), 2.36$ $(\mathrm{m}, 1 \mathrm{H}), 2.06$ (ddd, $J=9.0,6.6,5.1 \mathrm{~Hz}, 1 \mathrm{H}), 1.89(\mathrm{~m}, 1 \mathrm{H}), 1.01(\mathrm{~d}, J=6.6 \mathrm{~Hz}, 3 \mathrm{H}), 0.82(\mathrm{~d}, J=$ $6.6 \mathrm{~Hz}, 3 \mathrm{H}) .{ }^{13} \mathrm{CNMR}(100 \mathrm{MHz}, \mathrm{CDCl} 3): \delta=170.2,169.8,152.0,128.0,127.1,124.4,115.7$, 105.2, 58.6, 52.3, 52.3, 49.2, 30.9, 29.6, 28.0, 20.8, 20.4. FT-IR (thin film, $\mathrm{cm}^{-1}$ ): 3024, 2952, $2847,1734,1606,1486,1259,1156,1013$. HRMS calc'd for $\mathrm{C}_{18} \mathrm{H}_{25} \mathrm{NO}_{4}\left[\mathrm{M}^{+}\right]: 319.1784$; found: 319.1780 . 


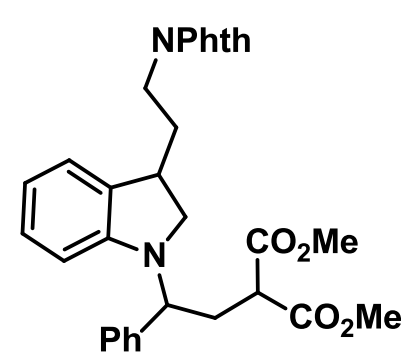

\section{Dimethyl 2-(2-(3-(2-(1,3-dioxoisoindolin-2-yl)ethyl)indolin-1-yl)-2- phenylethyl)malonate (13).}

Following experimental procedure $\mathrm{B}, \mathrm{N}$-alkylindoline 13 was prepared by dissolving phtalamide protected 2-(indolin-3-yl)ethanamine $3 \mathbf{b}^{4}$ (124 $(100 \mathrm{mg}, 0.427 \mathrm{mmol})$, and $\mathrm{Yb}(\mathrm{OTf})_{3}(13 \mathrm{mg}, 0.021 \mathrm{mmol})$. The reaction was heated at $110{ }^{\circ} \mathrm{C}$ for 20 mins. Compound 13 (172 mg, $0.327 \mathrm{mmol}, 77 \%)$ in a 1:1 mixture of diastereomers and as a clear oil. $\mathrm{Rf}=0.55$ (40\% EtOAc in hexanes). ${ }^{1} \mathrm{HNMR}\left(400 \mathrm{MHz}, \mathrm{CDCl}_{3}\right)$ (mixture of diastereomers): $\delta=7.84(\mathrm{~m}, 4 \mathrm{H}), 7.71(\mathrm{~m}, 4 \mathrm{H}), 7.28(\mathrm{~m}, 8 \mathrm{H}), 7.25(\mathrm{~m}, 2 \mathrm{H}), 7.06(\mathrm{~m}, 4 \mathrm{H}), 6.62$ (dd, $J=7.6,7.6 \mathrm{~Hz}, 2 \mathrm{H}), 6.54(\mathrm{~m}, 2 \mathrm{H}), 4.77(\mathrm{~m}, 2 \mathrm{H}), 3.78(\mathrm{~m}, 2 \mathrm{H}), 3.72$ (diastereomer A (s, $3 \mathrm{H})$ ), 3.71 (diastereomer $\mathrm{A}+\mathrm{B}(\mathrm{s}, 6 \mathrm{H}))$, 3.67(diastereomer $\mathrm{B}(\mathrm{s}, 3 \mathrm{H}))$, 3.66-3.55 (m, 5H), $3.33(\mathrm{t}$, $J=8.8 \mathrm{~Hz}, 1 \mathrm{H}$ ), $3.25(\mathrm{dd}, J=8.8,5.3 \mathrm{~Hz}, 1 \mathrm{H}), 3.18(\mathrm{~m}, 1 \mathrm{H}), 3.10(\mathrm{~m}, 1 \mathrm{H}), 2.85$ (t, $J=8.8 \mathrm{~Hz}$, $1 \mathrm{H}), 2.69(\mathrm{~m}, 2 \mathrm{H}), 2.56(\mathrm{~m}, 2 \mathrm{H}), 2.09(\mathrm{~m}, 2 \mathrm{H}), 1.90(\mathrm{~m}, 1 \mathrm{H}), 1.67(\mathrm{~m}, 1 \mathrm{H}),{ }^{13} \mathrm{CNMR}(100 \mathrm{MHz}$, $\mathrm{CDCl}_{3}$ ): (both diastereomers) $\delta=169.7,169.5,168.2,168.1,150.7,150.5,138.5,128.4$, 133.8, 132.1, 132.0, 131.9, 131.8, 128.4, 127.7, 127.7, 127.6, 127.6, 127.5, 127.5, 124.0, 123.6, 123.1, 117.4, 117.2, 106.9, 106.8, 56.4, 56.3, 52.5, 52.4, 52.3, 52.1, 52.0, 49.1, 48.8, 37.8, 37.5, 35.8, 35.7, 33.5, 32.6, 30.5, 30.0. FT-IR (thin film, $\mathrm{cm}^{-1}$ ): 3029, 2951, 1750, 1732, 1712, 1604, 1487, 1397, 1262, 1158, 912, 722. HRMS calc'd for $\mathrm{C}_{31} \mathrm{H}_{30} \mathrm{~N}_{2} \mathrm{O}_{6}\left[\mathrm{M}^{+}\right]: 526.2104$; found 526.2121 .

Experimental Procedure C: $\mathrm{Mn}(\mathrm{OAC})_{3}$ Promoted Cyclization of $\mathrm{N}$-alkylindolines to pyrroloindoles

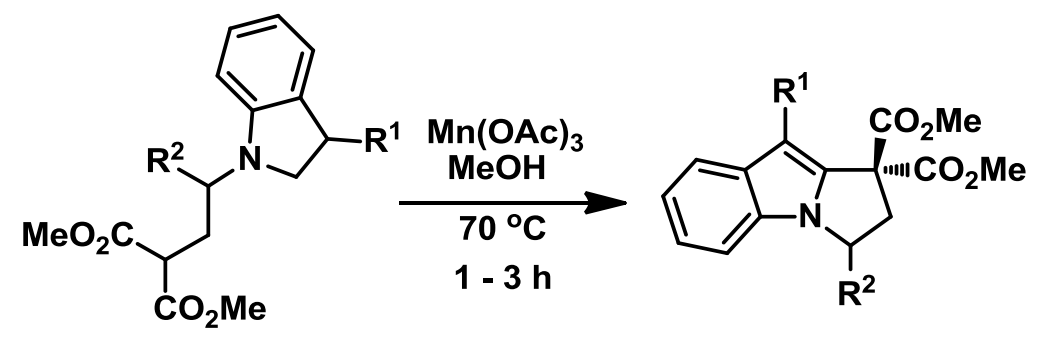

Compounds 14-22 were prepared according to the following procedure. To a solution of $\mathrm{N}$ alkylindoline 5-13 (1 equiv) in $8-15 \mathrm{~mL}$ of glass distilled $\mathrm{MeOH}$ was added $\mathrm{Mn}(\mathrm{OAc})_{3}$ (5 equivs) was added. The reaction mixture was heated at $70{ }^{\circ} \mathrm{C}$ for 30 mins $-24 \mathrm{~h}$ (monitored by TLC). The crude reaction mixture was concentrated and the resulting brown solid was partitioned between EtOAc and brine. The organic layer was removed and the aqueous layer 
was extracted with EtOAc ( $3 x s)$. The organic layers were combined, washed with water ( $3 x s)$, once with brine, dried over $\mathrm{MgSO}_{4}$, and filtered. The filtrate was concentrated; the residue was pre-absorbed onto silica gel and purified by column chromatography (EtOAc in Hexanes).

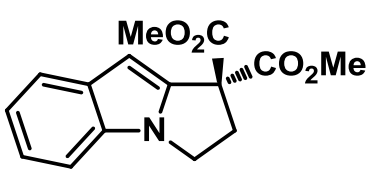

Dimethyl 2,3-dihydro-1H-pyrrolo[1,2-a]indole-1,1-dicarboxylate (14). Following experimental procedure $\mathrm{C}$, pyrroloindole 14 was prepared by dissolving $\mathrm{N}$-alkylindoline 5 (120 $\mathrm{mg}, 0.433 \mathrm{mmol})$ and $\mathrm{Mn}(\mathrm{OAc})_{3}(482$ $\mathrm{mg}, 1.80 \mathrm{mmol})$ in $5 \mathrm{~mL}$ of $\mathrm{MeOH}$. Compound $14(97 \mathrm{mg}, 0.355 \mathrm{mmol}, 82 \%)$ was obtained as a white solid. Spectral data for this compound matched that of the literature ${ }^{3}$. ${ }^{1} \mathrm{HNMR}(400 \mathrm{MHz}$, $\left.\mathrm{CDCl}_{3}\right): \delta=7.61(\mathrm{~d}, J=7.6 \mathrm{~Hz}, 1 \mathrm{H}), 7.26-7.29(\mathrm{~m}, 2 \mathrm{H}), 7.19(\mathrm{t}, J=7.6,1.2 \mathrm{~Hz}, 1 \mathrm{H}), 7.09-7.13$ (m, 1H), $6.55(\mathrm{~s}, 1 \mathrm{H}), 4.22(\mathrm{t}, J=6.7 \mathrm{~Hz}, 2 \mathrm{H}), 3.81(\mathrm{~s}, 6 \mathrm{H}), 3.20$ (t, $J=7.0 \mathrm{~Hz}, 2 \mathrm{H})$.

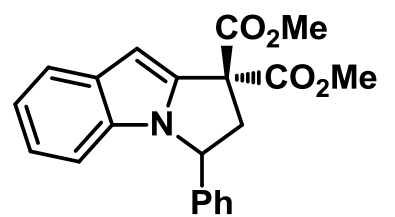

Dimethyl 3-phenyl-2,3-dihydro-1H-pyrrolo[1,2-a]indole-1,1dicarboxylate (15).

Following experimental procedure $\mathrm{C}$, pyrroloindole $\mathbf{1 5}$ was prepared by dissolving N-alkylindoline 6 (50 mg, $0.142 \mathrm{mmol}$ ), $\mathrm{Mn}(\mathrm{OAc})_{3}(151 \mathrm{mg}, 0.566 \mathrm{mmol}$ ) in $5 \mathrm{~mL}$ of $\mathrm{MeOH}$. The reaction was heated at $70{ }^{\circ} \mathrm{C}$ overnight. Compound 15 (42 mg, $\left.0.121 \mathrm{mmol}, 86 \%\right)$ was obtained as a white solid. Rf $=0.50$ (30\% EtOAc in hexanes). Spectral data for this compound matched that of the literature ${ }^{3} .{ }^{1} \mathrm{HNMR}\left(400 \mathrm{MHz}, \mathrm{CDCl}_{3}\right): \delta=7.53(\mathrm{~d}, J=8.2 \mathrm{~Hz}$, $1 \mathrm{H}), 7.24-7.30(\mathrm{~m}, 3 \mathrm{H}), 7.15-7.20(\mathrm{~m}, 2 \mathrm{H}), 6.97(\mathrm{t}, J=7.3 \mathrm{~Hz}, 1 \mathrm{H}), 6.87(\mathrm{t}, J=7.6 \mathrm{~Hz}, 1 \mathrm{H})$, $6.55(\mathrm{~d}, J=8.2 \mathrm{~Hz}, 1 \mathrm{H}), 6.53(\mathrm{~s}, 1 \mathrm{H}), 5.44(\mathrm{t}, J=7.6 \mathrm{~Hz}, 1 \mathrm{H}), 3.73(\mathrm{~s}, 3 \mathrm{H}), 3.71(\mathrm{~s}, 3 \mathrm{H}), 3.58$ (dd, $J=13.8,7.3 \mathrm{~Hz}, 1 \mathrm{H}$ ), $2.94(\mathrm{dd}, J=13.5,7.6 \mathrm{~Hz}, 1 \mathrm{H}$ ).

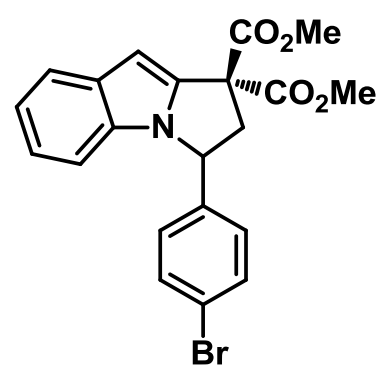

Dimethyl 3-(4-bromophenyl)-2,3-dihydro-1H-pyrrolo[1,2-a]indole-1,1dicarboxylate (16).

Following experimental procedure $\mathrm{C}$, pyrroloindole 16 was prepared by dissolving $\mathrm{N}$-alkylindoline 7 (75 mg, $0.175 \mathrm{mmol})$ and $\mathrm{Mn}(\mathrm{OAc})_{3}(234 \mathrm{mg}$, $0.875 \mathrm{mmol}$ ) in $5 \mathrm{~mL}$ of $\mathrm{MeOH}$. The reaction was heated at $70{ }^{\circ} \mathrm{C}$ overnight. Compound 16 (63 mg, $0.147 \mathrm{mmol}, 84 \%$ ) was obtained as a clear oil. $\mathrm{Rf}=0.55\left(30 \%\right.$ EtOAc in hexanes). ${ }^{1} \mathrm{HNMR}\left(400 \mathrm{MHz}, \mathrm{CDCl}_{3}\right): \delta=7.61$ (d, $J=7.0$ $\mathrm{Hz}, 1 \mathrm{H}), 7.49,(\mathrm{~d}, J=8.6 \mathrm{~Hz}, 2 \mathrm{H}), 7.12(\mathrm{~d}, J=8.6 \mathrm{~Hz}, 2 \mathrm{H}), 7.07(\mathrm{dd}, J=7.4,7.0 \mathrm{~Hz}, 1 \mathrm{H}), 6.99$ 
(dd, $J=8.2,7.0 \mathrm{~Hz}, 1 \mathrm{H}), 6.64(\mathrm{~d}, J=8.2 \mathrm{~Hz}, 1 \mathrm{H}), 6.61(\mathrm{~s}, 1 \mathrm{H}), 5.49(\mathrm{t}, J=7.4 \mathrm{~Hz}, 1 \mathrm{H}), 3.82(\mathrm{~s}$, $3 \mathrm{H}), 3.79(\mathrm{~s}, 3 \mathrm{H}), 3.66(\mathrm{dd}, J=13.7,7.4 \mathrm{~Hz}, 1 \mathrm{H}), 2.96(\mathrm{dd}, J=13.7,7.4 \mathrm{~Hz}, 1 \mathrm{H}) .{ }^{13} \mathrm{CNMR}(100$ $\left.\mathrm{MHz}_{\mathrm{CDCl}}\right): \delta=169.2,169.1,139.2,138.5,132.9,132.3,132.1,128.4,122.3,121.7,121.4$, 120.0, 110.5, 97.2, 59.5, 58.5, 53.5, 53.4, 47.2. FT-IR (thin film, $\mathrm{cm}^{-1}$ ): 2953, 2923, 2850, 1738 , 1257, 747. HRMS calc'd for $\mathrm{C}_{21} \mathrm{H}_{18} \mathrm{BrNO}_{4}\left[\mathrm{M}^{+}\right]$: 427.0419; found: 427.0410 .

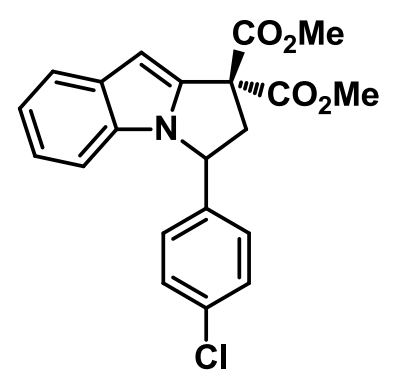

Dimethyl 3-(4-chlorophenyl)-2,3-dihydro-1H-pyrrolo[1,2-a]indole-1,1dicarboxylate (17).

Following experimental procedure $\mathrm{C}$, pyrroloindole $\mathbf{1 7}$ was prepared by dissolving $\mathrm{N}$-alkylindoline 8 (48 $\mathrm{mg}, 0.123 \mathrm{mmol})$ and $\mathrm{Mn}(\mathrm{OAc})_{3}(164$ $\mathrm{mg}, 0.614 \mathrm{mmol}$ ) in $3 \mathrm{~mL}$ of $\mathrm{MeOH}$. The reaction was heated at $70{ }^{\circ} \mathrm{C}$ overnight. Compound 17 (29 mg, $0.077 \mathrm{mmol}, 63 \%$ ) was obtained as a clear oil. $\mathrm{Rf}=0.60$ (30\% EtOAc in hexanes). ${ }^{1} \mathrm{HNMR}\left(400 \mathrm{MHz}, \mathrm{CDCl}_{3}\right): \delta=7.61$ (d, $J=8.2$ $\mathrm{Hz}, 1 \mathrm{H}), 7.34(\mathrm{~d}, J=7.6 \mathrm{~Hz}, 2 \mathrm{H}), 7.19(\mathrm{~d}, J=7.6 \mathrm{~Hz}, 2 \mathrm{H}), 7.07$ (dd, $J=8.2,7.0 \mathrm{~Hz}, 1 \mathrm{H}), 6.98$ (dd, $J=8.2,7.0 \mathrm{~Hz}, 1 \mathrm{H}), 6.64(\mathrm{~d}, J=8.2 \mathrm{~Hz}, 1 \mathrm{H}), 6.61(\mathrm{~s}, 1 \mathrm{H}) 5.50(\mathrm{dd}, J=7.6,7.0 \mathrm{~Hz}, 1 \mathrm{H}$ ), $3.82(\mathrm{~s}, 3 \mathrm{H}), 3.80(\mathrm{~s}, 3 \mathrm{H}), 3.66(\mathrm{dd}, J=13.5,7.0 \mathrm{~Hz}, 1 \mathrm{H}), 2.97$ (dd, $J=13.8,7.6 \mathrm{~Hz}, 1 \mathrm{H}$ ). ${ }^{13} \mathrm{CNMR}\left(100 \mathrm{MHz}, \mathrm{CDCl}_{3}\right): \delta=169.2,139.2,138.0,134.2,132.9,132.4,129.2,128.1,121.7$, 121.4, 120.0, 110.5, 105.4, 97.2, 59.5, 58.6, 53.5, 53.4, 47.3. FT-IR (thin film, $\mathrm{cm}^{-1}$ ): 2953, 2924, 2852, 1739, 1274, 1258, 746. HRMS calc'd for $\mathrm{C}_{21} \mathrm{H}_{18} \mathrm{CINO}_{4}\left[\mathrm{M}^{+}\right]: 383.0924$; found: 383.0931 .

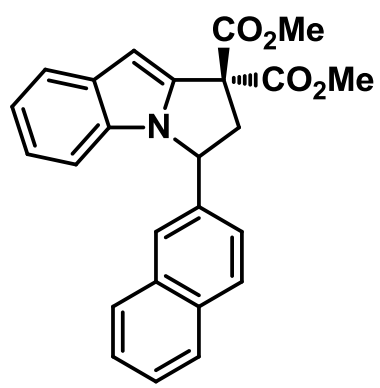

Dimethyl 3-(naphthalen-2-yl)-2,3-dihydro-1H-pyrrolo[1,2-a]indole-1,1dicarboxylate (18). Following experimental procedure $\mathrm{C}$, pyrroloindole 18 was prepared by dissolving $N$-alkylindoline $9(68 \mathrm{mg}, 0.168 \mathrm{mmol})$ and $\mathrm{Mn}(\mathrm{OAc})_{3}(225 \mathrm{mg}, 0.841 \mathrm{mmol})$ in $5 \mathrm{~mL}$ of $\mathrm{MeOH}$. The reaction was heated at $70{ }^{\circ} \mathrm{C}$ overnight. Compound $18(41 \mathrm{mg}, 0.103 \mathrm{mmol}, 61 \%)$ was obtained as a white solid. $\mathrm{Rf}=0.56$ (30\% EtOAc in hexanes). ${ }^{1} \mathrm{HNMR}$ $\left(400 \mathrm{MHz}, \mathrm{CDCl}_{3}\right.$ ): $\delta=8.13$ (broad $\mathrm{m}, 1 \mathrm{H}$ ), 7.94 (broad $\left.\mathrm{m}, 1 \mathrm{H}\right), 7.82$ (broad $\left.\mathrm{m}, 1 \mathrm{H}\right), 7.67-7.52$ (broad m, 4H), 7.31 (broad m, 1H), 7.08 (broad m, 1H), 7.00 (broad m, 1H), 6.91 (broad m, $1 \mathrm{H}), 6.80$ (broad $\mathrm{m}, 1 \mathrm{H}), 6.69(\mathrm{~s}, 1 \mathrm{H}), 6.37$ (broad $\mathrm{m}, 1 \mathrm{H}), 4.02$, broad $\mathrm{m}, 1 \mathrm{H}$ ), 3.85, s, 3H), 3.66 (broad s, 3H), 3.06 (broad m, 1H). ${ }^{13} \mathrm{CNMR}\left(100 \mathrm{MHz}, \mathrm{CDCl}_{3}\right.$ ): $\delta=169.4,169.2,135.0$, 133.9, 132.9, 132.6, 130.3, 129.2, 128.3, 126.7, 125.9, 125.4, 122.8, 122.5, 121.6, 121.4, 
120.0, 111.1, 97.3, 58.6, 56.0, 53.6, 53.2, 46.3, 29.7. FT-IR (thin film, $\mathrm{cm}^{-1}$ ): 3052, 2953, 1739, 1454, 1251, 748. HRMS calc'd for $\mathrm{C}_{25} \mathrm{H}_{21} \mathrm{NO}_{4}\left[\mathrm{M}^{+}\right]$: 399.1471; found: 399.1461.

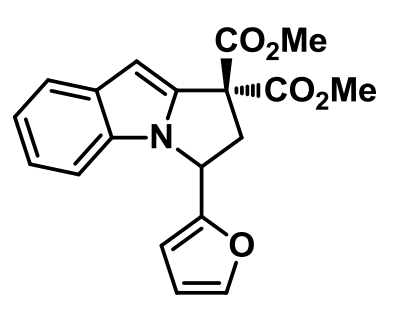

Dimethyl 3-(furan-2-yl)-2,3-dihydro-1H-pyrrolo[1,2-a]indole-1,1dicarboxylate (19).

Following experimental procedure $\mathrm{C}$, pyrroloindole 19 was prepared by dissolving $N$-alkylindoline 10 (150 mg, $0.438 \mathrm{mmol}$ ) and $\mathrm{Mn}(\mathrm{OAc}) 3 . \mathrm{H} 2 \mathrm{O}$ (587 mg, $2.19 \mathrm{mmol})$ in $5 \mathrm{~mL}$ of $\mathrm{MeOH}$. The reaction was heated at $70{ }^{\circ} \mathrm{C}$ overnight. Compound 19 (111 mg, $0.327 \mathrm{mmol}, 75 \%)$ was obtained as a white solid. $\mathrm{Mp}=$ $109-112{ }^{\circ} \mathrm{C} . \mathrm{Rf}=0.41\left(30 \%\right.$ EtOAc in hexanes). ${ }^{1} \mathrm{HNMR}\left(400 \mathrm{MHz}, \mathrm{CDCl}_{3}\right): \delta=7.59(\mathrm{~m}, 1 \mathrm{H})$, $7.41(\mathrm{~m}, 1 \mathrm{H}), 7.06(\mathrm{~m}, 2 \mathrm{H}), 6.97(\mathrm{~m}, 1 \mathrm{H}), 6.57(\mathrm{~s}, 1 \mathrm{H}), 6.39(\mathrm{~m}, 2 \mathrm{H}), 5.64(\mathrm{dd}, J=7.4,7.0 \mathrm{~Hz}$, $1 \mathrm{H}$ ), 3.84 (s, 3H), 3.79 (s, 3H), 3.54 (dd, $J=13.7,7.4 \mathrm{~Hz}, 1 \mathrm{H}$ ), 3.33 (dd, $J=13.4,7.0 \mathrm{~Hz}, 1 \mathrm{H}$ ). ${ }^{13} \mathrm{CNMR}\left(100 \mathrm{MHz}, \mathrm{CDCl}_{3}\right): \delta=169.3,169.1,151.09,143.02,138.4,132.7,132.6,121.7$, 121.3, 119.9, 110.4, 110.0, 108.7, 97.0, 58.3, 53.5, 53.2, 43.10. FT-IR (thin film, $\mathrm{cm}^{-1}$ ): 2953, 1740, 1453, 1270, 1220, 746. HRMS calc'd for $\mathrm{C}_{19} \mathrm{H}_{17} \mathrm{NO}_{5}\left[\mathrm{M}^{+}\right]: 339.1107$; found: 339.1116 .

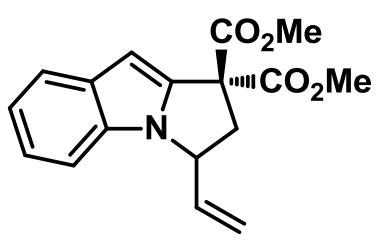

\section{Dimethyl-3-vinyl-2,3-dihydro-1H-pyrrolo[1,2-a]indole-1,1- dicarboxylate (20).}

Following experimental procedure $\mathrm{C}$, pyrroloindole $\mathbf{2 0}$ was prepared by dissolving $\mathrm{N}$-alkylindoline 11 (77 mg, $0.253 \mathrm{mmol})$ and $\mathrm{Mn}(\mathrm{OAc})_{3}$ (339 $\mathrm{mg}, 1.2 \mathrm{mmol}$ ) in $5 \mathrm{~mL}$ of $\mathrm{MeOH}$. The reaction was heated at $70^{\circ} \mathrm{C}$ overnight. Compound 20 (69 mg, $0.231 \mathrm{mmol}, 91 \%)$ was obtained as a white solid. $\mathrm{Mp}=77-79{ }^{\circ} \mathrm{C} . \mathrm{Rf}=0.60(30 \%$ EtOAc in hexanes). ${ }^{1} \mathrm{HNMR}\left(400 \mathrm{MHz}, \mathrm{CDCl}_{3}\right): \delta=7.60(\mathrm{~d}, J=7.6 \mathrm{~Hz}, 1 \mathrm{H}), 7.34(\mathrm{~d}, J=8.21$ $\mathrm{Hz}, 1 \mathrm{H}), 7.12-7.08(\mathrm{~m}, 2 \mathrm{H}), 6.53(\mathrm{~s}, 1 \mathrm{H}), 5.99$ (ddd, $J=17.0,10.0,8.2 \mathrm{~Hz}, 1 \mathrm{H}), 5.50(\mathrm{~d}, J=$ $17.0 \mathrm{~Hz}, 1 \mathrm{H}$ ), $5.37(\mathrm{~d}, J=10.6 \mathrm{~Hz}, 1 \mathrm{H}), 5.00(\mathrm{~m}, 1 \mathrm{H}), 3.83(\mathrm{~s}, 3 \mathrm{H}), 3.77(\mathrm{~s}, 3 \mathrm{H}), 3.40$ (dd, $J=$ 13.5, $7.6 \mathrm{~Hz}, 1 \mathrm{H}), 2.96(\mathrm{dd}, J=13.5,6.5 \mathrm{~Hz}, 1 \mathrm{H}) .{ }^{13} \mathrm{CNMR}\left(100 \mathrm{MHz}, \mathrm{CDCl}_{3}\right): \delta=169.3$, 138.6, 136.8, 132.9, 132.7, 121.5, 121.3, 119.8, 118.8, 110.3, 96.8, 59.1, 58.4, 53.4, 53.3, 43.9, (41.9 artifact from NMR), 29.7. FT-IR (thin film, $\mathrm{cm}^{-1}$ ): 2953, 2852, 1739, 1454, 1273, 746. HRMS calc'd for $\mathrm{C}_{17} \mathrm{H}_{17} \mathrm{NO}_{4}\left[\mathrm{M}^{+}\right]$: 299.1158; found: 299.1148 . 


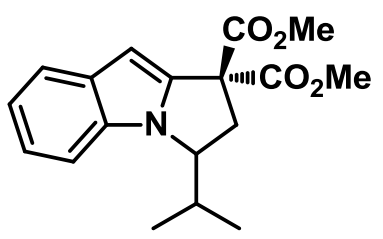

\section{Dimethyl 3-isopropyl-2,3-dihydro-1H-pyrrolo[1,2-a]indole-1,1-} dicarboxylate (21).

Following experimental procedure $\mathrm{C}$, pyrroloindole $\mathbf{2 1}$ was prepared by dissolving $\mathrm{N}$-alkylindoline 12 (22 $\mathrm{mg}, 0.069 \mathrm{mmol})$ and $\mathrm{Mn}(\mathrm{OAc})_{3}(93$ $\mathrm{mg}, 0.345 \mathrm{mmol}$ ) in $2 \mathrm{~mL}$ of $\mathrm{MeOH}$. The reaction was at reflux for $6 \mathrm{~h}$ (monitored by TLC). Compound 21 (13 mg, $0.042 \mathrm{mmol}, 60 \%$ ) was obtained as a clear oil. $\mathrm{Rf}=0.67$ (30\% EtOAc in hexanes). ${ }^{1} \mathrm{HNMR}\left(400 \mathrm{MHz}, \mathrm{CDCl}_{3}\right): \delta=7.63$ (broad dd, $\left.J=8.2,1.2 \mathrm{~Hz}, 1 \mathrm{H}\right), 7.39$ (dd, $J=$ 7.4, $0.8 \mathrm{~Hz}, 1 \mathrm{H}$ ), 7.17 (ddd, $J=8.2,7.0,1.2 \mathrm{~Hz}, 1 \mathrm{H}$ ), 7.11 (ddd, $J=7.8,7.03,0.8 \mathrm{~Hz}, 1 \mathrm{H}$ ), 6.52 (s, $1 \mathrm{H}), 4.60(1 \mathrm{H}$, ddd, $J=7.8,6.6,4.7 \mathrm{~Hz}, 1 \mathrm{H}), 3.87(\mathrm{~s}, 2 \mathrm{H}), 3.77(\mathrm{~s}, 3 \mathrm{H}), 3.21$ (dd, $J=14.1$, $7.8 \mathrm{~Hz}, 1 \mathrm{H}$ ), $2.98(\mathrm{dd}, J=14.1,6.6 \mathrm{~Hz}, 1 \mathrm{H}), 2.82(\mathrm{dqq}, J=7.0,6.6,4.7 \mathrm{~Hz}, 1 \mathrm{H}), 1.11(\mathrm{~d}, J=$ $7.0 \mathrm{~Hz}, 3 \mathrm{H}), 0.71$ (d, $J=6.6 \mathrm{~Hz}, 3 \mathrm{H}) .{ }^{13} \mathrm{CNMR}\left(100 \mathrm{MHz}, \mathrm{CDCl}_{3}\right): \delta=169.7,139.4,132.9$, 132.5, 121.4, 121.3, 119.7, 110.3, 96.4, 61.4, 53.4, 53.3, 36.7, 29.6, 19.3, 14.5. FT-IR (thin film, $\left.\mathrm{cm}^{-1}\right)$ : 2956, 2920, 2849, 1739, 1248, 1106, 746. HRMS calc'd for $\mathrm{C}_{18} \mathrm{H}_{21} \mathrm{NO}_{4}\left[\mathrm{M}^{+}\right]$: 315.1471; found: 315.1469 .

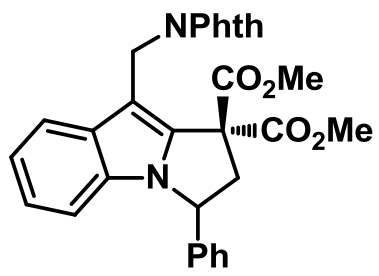

Dimethyl 9-(2-(1,3-dioxoisoindolin-2-yl)ethyl)-3-phenyl-2,3-dihydro1H-pyrrolo[1,2-a]indole-1,1-dicarboxylate (22).

Following experimental procedure $\mathrm{C}$, pyrroloindole 22 was prepared by dissolving $\mathrm{N}$-alkylindoline 13 (165 mg, $0.313 \mathrm{mmol}$ ) and $\mathrm{Mn}(\mathrm{OAc})_{3}$ (420 $\mathrm{mg}, 1.56 \mathrm{mmol}$ ) in $4 \mathrm{~mL}$ of $\mathrm{MeOH}$. The reaction was heated at $70{ }^{\circ} \mathrm{C}$ for 30 mins (monitored by TLC). Compound 22 (151 mg, $0.289 \mathrm{mmol}, 92 \%)$ was obtained as a yellow solid. $\mathrm{Mp}=156-$ $158{ }^{\circ} \mathrm{C} . \mathrm{Rf}=0.62\left(40 \%\right.$ EtOAc in hexanes). ${ }^{1} \mathrm{HNMR}\left(400 \mathrm{MHz}, \mathrm{CDCl}_{3}\right): \delta=7.92(\mathrm{~d}, \mathrm{~J}=8.2 \mathrm{~Hz}$, $1 \mathrm{H}), 7.88(\mathrm{~m}, 2 \mathrm{H}), 7.73(\mathrm{~m}, 2 \mathrm{H}), 7.37(\mathrm{~m}, 3 \mathrm{H}), 7.30(\mathrm{~m}, 2 \mathrm{H}), 7.11$ (apparent t, $J=7.0 \mathrm{~Hz}, 1 \mathrm{H}$ ), 6.98 (apparent t, $J=7.0 \mathrm{~Hz}, 1 \mathrm{H}$ ), $6.61(\mathrm{~d}, J=8.2 \mathrm{~Hz}, 1 \mathrm{H}$ ), $5.49(\mathrm{t}, J=7.4 \mathrm{~Hz}, 1 \mathrm{H}), 4.00(\mathrm{~m}$, 2H), $3.91(\mathrm{~s}, 6 \mathrm{H}), 3.77(\mathrm{dd}, J=13.3,7.0 \mathrm{~Hz}, 1 \mathrm{H}), 3.25(\mathrm{~m}, 2 \mathrm{H}), 2.98(\mathrm{dd}, J=13.3,7.8 \mathrm{~Hz}, 1 \mathrm{H}$ ). ${ }^{13} \mathrm{CNMR}\left(100 \mathrm{MHz}, \mathrm{CDCl}_{3}\right): \delta=169.5,169.3,168.3,139.3,136.0,133.8,132.6,132.3,132.2$, 128.9, 128.3, 126.8, 123.1, 121.8, 119.8, 119.7, 110.6, 106.5, 59.9, 58.7, 53.6, 53.5, 48.3, 37.7, 24.0. FT-IR (thin film, $\mathrm{cm}^{-1}$ ): 3055, 3031, 2953, 1737, 1712, 1397, 1363, 1246, 911, 716. HRMS calc'd for $\mathrm{C}_{31} \mathrm{H}_{26} \mathrm{~N}_{2} \mathrm{O}_{6}\left[\mathrm{M}^{+}\right]$: 522.1791 ; found: 522.1793 . 
Experimental Procedure D: Indoline Ring Opening at Quaternary Donor Sites of Cyclopropanes
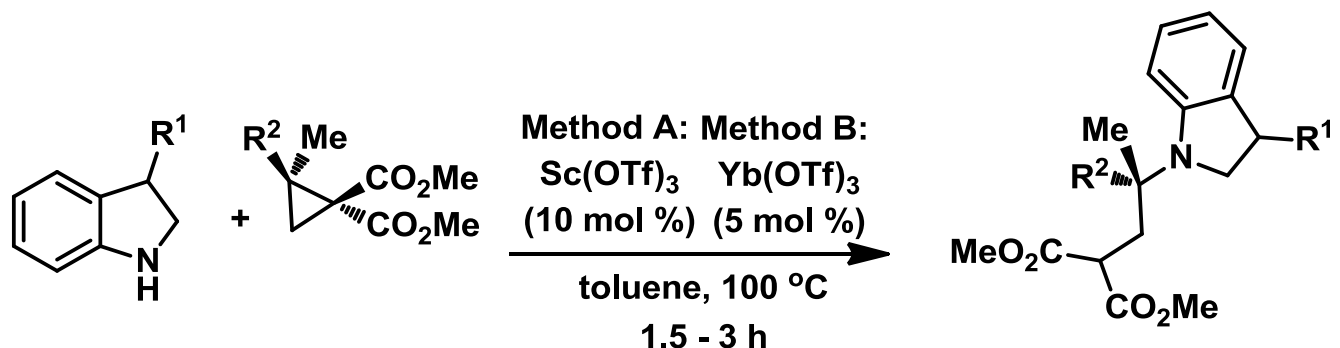

Compounds 23-29 were prepared according to the following procedure. To a solution of the cyclopropane $4 \mathbf{i}-\mathbf{m}$ ( 1 equiv) in $5-10 \mathrm{~mL}$ of toluene, indoline $3 \mathbf{a}, 3 \mathbf{c}-\mathbf{d}$ (2 equiv) and $\mathrm{Sc}(\mathrm{OTf})_{3}$ catalyst (10 mol \%) (Method A) or $\mathrm{Yb}(\mathrm{OTf})_{3}$ catalyst (5 mol \%) (Method B) were added. The reaction mixture was heated at $90{ }^{\circ} \mathrm{C}$ for $1.5-3$ hours (monitored by TLC). The mixture was then cooled to room temperature and diluted with $1 \mathrm{M} \mathrm{HCl}(20 \mathrm{~mL})$. The organic layer was collected and the aqueous layer was extracted with EtOAc $(2 \times 15 \mathrm{~mL})$. The organic layers were combined, washed once with brine, dried over $\mathrm{MgSO}_{4}$, and filtered. The filtrate was concentrated and purified by column chromatography (EtOAc in Hexanes).

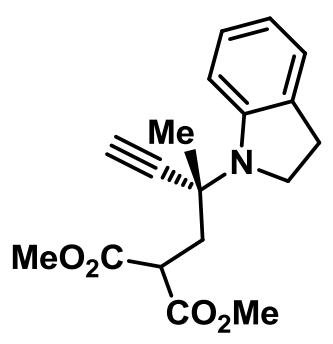

Dimethyl 2-(2-(indolin-1-yl)-2-methylbut-3-ynyl)malonate (23). Following experimental procedure D (Method A), $\mathrm{N}$-alkylindoline $\mathbf{2 3}$ was prepared by dissolving cyclopropane $4 \mathbf{i}(200 \mathrm{mg}, 1.02 \mathrm{mmol}), \mathrm{Sc}(\mathrm{OTf})_{3}(50 \mathrm{mg}, 0.102$ $\mathrm{mmol})$, and indoline $3 \mathrm{a}(243 \mathrm{mg}, 2.04 \mathrm{mmol})$ in $8 \mathrm{~mL}$ of toluene. The reaction was hated at $100{ }^{\circ} \mathrm{C}$ for $2 \mathrm{~h}$. Compound $23(285 \mathrm{~g}, 0.903 \mathrm{mmol}$, $88 \%$ ) was obtained as a brown oil. $\mathrm{Rf}=0.47$ (30\% EtOAc in hexanes).

Following experimental procedure D (Method B), N-alkylindoline 23 was prepared by dissolving cyclopropane $4 \mathbf{i}$ (169 mg, $0.861 \mathrm{mmol}), \mathrm{Yb}(\mathrm{OTf})_{3}(27 \mathrm{mg}, 0.043 \mathrm{mmol})$, and indoline 3a $(204 \mathrm{mg}, 1.72 \mathrm{mmol})$ in $5 \mathrm{~mL}$ of toluene. The reaction was hated at $100{ }^{\circ} \mathrm{C}$ for $2 \mathrm{~h}$. Compound 23 (209 mg, $0.680 \mathrm{mmol}, 77 \%$ ) was obtained as a brown oil.

${ }^{1} \mathrm{HNMR}\left(400 \mathrm{MHz}, \mathrm{CDCl}_{3}\right): \delta=7.10(\mathrm{~m}, 3 \mathrm{H}), 6.70(\mathrm{td}, J=7.4,1.2 \mathrm{~Hz}, 1 \mathrm{H}), 3.86(\mathrm{t}, J=5.9 \mathrm{~Hz}$, $1 \mathrm{H}), 3.65(\mathrm{~s}, 3 \mathrm{H}), 3.63(\mathrm{~s}, 3 \mathrm{H}), 3.43(\mathrm{td}, J=7.8,1.9 \mathrm{~Hz}, 1 \mathrm{H}), 2.88(\mathrm{t}, J=7.8 \mathrm{~Hz}, 2 \mathrm{H}), 2.73(\mathrm{dd}$, $J=14.4,5.8 \mathrm{~Hz}, 1 \mathrm{H}), 2.55$ (dd, $J=14.4,7.0 \mathrm{~Hz}, 1 \mathrm{H}), 2.44(\mathrm{~s}, 1 \mathrm{H}), 1.55(\mathrm{~s}, 3 \mathrm{H}) .{ }^{13} \mathrm{CNMR}(100$ $\left.\mathrm{MHz}, \mathrm{CDCl}_{3}\right): \delta=169.7,169.6,148.9,126.6,124.3,118.5,111.6,84.9,73.3,54.1,52.4,49.9$, 
48.5, 37.9, 27.9, 23.4. FT-IR (thin film, $\mathrm{cm}^{-1}$ ): 3272, 2990, 2952, 2844, 2112, 1732, 1605, 1483, 1331, 1263, 1155, 750, 655. HRMS calc'd for $\mathrm{C}_{18} \mathrm{H}_{21} \mathrm{NO}_{4}\left[\mathrm{M}^{+}\right]: 315.1471$; found: 315.1474 .

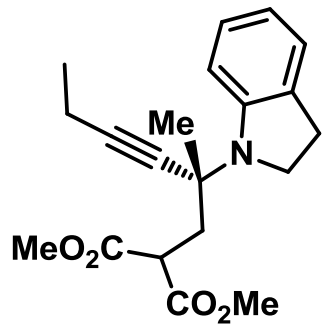

Dimethyl 2-(2-(indolin-1-yl)-2-methylhex-3-ynyl)malonate (24).

Following experimental procedure D (Method A), N-alkylindoline 24 was prepared by dissolving cyclopropane $4 \mathbf{j}$ (332 $\mathrm{mg}, 1.48 \mathrm{mmol}), \mathrm{Sc}(\mathrm{OTf})_{3}(73$ $\mathrm{mg}, 0.148 \mathrm{mmol})$, and indoline $3 \mathbf{a}(353 \mathrm{mg}, 2.96 \mathrm{mmol})$ in $10 \mathrm{~mL}$ toluene. The reaction was hated at $100{ }^{\circ} \mathrm{C}$ for $2 \mathrm{~h}$. Compound 24 was obtained as a yellow oil (365 mg, $1.06 \mathrm{mmol}, 72 \%) . \mathrm{Rf}=0.50$ (30\% EtOAc in hexanes).

Following experimental procedure D (Method B), N-alkylindoline 24 was prepared by dissolving cyclopropane $4 \mathbf{j}$ (100 mg, $0.446 \mathrm{mmol}), \mathrm{Yb}(\mathrm{OTf})_{3}(14 \mathrm{mg}, 0.022 \mathrm{mmol}$ ), and indoline 3a (106 mg, $0.892 \mathrm{mmol}$ ) in $5 \mathrm{~mL}$ of toluene. The reaction was hated at $100{ }^{\circ} \mathrm{C}$ for $2 \mathrm{~h}$. Compound 24 (122 mg, $0.356 \mathrm{mmol}, 80 \%$ ) was obtained as a yellow oil.

${ }^{1}$ HNMR $\left(400 \mathrm{MHz}, \mathrm{CDCl}_{3}\right): \delta=7.13(\mathrm{~d}, J=7.8 \mathrm{~Hz}, 2 \mathrm{H}), 7.04(\mathrm{dd}, J=7.8,2.4 \mathrm{~Hz}, 2 \mathrm{H}), 6.67$, (t, $J=7.0 \mathrm{~Hz}, 1 \mathrm{H}), 3.86(\mathrm{t}, J=6.6 \mathrm{~Hz}, 1 \mathrm{H}), 3.67(\mathrm{~s}, 3 \mathrm{H}), 3.62(\mathrm{~s}, 3 \mathrm{H}), 3.38-3.44(\mathrm{~m}, 2 \mathrm{H}), 2.86(\mathrm{t}, J$ $=8.9 \mathrm{~Hz}, 2 \mathrm{H}), 2.68(\mathrm{dd}, J=14.4,5.5 \mathrm{~Hz}, 1 \mathrm{H}), 2.46(\mathrm{dd}, J=14.1,6.6 \mathrm{~Hz}, 1 \mathrm{H}), 2.20(\mathrm{q}, J=7.4$ $\mathrm{Hz}, 2 \mathrm{H}), 1.49$ (s, 3H), 1.13 (t, $J=7.4 \mathrm{~Hz}, 3 \mathrm{H}) .{ }^{13} \mathrm{CNMR}\left(100 \mathrm{MHz}, \mathrm{CDCl}_{3}\right): \delta=169.9,169.6$, $149.2,131.5,126.4,124.0,117.9,111.6,86.8,80.2$, 54.0, 52.4, 49.6, 48.6, 38.0, 27.8, 23.3, 13.6, 12.2. FT-IR (thin film, $\mathrm{cm}^{-1}$ ): 3102, 3043, 2978, 2951, 2844, 2245, 1735, 1604, 1483, 1435, 1330, 1244, 1149, 748. HRMS calc'd for $\mathrm{C}_{20} \mathrm{H}_{25} \mathrm{NO}_{4}\left[\mathrm{M}^{+}\right]$: 343.1784; found: 343.1772.

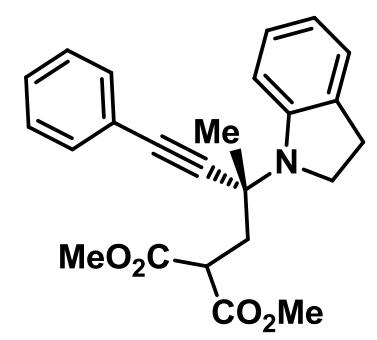

Dimethyl 2-(2-(indolin-1-yl)-2-methyl-4-phenylbut-3-ynyl)malonate (25). Following experimental procedure D (Method A), N-alkylindoline 25 was prepared by dissolving cyclopropane $4 \mathbf{k}$ (378 $\mathrm{mg}, 1.39 \mathrm{mmol}$ ), $\mathrm{Sc}(\mathrm{OTf})_{3}(68 \mathrm{mg}, 0.139 \mathrm{mmol})$, and indoline $3 \mathrm{a}(331 \mathrm{mg}, 2.78 \mathrm{mmol})$ in $10 \mathrm{~mL}$ of toluene. The reaction was hated at $100^{\circ} \mathrm{C}$ for $3 \mathrm{~h}$. Compound 25 (428 mg, $1.09 \mathrm{mmol}, 79 \%)$ was obtained as a yellow oil. $\mathrm{Rf}=0.55$ (30\% EtOAc in hexanes).

Following experimental procedure D (Method B), N-alkylindoline 25 was prepared by dissolving cyclopropane 4k (234 mg, $0.858 \mathrm{mmol}), \mathrm{Yb}(\mathrm{OTf})_{3}(27 \mathrm{mg}, 0.043 \mathrm{mmol})$, and indoline 
3a (204 mg, $1.72 \mathrm{mmol})$ in $10 \mathrm{~mL}$ of toluene. The reaction was hated at $100{ }^{\circ} \mathrm{C}$ for $3 \mathrm{~h}$. Compound 25 (122 mg, $0.356 \mathrm{mmol}, 79 \%$ ) was obtained as a yellow oil.

${ }^{1}$ HNMR $\left(400 \mathrm{MHz}, \mathrm{CDCl}_{3}\right): \delta=7.43(\mathrm{dd}, J=7.3,2.7 \mathrm{~Hz}, 2 \mathrm{H}), 7.32-7.28(\mathrm{~m}, 3 \mathrm{H}), 7.23(\mathrm{~d}, J=$ $8.2 \mathrm{~Hz}, 1 \mathrm{H}$ ), 7.08 (dd, $J=7.4,2.7 \mathrm{~Hz}, 1 \mathrm{H}$ ), 6.71 (t, $J=7.4 \mathrm{~Hz}, 1 \mathrm{H}$ ), 3.94 (dd, $J=7.0,5.5 \mathrm{~Hz}$, $1 \mathrm{H}), 3.62(\mathrm{~s}, 3 \mathrm{H}), 3.58(\mathrm{~s}, 3 \mathrm{H}), 3.50-3.42(\mathrm{~m}, 2 \mathrm{H}), 2.94-2.89(\mathrm{~m}, 2 \mathrm{H}), 2.79(\mathrm{dd}, J=14.5,5.5$ $\mathrm{Hz}, 1 \mathrm{H}), 2.61(\mathrm{dd}, J=14.5,5.5 \mathrm{~Hz}, 1 \mathrm{H}), 1.61(\mathrm{~s}, 3 \mathrm{H}) .{ }^{13} \mathrm{CNMR}\left(100 \mathrm{MHz}, \mathrm{CDCl}_{3}\right): \delta=169.9$, $169.7,149.3,131.6,128.2,126.7,124.3,122.6$, 118.4, 111.7, 90.7, 85.2, 54.6, 52.6, 49.7, 48.6, 38.3, 28.0, 23.0. FT-IR (thin film, $\mathrm{cm}^{-1}$ ): 3021, 2951, 2843, 1735, 1604, 1483, 1247, 1157. HRMS calc'd for $\mathrm{C}_{24} \mathrm{H}_{25} \mathrm{NO}_{4}\left[\mathrm{M}^{+}\right]$: 391.1784 ; found: 391.1770 .

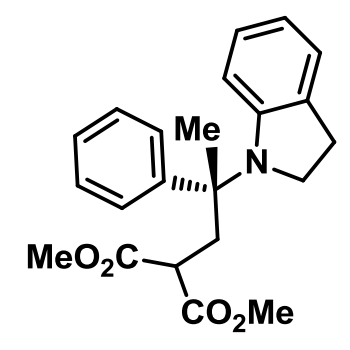

Dimethyl 2-(2-(indolin-1-yl)-2-phenylpropyl)malonate (26). Following experimental procedure $\mathrm{D}$ (Method A), $\mathrm{N}$-alkylindoline 26 was prepared by dissolving cyclopropane $4 \mathrm{l}$ (219 $\mathrm{mg}, 0.881 \mathrm{mmol}), \mathrm{Sc}(\mathrm{OTf})_{3}$ (43 $\mathrm{mg}, 0.088$ $\mathrm{mmol})$, and indoline $3 \mathrm{a}(210 \mathrm{mg}, 1.76 \mathrm{mmol})$ in $8 \mathrm{~mL}$ of toluene. The reaction was hated at $100{ }^{\circ} \mathrm{C}$ for $2.5 \mathrm{~h}$. Compound $26(246 \mathrm{mg}, 0.670$ mmol, 76\%) was obtained as a white solid. $\mathrm{Mp}=126-128{ }^{\circ} \mathrm{C} . \mathrm{Rf}=0.57(30 \%$ EtOAc in hexanes).

Following experimental procedure D (Method B), N-alkylindoline 26 was prepared by dissolving cyclopropane $4 \mathrm{l}$ (100 mg, $0.402 \mathrm{mmol}), \mathrm{Yb}(\mathrm{OTf})_{3}(13 \mathrm{mg}, 0.020 \mathrm{mmol})$, and indoline 3a (96 mg, $0.805 \mathrm{mmol}$ ) in $5 \mathrm{~mL}$ of toluene. The reaction was hated at $100{ }^{\circ} \mathrm{C}$ for $2.5 \mathrm{~h}$. Compound 26 (140 mg, $0.380 \mathrm{mmol}, 85 \%$ ) was obtained as a white solid.

${ }^{1} \mathrm{HNMR}\left(400 \mathrm{MHz}, \mathrm{CDCl}_{3}\right): \delta=7.47(\mathrm{~d}, J=8.6 \mathrm{~Hz}, 2 \mathrm{H}), 7.30(\mathrm{t}, J=7.8 \mathrm{~Hz}, 2 \mathrm{H}), 7.23(\mathrm{t}, J=7.0$ $\mathrm{Hz}, 1 \mathrm{H}$ ), $7.05(\mathrm{~d}, J=7.0 \mathrm{~Hz}, 1 \mathrm{H}), 6.67(\mathrm{t}, J=7.8 \mathrm{~Hz}, 1 \mathrm{H}), 6.56$ (t, $J=7.0,1 \mathrm{H}), 5.60(\mathrm{~d}, J=7.8$ $\mathrm{Hz}, 1 \mathrm{H}), 3.78-3.73(\mathrm{td}, J=8.6,2.4 \mathrm{~Hz}, 1 \mathrm{H}), 3.61(\mathrm{~s}, 3 \mathrm{H}), 3.56(\mathrm{t}, J=5.8 \mathrm{~Hz}, 1 \mathrm{H}), 3.52-3.44(\mathrm{~m}$, $1 \mathrm{H}), 3.39(\mathrm{~s}, 3 \mathrm{H}), 3.05-2.97(\mathrm{~m}, 2 \mathrm{H}), 2.64(\mathrm{~d}, J=5.8 \mathrm{~Hz}, 2 \mathrm{H}), 1.53(\mathrm{~s}, 3 \mathrm{H}) .{ }^{13} \mathrm{CNMR}(100 \mathrm{MHz}$, $\left.\mathrm{CDCl}_{3}\right): \delta=170.0169 .4,149.6,143.8,131.4,126.4,126.8,126.2,124.1,117.5,112.0,60.7$, 52.7, 52.3, 49.1, 47.7, 41.5, 28.3, 18.4. FT-IR (thin film, $\mathrm{cm}^{-1}$ ): 2951, 2842, 1752, 1734, 1604, 1484, 1435, 1244, 1202, 1158, 748, 702. HRMS calc'd for $\mathrm{C}_{22} \mathrm{H}_{25} \mathrm{NO}_{4}\left[\mathrm{M}^{+}\right]: 367.1784$; found: 367.1785 . 


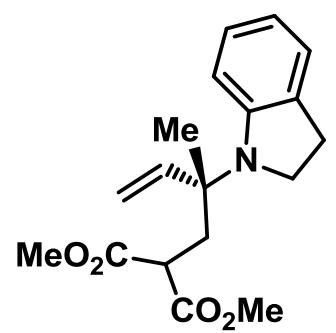

Dimethyl 2-(2-(indolin-1-yl)-2-methylbut-3-enyl)malonate (27). Following experimental procedure $\mathrm{D}$ (Method A), $\mathrm{N}$-alkylindoline 27 was prepared by dissolving cyclopropane $4 \mathrm{~m}$ (200 mg, $1.00 \mathrm{mmol}$ ), Sc(OTf) 3 (49 mg, 0.101 $\mathrm{mmol})$, and indoline $3 \mathrm{a}(240 \mathrm{mg}, 2.00 \mathrm{mmol})$ in $8 \mathrm{~mL}$ of toluene. The reaction was hated at $100{ }^{\circ} \mathrm{C}$ for $3 \mathrm{~h}$. Compound 27 (135 mg, $0.444 \mathrm{mmol}, 44 \%$ ) was obtained as a brown oil. $\mathrm{Rf}=0.55$ (30\% EtOAc in hexanes).

Following experimental procedure D (Method B), N-alkylindoline 27 was prepared by dissolving cyclopropane $4 \mathrm{~m}$ (436 mg, $2.20 \mathrm{mmol}), \mathrm{Yb}(\mathrm{OTf})_{3}(68 \mathrm{mg}, 0.110 \mathrm{mmol})$, and indoline 3a $(525 \mathrm{mg}, 4.40 \mathrm{mmol})$ in $10 \mathrm{~mL}$ toluene. The reaction was hated at $100{ }^{\circ} \mathrm{C}$ for $3 \mathrm{~h}$. Compound 27 (334 mg, $1.09 \mathrm{mmol}, 50 \%$ ) was obtained as a brown oil.

${ }^{1}$ HNMR $\left(400 \mathrm{MHz}, \mathrm{CDCl}_{3}\right): \delta=7.04(\mathrm{~d}, J=8.2 \mathrm{~Hz}, 1 \mathrm{H}), 6.93(\mathrm{t}, J=7.8 \mathrm{~Hz}, 1 \mathrm{H}), 6.73(\mathrm{~d}, J=$ $7.8 \mathrm{~Hz}, 1 \mathrm{H}$ ), $6.63(\mathrm{t}, J=8.2 \mathrm{~Hz}, 1 \mathrm{H}$ ), 5.96 (dd, $J=18.0,10.9 \mathrm{~Hz}, 1 \mathrm{H}), 5.19$ (dd, $J=11.7,9.7$ $\mathrm{Hz}, 2 \mathrm{H}), 3.71(\mathrm{~s}, 3 \mathrm{H}), 3.68(\mathrm{dd}, J=8.2,4.3 \mathrm{~Hz}, 1 \mathrm{H}), 3.59(\mathrm{~s}, 3 \mathrm{H}), 3.52-3.47(\mathrm{~m}, 1 \mathrm{H}), 3.35-3.28$ (m, 1H), 2.95-2.83 (m, 2H), 2.53 (dd, $J=14.8,8.2 \mathrm{~Hz}, 1 \mathrm{H}), 2.43(\mathrm{dd}, J=14.8,4.3 \mathrm{~Hz}, 1 \mathrm{H}$ ), 1.29 (s, 3H). ${ }^{13} \mathrm{CNMR}\left(100 \mathrm{MHz}, \mathrm{CDCl}_{3}\right): \delta=170.2,170.1,150.0,142.8,131.5,126.4,124.2$, 117.6, 114.7, 111.7, 59.4, 52.7, 52.3, 49.2, 47.7, 38.6, 28.0, 19.1. FT-IR (thin film, $\mathrm{cm}^{-1}$ ): 3272 , 2990, 2952, 2844, 1732, 1605, 1483, 1263, 1155. HRMS calc'd for $\mathrm{C}_{18} \mathrm{H}_{23} \mathrm{NO}_{4}\left[\mathrm{M}^{+}\right]$: 315.1471 ; found: 315.1474 .

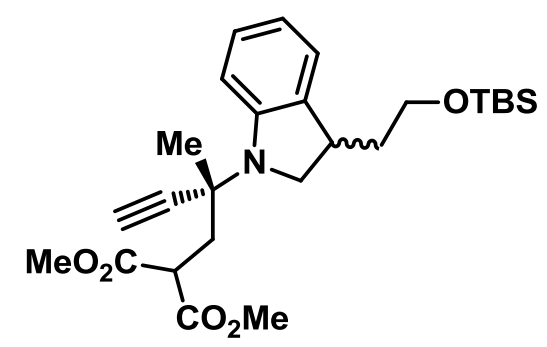

Dimethyl 2-(2-(3-(2-(tert-butyldimethylsilyloxy)ethyl)indolin1-yl)-2-methylbut-3-ynyl)malonate (28).

Following experimental procedure $\mathrm{D}$ (Method A), $\mathrm{N}$ alkylindoline $\mathbf{2 8}$ was prepared by dissolving cyclopropane $\mathbf{4 i}$ (100 mg, $0.510 \mathrm{mmol}), \mathrm{Sc}(\mathrm{OTf})_{3}(25 \mathrm{mg}, 0.051 \mathrm{mmol})$, and 3-(2(tert-butyldimethylsilyloxy)ethyl)indoline $3 \mathrm{c}^{5}(282 \mathrm{mg}, 1.01 \mathrm{mmol})$ in $5 \mathrm{~mL}$ of toluene. The reaction was hated at $100{ }^{\circ} \mathrm{C}$ for $1.5 \mathrm{~h}$. Compound $28(211 \mathrm{mg}, 0.448 \mathrm{mmol}, 80 \%)$ was obtained in a 1:1 mixture of diastereomers and as a brown oil. $\mathrm{Rf}=0.51$ (30\% EtOAc in hexanes). ${ }^{1} \mathrm{HNMR}$ (400 MHz, $\mathrm{CDCl}_{3}$ ) (mixture of diastereomers): $\delta=7.10-7.07$ (t, $J=6.3 \mathrm{~Hz}$, 2H), $7.05(\mathrm{~d}, J=5.1 \mathrm{~Hz}, 4 \mathrm{H}), 6.75-6.70(\mathrm{~m}, 2 \mathrm{H}), 3.86-3.82(\mathrm{~m}, 2 \mathrm{H}), 3.73(\mathrm{td}, J=6.3,3.1 \mathrm{~Hz}$, $4 \mathrm{H}), 3.68(\mathrm{~s}, 3 \mathrm{H}), 3.65(\mathrm{~s}, 3 \mathrm{H}), 3.62(\mathrm{~s}, 4 \mathrm{H}), 3.59(\mathrm{~s}, 4 \mathrm{H}), 3.23-3.19(\mathrm{~m}, 2 \mathrm{H}), 3.13(\mathrm{t}, J=8.21$ 
$\mathrm{Hz}, 1 \mathrm{H}), 3.08(\mathrm{t}, J=8.21 \mathrm{~Hz}, 1 \mathrm{H}), 2.80-2.67(\mathrm{~m}, 2 \mathrm{H}), 2.63-2.46(\mathrm{~m}, 2 \mathrm{H}), 2.44(\mathrm{~s}, 1 \mathrm{H}), 2.42(\mathrm{~s}$, $1 \mathrm{H}), 2.09-1.98(\mathrm{~m}, 2 \mathrm{H}), 1.76-1.68(\mathrm{~m}, 2 \mathrm{H}), 1.55(\mathrm{~s}, 3 \mathrm{H}), 1.53(\mathrm{~s}, 3 \mathrm{H}), 0.91(\mathrm{~s}, 18 \mathrm{H}), 0.08(\mathrm{~s}$, 12H). ${ }^{13} \mathrm{CNMR}\left(100 \mathrm{MHz}, \mathrm{CDCl}_{3}\right): \delta=169.9,169.8,169.6,169.5,148.7,148.5,135.3,126.8$, $126.7,123.5,123.2,118.5,118.4,111.7,111.6,85.1,84.9,73.3,61.5,61.4,56.4,56.3,54.2$, $53.9,52.6,52.4,48.6,48.4,38.4,37.5,36.9,36.8,36.7,36.6,25.9,23.6,23.5,18.3,18.2$, 5.3, -5.2. FT-IR (thin film, $\mathrm{cm}^{-1}$ ): 3273, 2952, 2930, 2856, 1736, 1482, 1436, 1256, 1094. HRMS calc'd for $\mathrm{C}_{26} \mathrm{H}_{39} \mathrm{NO}_{5} \mathrm{Si}\left[\mathrm{M}^{+}\right]$: 473.2597; found: 473.2599 .

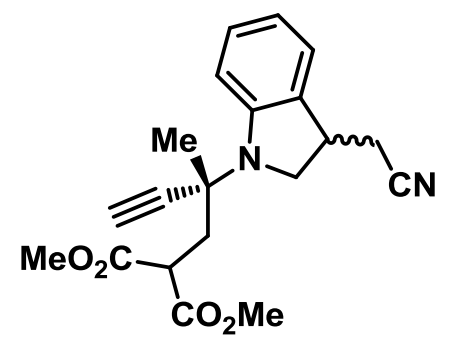

\section{Dimethyl 2-(2-(3-(cyanomethyl)indolin-1-yl)-2-methylbut-3- ynyl)malonate (29).}

Following experimental procedure D (Method A), N-alkylindoline 29 was prepared by dissolving cyclopropane $4 \mathbf{i}(390 \mathrm{mg}, 1.98 \mathrm{mmol}, 1$ equiv), $\mathrm{Sc}(\mathrm{OTf})_{3}(97 \mathrm{mg}, 0.198 \mathrm{mmol}, 10 \mathrm{~mol} \%$ ), and 2-(indolin-3$\mathrm{yl}$ )acetonitrile $3 \mathbf{d}^{6}$ (314 mg, $1.98 \mathrm{mmol}, 1$ equiv) in $10 \mathrm{~mL}$ of toluene. The reaction mixture was heated at $100{ }^{\circ} \mathrm{C}$ for 3 hours. Compound 29 (445 mg, 1.25mmol, 63\%) in a 1:1 mixture of diastereomers and as yellow oil. $\mathrm{Rf}=0.24\left(30 \%\right.$ EtOAc in hexanes). ${ }^{1} \mathrm{HNMR}\left(400 \mathrm{MHz}, \mathrm{CDCl}_{3}\right)$ (mixture of diastereomers) : $\delta=7.19(\mathrm{~d}, J=7.4 \mathrm{~Hz}, 2 \mathrm{H}), 7.15-7.11(\mathrm{~m}, 3 \mathrm{H}), 7.02(\mathrm{~d}, J=7.8$ $\mathrm{Hz}, 1 \mathrm{H}), 6.81-6.78(\mathrm{~m}, 2 \mathrm{H}), 3.83(\mathrm{t}, J=6.3 \mathrm{~Hz}, 1 \mathrm{H}), 3.79(\mathrm{t}, J=6.6 \mathrm{~Hz}, 1 \mathrm{H}), 3.68(\mathrm{~s}, 3 \mathrm{H}), 3.67$ (s, 3H), $3.61(\mathrm{~s}, 3 \mathrm{H}), 3.50(\mathrm{~s}, 3 \mathrm{H}), 3.47-3.42(\mathrm{~m}, 2 \mathrm{H}) .3 .39-3.31(\mathrm{~m}, 2 \mathrm{H}), 2.83(\mathrm{dd}, J=14.0,7.0$ $\mathrm{Hz}, 1 \mathrm{H}), 2.69-2.64(\mathrm{~m}, 2 \mathrm{H}), 2.64(\mathrm{~s}, 2 \mathrm{H}), 2.62(\mathrm{~s}, 1 \mathrm{H}), 2.49(\mathrm{~s}, 2 \mathrm{H}), 2.46(\mathrm{~s}, 1 \mathrm{H}), 1.59(\mathrm{~s}, 3 \mathrm{H} 0$, 1.57 (s, 3H). ${ }^{13} \mathrm{CNMR}\left(100 \mathrm{MHz}, \mathrm{CDCl}_{3}\right): \delta=169.6,169.6,169.5,169.3,148.3,147.9,131.5$, $131.2,128.3,128.2,123.9,123.8,119.2,119.1,118.5,112.3,112.1,84.6,84.2,73.7,55.8$, $54.9,54.6,53.9,52.7,52.6,52.5,52.4,48.8,48.3,38.4,38.0,36.6,36.5,23.8,23.2,21.8$, 21.9. FT-IR (thin film, $\mathrm{cm}^{-1}$ ): 3269, 2950, 2849, 2247, 1731, 1604, 1483, 1434, 1262, 1198 , 1154. HRMS calc'd for $\mathrm{C}_{20} \mathrm{H}_{22} \mathrm{~N}_{2} \mathrm{O}_{4}\left[\mathrm{M}^{+}\right]$: 354.1580 ; found: 354.1584 . 
Experimental Procedure E: $\mathrm{Mn}(\mathrm{OAc})_{3}$ Promoted Cyclization of $\mathrm{N}$-alkylindolines to pyrroloindoles

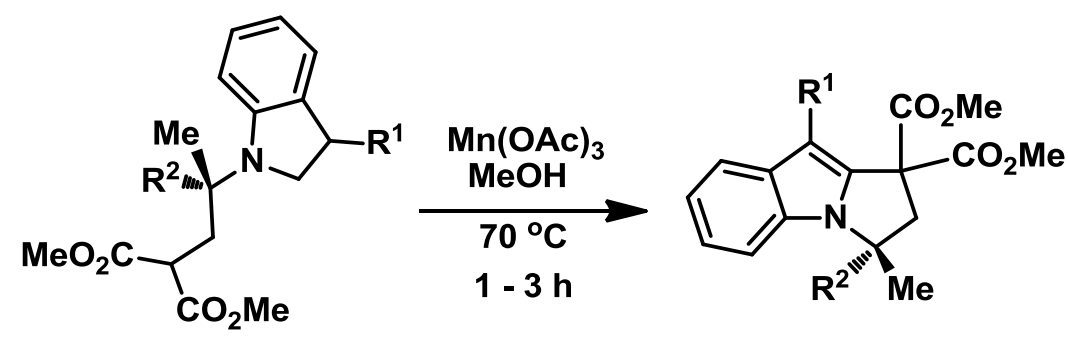

Compounds 30-36 were prepared according to the following procedure. To a solution of $\mathrm{N}$ alkylindoline 23-29 (1 equiv) in $8-15 \mathrm{~mL}$ of $\mathrm{MeOH}$ was added $\mathrm{Mn}(\mathrm{OAc})_{3}$ (5 equivs). The reaction mixture was heated at $70{ }^{\circ} \mathrm{C}$ for 1 - 3 hours (monitored by TLC). The crude reaction mixture was concentrated, pre-absorbed onto silica gel, and purified by column chromatography (EtOAc in Hexanes).

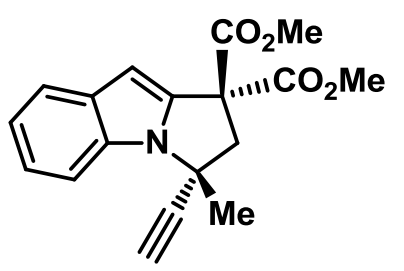

Dimethyl 3-ethynyl-3-methyl-2,3-dihydro-1H-pyrrolo[1,2-a]indole1,1-dicarboxylate (30).

Following experimental procedure $\mathrm{E}$, pyrroloindole $\mathbf{3 0}$ was prepared by dissolving $\mathrm{N}$-alkylindoline 23 (618 $\mathrm{mg}, 1.96 \mathrm{mmol})$ and $\mathrm{Mn}(\mathrm{OAc})_{3}(2620$ $\mathrm{mg}, 9.79 \mathrm{mmol}$ ) in $15 \mathrm{~mL}$ of $\mathrm{MeOH}$. The reaction was hated at $70{ }^{\circ} \mathrm{C}$ for $1 \mathrm{~h}$. Compound 30 (397 mg, $1.28 \mathrm{mmol}, 65 \%)$ was obtained as a brown solid. $\mathrm{Mp}=82-84{ }^{\circ} \mathrm{C} . \mathrm{Rf}=0.53(30 \%$ EtOAc in hexanes). ${ }^{1} \mathrm{HNMR}\left(400 \mathrm{MHz}, \mathrm{CDCl}_{3}\right): \delta=7.62(\mathrm{~d}, J=7.8 \mathrm{~Hz}, 1 \mathrm{H}), 7.57(\mathrm{~d}, J=7.8$ $\mathrm{Hz}, 1 \mathrm{H}), 7.21(\mathrm{td}, J=7.8,1.2 \mathrm{~Hz}, 1 \mathrm{H}), 7.13(\mathrm{td}, J=7.8,1.2 \mathrm{~Hz}, 1 \mathrm{H}), 6.51(\mathrm{~s}, 3 \mathrm{H}), 3.82(\mathrm{~s}, 6 \mathrm{H})$, $3.63(\mathrm{~d}, J=13.6 \mathrm{~Hz}, 1 \mathrm{H}), 3.37(\mathrm{~d}, J=13.6 \mathrm{~Hz}, 1 \mathrm{H}), 2.54(\mathrm{~s}, 1 \mathrm{H}), 1.90(\mathrm{~s}, 3 \mathrm{H}) \cdot{ }^{13} \mathrm{CNMR}(100$ $\left.\mathrm{MHz}, \mathrm{CDCl}_{3}\right): \delta=169.3,168.9,137.6,133.0,131.2,121.8,121.7,120.1,110.3,97.3,83.9$, 72.4, 58.2, 55.1, 53.5, 51.7, 28.1. FT-IR (thin film, $\mathrm{cm}^{-1}$ ): 3275, 2953, 2112, 1741, 1449, 1256, 1159. HRMS calc'd for $\mathrm{C}_{18} \mathrm{H}_{17} \mathrm{NO}_{4}\left[\mathrm{M}^{+}\right]: 311.1158$; found: 311.1153 .

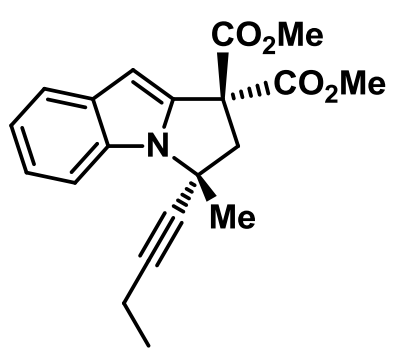

Dimethyl 3-(but-1-ynyl)-3-methyl-2,3-dihydro-1H-pyrrolo[1,2a]indole-1,1-dicarboxylate (31).

Following experimental procedure $\mathrm{E}$, pyrroloindole 31 was prepared by dissolving $\mathrm{N}$-alkylindoline 24 (276 $\mathrm{mg}, 0.804 \mathrm{mmol})$ and $\mathrm{Mn}(\mathrm{OAc})_{3}$ (1070 mg, $4.02 \mathrm{mmol}$ ) in $8 \mathrm{~mL}$ of $\mathrm{MeOH}$. The reaction was hated at 70 ${ }^{\circ} \mathrm{C}$ for $1.5 \mathrm{~h}$. Compound 31 (177 mg, $0.523 \mathrm{mmol}, 65 \%$ ) was obtained as a yellow oil. $\mathrm{Rf}=0.51$ 
(30\% EtOAc in hexanes). ${ }^{1} \mathrm{HNMR}\left(400 \mathrm{MHz}, \mathrm{CDCl}_{3}\right): \delta=7.59(\mathrm{t}, \mathrm{J}=8.2 \mathrm{~Hz}, 2 \mathrm{H}), 7.18(\mathrm{td}, \mathrm{J}=$ 7.0, 1.2 Hz, 1H), $7.10(\mathrm{td}, J=7.0,1.2 \mathrm{~Hz}, 1 \mathrm{H}), 6.47(\mathrm{~s}, 1 \mathrm{H}), 3.81$ (s, 3H), $3.80(\mathrm{~s}, 3 \mathrm{H}), 3.55$ (d, $J=13.7 \mathrm{~Hz}, 1 \mathrm{H}$ ), $3.32(\mathrm{~d}, J=13.7 \mathrm{~Hz}, 1 \mathrm{H}), 2.20(\mathrm{q}, J=7.4 \mathrm{~Hz}, 2 \mathrm{H}), 1.81(\mathrm{~s}, 3 \mathrm{H}), 1.12(\mathrm{t}, J=$ $7.4 \mathrm{~Hz}, 3 \mathrm{H}) .{ }^{13} \mathrm{CNMR}\left(100 \mathrm{MHz}, \mathrm{CDCl}_{3}\right): \delta=169.5,169.0,137.5,132.9,131.2,121.5,119.8$, 110.5, 96.8, 86.2, 79.9, 58.3, 55.5, 53.5, 52.0, 28.6, 13.7, 12.3. FT-IR (thin film, $\mathrm{cm}^{-1}$ ): 2978, 2952, 2845, 2242, 1742, 1449, 1254, 1161. HRMS calc'd for $\mathrm{C}_{20} \mathrm{H}_{21} \mathrm{NO}_{4}\left[\mathrm{M}^{+}\right]$: 339.1471 ; found: 339.1458.

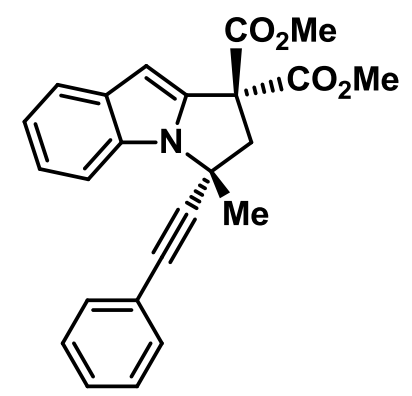

\section{Dimethyl 3-methyl-3-(phenylethynyl)-2,3-dihydro-1H-pyrrolo[1,2- a]indole-1,1-dicarboxylate (32).}

Following experimental procedure $\mathrm{E}$, pyrroloindole 32 was prepared by dissolving $\mathrm{N}$-alkylindoline 25 (369 $\mathrm{mg}, 0.943 \mathrm{mmol}$ ) and $\mathrm{Mn}(\mathrm{OAc})_{3}$ (1260 mg, $4.72 \mathrm{mmol})$ in $10 \mathrm{~mL}$ of $\mathrm{MeOH}$. The reaction was hated at 70 ${ }^{\circ} \mathrm{C}$ for $1.5 \mathrm{~h}$. Compound 32 (225 mg, $0.581 \mathrm{mmol}$ ) was obtained as a yellow solid $(61 \%)$. $\mathrm{Mp}=$ $108-110{ }^{\circ} \mathrm{C} \mathrm{Rf}=0.48\left(30 \%\right.$ EtOAc in hexanes). ${ }^{1} \mathrm{HNMR}\left(400 \mathrm{MHz}, \mathrm{CDCl}_{3}\right): \delta=7.65-7.62(\mathrm{~m}$, 2H), 7.42-7.39 (m, 2H), 7.32-7.27 (m, 3H), $7.21(\mathrm{td}, J=7.0,1.2 \mathrm{~Hz}, 1 \mathrm{H}), 7.13(\mathrm{td}, J=7.0,1.2$ $\mathrm{Hz}, 1 \mathrm{H}), 6.52(\mathrm{~s}, 1 \mathrm{H}), 3.83(\mathrm{~s}, 3 \mathrm{H}), 3.82(\mathrm{~s}, 3 \mathrm{H}), 3.70(\mathrm{~d}, J=13.6 \mathrm{~Hz}, 1 \mathrm{H}), 3.44(\mathrm{~d}, J=13.6 \mathrm{~Hz}$, $1 \mathrm{H}), 1.96(\mathrm{~s}, 3 \mathrm{H}) .{ }^{13} \mathrm{CNMR}\left(100 \mathrm{MHz}, \mathrm{CDCl}_{3}\right): \delta=169.4,168.9,137.5,132.9,131.7,131.3$, 128.5, 128.3, 122.2, 121.7, 121.5, 120.0, 110.4, 97.1, 89.3, 84.2, 58.3, 55.8, 53.6, 53.5, 51.9, 28.3. FT-IR (thin film, $\mathrm{cm}^{-1}$ ): 2996, 2952, 1742, 1449, 1263, 1168. HRMS calc'd for $\mathrm{C}_{24} \mathrm{H}_{21} \mathrm{NO}_{4}$ $\left[\mathrm{M}^{+}\right]$: 387.1471 ; found: 387.1475 .

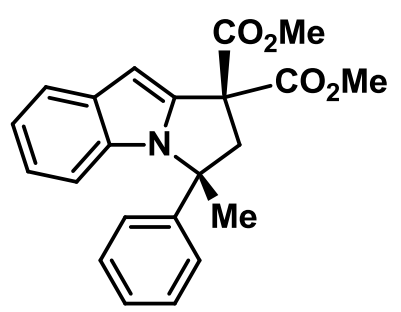

Dimethyl 3-methyl-3-phenyl-2,3-dihydro-1H-pyrrolo[1,2-a]indole1,1-dicarboxylate (33).

Following experimental procedure $\mathrm{E}$, pyrroloindole $\mathbf{3 3}$ was prepared by dissolving $N$-alkylindoline 26 (344 $\mathrm{mg}, 0.936 \mathrm{mmol}$ ) and $\mathrm{Mn}(\mathrm{OAc})_{3}$ (1250 mg, $4.68 \mathrm{mmol}$ ) in $10 \mathrm{~mL}$ of $\mathrm{MeOH}$. The reaction was hated at $70{ }^{\circ} \mathrm{C}$ for $2 \mathrm{~h}$. Compound $33(284 \mathrm{mg}, 0.781 \mathrm{mmol}, 83 \%)$ was obtained as colourless oil. $\mathrm{Rf}=0.53$ (30\% EtOAc in hexanes). ${ }^{1} \mathrm{HNMR}\left(400 \mathrm{MHz}, \mathrm{CDCl}_{3}\right): \delta=7.67(\mathrm{~d}, J=7.8 \mathrm{~Hz}, 1 \mathrm{H}), 7.32-7.27(\mathrm{~m}, 3 \mathrm{H}), 7.12(\mathrm{dt}$, $J=8.2,1.9 \mathrm{~Hz}, 3 \mathrm{H}$ ), $7.02(\mathrm{dd}, J=8.2,1.1 \mathrm{~Hz}, 1 \mathrm{H}), 6.94(\mathrm{~d}, J=8.2 \mathrm{~Hz}, 1 \mathrm{H}) 6.60(\mathrm{~s}, 1 \mathrm{H}), 3.86$ (s, 3H), $3.62(\mathrm{~s}, 3 \mathrm{H}), 3.51(\mathrm{~d}, J=13.6 \mathrm{~Hz}, 1 \mathrm{H}), 3.42(\mathrm{~d}, J=13.6 \mathrm{~Hz}, 1 \mathrm{H}) 2.02(\mathrm{~s}, 3 \mathrm{H}) .{ }^{13} \mathrm{CNMR}$ $\left(100 \mathrm{MHz}, \mathrm{CDCl}_{3}\right): \delta=169.4,169.3,144.4,139.1,133.0,131.6,128.6,127.4,125.3,121.5$, 
119.8, 110.9, 96.8, 65.2, 58.5, 54.5, 53.5, 53.2, 26.2. FT-IR (thin film, $\mathrm{cm}^{-1}$ ): 3056, 2982, 2952 , 2842, 1739, 1610, 1448, 1257, 1165, 1094. HRMS calc'd for $\mathrm{C}_{22} \mathrm{H}_{21} \mathrm{NO}_{4}\left[\mathrm{M}^{+}\right]: 363.1471$; found: 363.1466 .

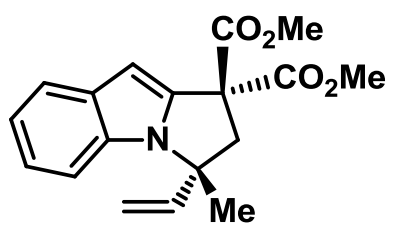

\section{Dimethyl 3-methyl-3-vinyl-2,3-dihydro-1H-pyrrolo[1,2-a]indole-1,1- dicarboxylate (34).}

Following experimental procedure $\mathrm{E}$, pyrroloindole $\mathbf{3 4}$ was prepared by dissolving $N$-alkylindoline 27 (398 mg, $1.31 \mathrm{mmol})$ and $\mathrm{Mn}(\mathrm{OAc})_{3}(1750 \mathrm{mg}, 6.53 \mathrm{mmol})$ in 10 $\mathrm{mL}$ of $\mathrm{MeOH}$. The reaction was hated at $70{ }^{\circ} \mathrm{C}$ for $1 \mathrm{~h}$. Compound $34(176 \mathrm{mg}, 0.563 \mathrm{mmol}$, $43 \%)$ was obtained as a colourless oil. $\mathrm{Rf}=0.57$ (30\% EtOAc in hexanes). ${ }^{1} \mathrm{HNMR}(400 \mathrm{MHz}$, $\left.\mathrm{CDCl}_{3}\right): \delta=7.61(\mathrm{~d}, J=8.2 \mathrm{~Hz}, 1 \mathrm{H}), 7.30(\mathrm{~d}, J=8.2 \mathrm{~Hz}, 1 \mathrm{H}), 7.14-7.07(\mathrm{~m}, 2 \mathrm{H}), 6.50(\mathrm{~s}, 1 \mathrm{H})$, 6.08 (dd, $J=17.5,10.5 \mathrm{~Hz}, 1 \mathrm{H}$ ), $5.18(\mathrm{~d}, J=10.5 \mathrm{~Hz}, 1 \mathrm{H}$ ), $5.02(\mathrm{~d}, J=17.2 \mathrm{~Hz}, 1 \mathrm{H}) 3.82$ (s, $3 \mathrm{H}), 3.77(\mathrm{~s}, 3 \mathrm{H}), 3.24(\mathrm{~d}, J=13.6 \mathrm{~Hz}, 1 \mathrm{H}), 3.18(\mathrm{~d}, J=13.6 \mathrm{~Hz}, 1 \mathrm{H}) 1.76(\mathrm{~s}, 3 \mathrm{H}) .{ }^{13} \mathrm{CNMR}$ $\left(100 \mathrm{MHz}, \mathrm{CDCl}_{3}\right): \delta=169.6,169.5,141.2,138.5,132.6,131.6,121.4,121.3,119.6,114.4$, 110.5, 96.6, 63.3, 58.3, 53.5, 53.4, 50.9, 24.2. FT-IR (thin film, $\mathrm{cm}^{-1}$ ): 2986, 2953, 2881, 1741 , 1449, 1256, 1144, 1095. HRMS calc'd for $\mathrm{C}_{18} \mathrm{H}_{19} \mathrm{NO}_{4}\left[\mathrm{M}^{+}\right]$: 313.1314 ; found: 313.1315.

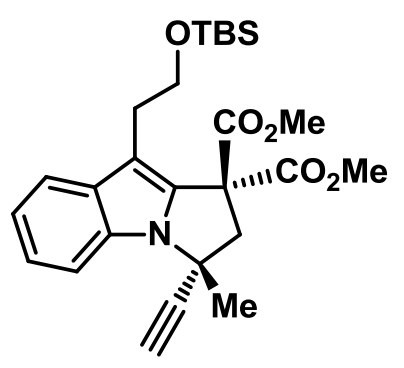

Dimethyl 9-(2-(tert-butyldimethylsilyloxy)ethyl)-3-ethynyl-3-methyl2,3-dihydro-1H-pyrrolo[1,2-a]indole-1,1-dicarboxylate (35).

Following experimental procedure $\mathrm{E}$, pyrroloindole $\mathbf{3 5}$ was prepared by dissolving $\mathrm{N}$-alkylindoline 28 (198 $\mathrm{mg}, 0.377 \mathrm{mmol}$ ) and $\mathrm{Mn}(\mathrm{OAc})_{3}(506$ $\mathrm{mg}, 1.88 \mathrm{mmol})$ in $8 \mathrm{~mL}$ of $\mathrm{MeOH}$. The reaction was hated at $70{ }^{\circ} \mathrm{C}$ for $3 \mathrm{~h}$. Compound 35 (142 mg, $0.302 \mathrm{mmol}, 80 \%$ ) was obtained as a colourless oil. $\mathrm{Rf}=0.57$ (30\% EtOAc in hexanes). ${ }^{1} \mathrm{HNMR}\left(400 \mathrm{MHz}, \mathrm{CDCl}_{3}\right): \delta=7.66(\mathrm{~d}, J=7.8 \mathrm{~Hz}, 1 \mathrm{H}), 7.54(\mathrm{~d}, J=$ $7.8 \mathrm{~Hz}, 1 \mathrm{H}$ ), 7.22 (t, $J=7.4 \mathrm{~Hz}, 1 \mathrm{H}$ ), 7.14 (t, $J=7.4 \mathrm{~Hz}, 1 \mathrm{H}$ ), 3.85 (dd, $J=7.0,3.9 \mathrm{~Hz}, 2 \mathrm{H}$ ), $3.82(\mathrm{~s}, 6 \mathrm{H}), 3.59$ (d, $J=13.2 \mathrm{~Hz}, 1 \mathrm{H}), 3.40$ (d, $J=13.2 \mathrm{~Hz}, 1 \mathrm{H}) 3.06$ (dd, $J=7.0,3.9 \mathrm{~Hz}, 2 \mathrm{H}$ ), $2.52(\mathrm{~s}, 1 \mathrm{H}), 1.87(\mathrm{~s}, 3 \mathrm{H}), 0.93(\mathrm{~s}, 9 \mathrm{H}), 0.08(\mathrm{~s}, 6 \mathrm{H}) .{ }^{13} \mathrm{CNMR}\left(100 \mathrm{MHz}, \mathrm{CDCl}_{3}\right): \delta=169.4$, 169.0, 134.0, 133.2, 130.1, 121.8, 120.2, 119.6, 110.2, 107.3, 83.9, 72.4, 63.2, 58.3, 54.6, 53.4, 53.3, 52.5, 28.2, 27.9. 26.0. 18.4. -5.2. FT-IR (thin film, $\mathrm{cm}^{-1}$ ): 3279, 2954, 2930, 2856, 1742, 1453, 1434, 1253, 1152, 1090. HRMS calc'd for $\mathrm{C}_{26} \mathrm{H}_{35} \mathrm{NO}_{5} \mathrm{Si}\left[\mathrm{M}^{+}\right]$: 469.2284; found: 469.2283. 


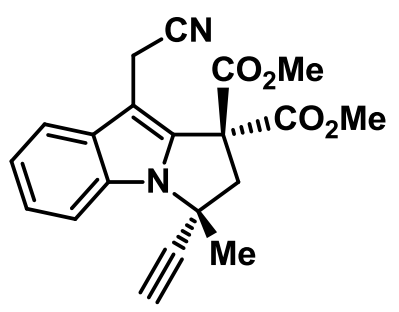

\section{Dimethyl 9-(cyanomethyl)-3-ethynyl-3-methyl-2,3-dihydro-1H- pyrrolo[1,2-a]indole-1,1-dicarboxylate (36).}

Following experimental procedure $\mathrm{E}$, pyrroloindole $\mathbf{3 6}$ was prepared by dissolving $\mathrm{N}$-alkylindoline 29 (444 $\mathrm{mg}, 1.25 \mathrm{mmol}$ ) and $\mathrm{Mn}(\mathrm{OAc})_{3}(1675$ $\mathrm{mg}, 6.25 \mathrm{mmol}$ ) in $10 \mathrm{~mL}$ of $\mathrm{MeOH}$. The reaction was hated at $70{ }^{\circ} \mathrm{C}$ for $3 \mathrm{~h}$. Compound 36 (150 mg, $0.428 \mathrm{mmol}, 35 \%)$ was obtained as a yellow solid. $\mathrm{Mp}=88-90{ }^{\circ} \mathrm{C} . \mathrm{Rf}=0.31(30 \%$ EtOAc in hexanes). ${ }^{1} \mathrm{HNMR}\left(400 \mathrm{MHz}, \mathrm{CDCl}_{3}\right): \delta=7.70(\mathrm{~d}, J=7.8 \mathrm{~Hz}, 1 \mathrm{H}), 7.60(\mathrm{~d}, J=8.2$ $\mathrm{Hz}, 1 \mathrm{H}), 7.30(\mathrm{t}, J=8.2 \mathrm{~Hz}, 1 \mathrm{H}), 7.24(\mathrm{t}, J=7.8 \mathrm{~Hz}, 1 \mathrm{H}), 3.97(\mathrm{~d}, J=8.2 \mathrm{~Hz}, 2 \mathrm{H}), 3.88(\mathrm{~d}, 6 \mathrm{H})$, $3.59(\mathrm{~d}, J=13.6 \mathrm{~Hz}, 1 \mathrm{H}), 3.47(\mathrm{~d}, J=13.6 \mathrm{~Hz}, 1 \mathrm{H}), 2.59(\mathrm{~s}, 1 \mathrm{H}), 1.90(\mathrm{~s}, 3 \mathrm{H}) .{ }^{13} \mathrm{CNMR}(100$ $\left.\mathrm{MHz}, \mathrm{CDCl}_{3}\right): \delta=168.7,168.5,134.8,131.9,131.1,123.1,120.9,119.3,118.4,110.9,99.3$, 83.8, 73.2, 58.7, 55.5, 54.2, 54.0, 51.6, 28.4, 13.3.FT-IR (thin film, $\mathrm{cm}^{-1}$ ): 3271, 2988, 2951, 1731, 1453, 1348, 1254, 1149, 1096, 745. HRMS calc'd for $\mathrm{C}_{20} \mathrm{H}_{18} \mathrm{~N}_{2} \mathrm{O}_{4}\left[\mathrm{M}^{+}\right]: 350.1267$; found: 350.1278 .

Hydrostannylation of Compound 35
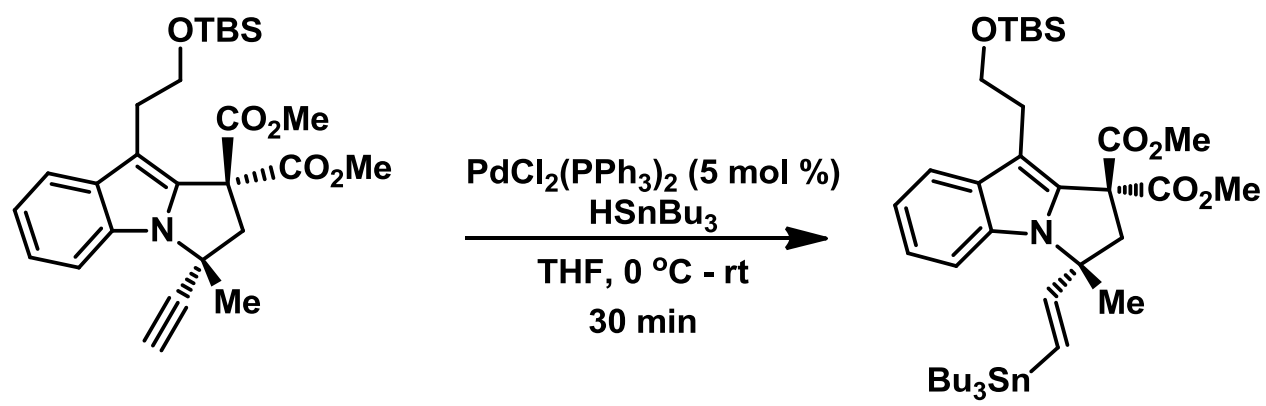

(E)-dimethyl 9-(2-(tert-butyldimethylsilyloxy)ethyl)-3-methyl-3-(2-(tributylstannyl)vinyl)2,3-dihydro-1H-pyrrolo[1,2-a]indole-1,1-dicarboxylate (37).

To a solution of compound 37 (50 mg, $0.106 \mathrm{mmol}, 1$ equiv) in $5 \mathrm{~mL}$ of THF, $\mathrm{PdCl}_{2}\left(\mathrm{PPh}_{3}\right)_{2}$ (3.72 $\mathrm{mg}, 0.005 \mathrm{mmol}, 5 \mathrm{~mol} \%$ ) was added and the mixture was cooled to $0{ }^{\circ} \mathrm{C}$. $\mathrm{Bu}_{3} \mathrm{SH}(34$ $\mathrm{mg}, 0.117 \mathrm{mmol}, 1.1$ equiv) was then added dropwise over a $5 \mathrm{~min}$ period. The mixture was stirred at $0{ }^{\circ} \mathrm{C}$ for 10 minutes and then at room temperature for 30 minutes. The crude reaction mixture was concentrated, pre-absorbed onto silica gel, and purified by column chromatography (EtOAc in hexanes). Compound 37 (68 mg, $0.089 \mathrm{mmol}, 85 \%$ ) was obtained 
as a colourless oil. $\mathrm{Rf}=0.77\left(30 \%\right.$ EtOAc in hexanes). ${ }^{1} \mathrm{HNMR}\left(400 \mathrm{MHz}, \mathrm{CDCl}_{3}\right): \delta=7.64(\mathrm{t}, J$ $=4.7 \mathrm{~Hz}, 1 \mathrm{H}), 7.22(\mathrm{t}, J=4.3 \mathrm{~Hz}, 1 \mathrm{H}), 7.08(\mathrm{dd}, J=4.7,4.3 \mathrm{~Hz}, 2 \mathrm{H}), 6.18(\mathrm{~d}, J=19.5 \mathrm{~Hz}, 1 \mathrm{H})$, $6.07(\mathrm{~d}, J=19.5 \mathrm{~Hz}, 1 \mathrm{H}), 3.87-3.82(\mathrm{~m}, 2 \mathrm{H}), 3.81(\mathrm{~s}, 3 \mathrm{H}), 3.76(\mathrm{~s}, 3 \mathrm{~h}), 3.19(\mathrm{~s}, 1 \mathrm{H}), 3.06(\mathrm{dd}$, $J=6.6 \mathrm{~Hz}, 2 \mathrm{H}), 1.68(\mathrm{~s}, 3 \mathrm{H}), 1.51-1.38(\mathrm{~m}, 6 \mathrm{H}), 1.30(\mathrm{~m}, 6 \mathrm{H}), 0.93(\mathrm{~s}, 9 \mathrm{H}), \quad 0.90-0.85(\mathrm{~m}$, $15 \mathrm{H}), 0.07(\mathrm{~s}, 6 \mathrm{H}) .{ }^{13} \mathrm{CNMR}\left(100 \mathrm{MHz}, \mathrm{CDCl}_{3}\right): \delta=169.9,169.6,150.1,135.2,132.9,131.4$, $131.3,128.0,121.3,119.9,118.9,110.5,106.3,102.8,101.4,64.3,63.3,58.5,58.3,53.2$, $53.1,52.1,29.1,29.0,28.8,28.3,37.3,27.2,26.0,23.8$, , $18.5,13.7,13.6,10.3,9.5,-5.2$. FTIR (thin film, $\mathrm{cm}^{-1}$ ): 2954, 2927, 2854, 1743, 1454, 1251, 1150, 1090. HRMS calc'd for $\mathrm{C}_{38} \mathrm{H}_{63} \mathrm{NO}_{5} \mathrm{SiSn}\left[\mathrm{M}^{+}\right]:$761.3497; found: 761.3587.

\section{Stille Coupling of Vinnylstannane 37}
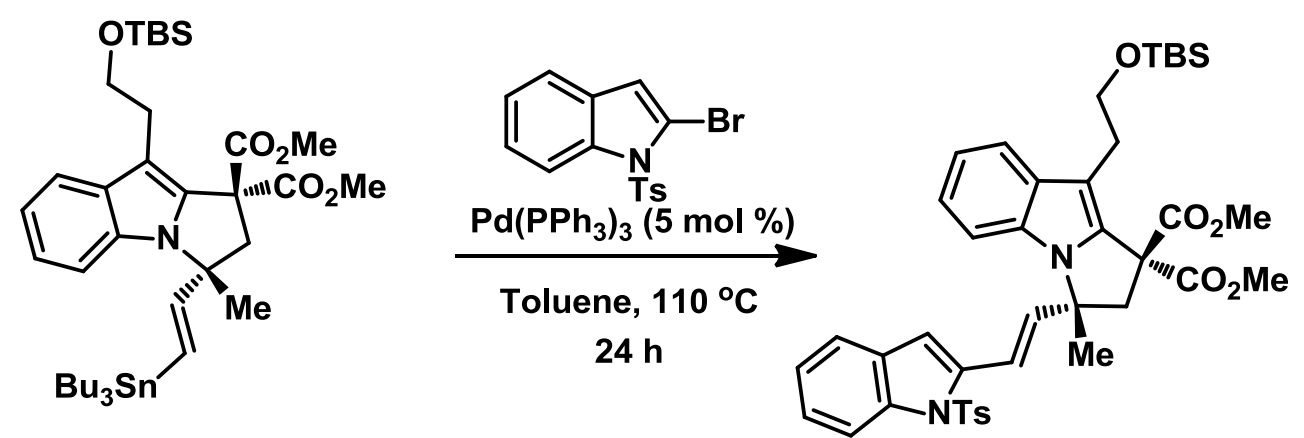

(E)-dimethyl 9-(2-(tert-butyldimethylsilyloxy)ethyl)-3-methyl-3-(2-(1-tosyl-1H-indol-2yl)vinyl)-2,3-dihydro-1H-pyrrolo[1,2-a]indole-1,1-dicarboxylate (39).

To a solution of compound 38 (39 mg, $0.051 \mathrm{mmol}, 1.1$ equivs) in $5 \mathrm{~mL}$ of toluene were added $\mathrm{Pd}\left(\mathrm{PPh}_{3}\right)_{4}\left(1.76 \mathrm{mg}, 0.001 \mathrm{mmol}, 3 \mathrm{~mol} \%\right.$ ) and 2-bromo-1-tosyl-1H-indole $38^{7}$ (16 mg, 0.046 mmol, 1 equiv). The reaction mixture was heated at $90{ }^{\circ} \mathrm{C}$ for 24 hours. The crude reaction mixture was concentrated, pre-absorbed onto silica gel, and purified by column chromatography (EtOAc in hexanes). Compound 39 (20 mg, $0.026 \mathrm{mmol}, 58 \%)$ was obtained as a colourless oil. $\mathrm{Rf}=0.31$ (30\% EtOAc in hexanes). ${ }^{1} \mathrm{HNMR}\left(400 \mathrm{MHz}, \mathrm{CDCl}_{3}\right): \delta=8.17(\mathrm{~d}$, $J=8.6 \mathrm{~Hz}, 1 \mathrm{H}), 7.71-7.69(\mathrm{~m} \mathrm{1H}), 7.45(\mathrm{~d}, 8.6 \mathrm{~Hz}, 2 \mathrm{H}), 7.40(\mathrm{~d}, J=7.8 \mathrm{~Hz}, 1 \mathrm{H}), 7.37-7.35(\mathrm{~m}$, $1 \mathrm{H}), 7.30(\mathrm{~d}, J=8.2 \mathrm{~Hz}, 1 \mathrm{H}) 7.21(\mathrm{~d}, J=7.8 \mathrm{~Hz}, 1 \mathrm{H}), 7.14-7.12(\mathrm{~m}, 2 \mathrm{H}), 7.07(\mathrm{~d}, J=8.2 \mathrm{~Hz}$, 2H), $7.03(\mathrm{~d}, J=16.0 \mathrm{~Hz}, 1 \mathrm{H}), 6.66(\mathrm{~s}, 1 \mathrm{H}), 6.36(\mathrm{~d}, J=16.0 \mathrm{~Hz}, 1 \mathrm{H}), 3.90(\mathrm{t}, J=8.2 \mathrm{~Hz}, 2 \mathrm{H})$ 3.85 (s, 3H), 3.74 (s, 3H), 3.40 (d, J=13.4 Hz, 1H), 3.27 (d, $J=13.4 \mathrm{~Hz}, 1 \mathrm{H}$ ), 3.10 (t, $J=8.2$ $\mathrm{Hz}, 2 \mathrm{H}), 2.31(\mathrm{~s}, 3 \mathrm{H}), 1.94(\mathrm{~s}, 3 \mathrm{H}), 0.94(\mathrm{~s}, 9 \mathrm{H}), 0.10(\mathrm{~s}, 6 \mathrm{H}) .{ }^{13} \mathrm{CNMR}\left(100 \mathrm{MHz}, \mathrm{CDCl}_{3}\right): \delta=$ $169.8,169.6,144.6,137.9,137.3,136.7,135.5,134.9,133.1,131.3,129.6,126.5,124.7$, 
$123.8,121.7,120.7,120.4,120.1,119.3,114.9,110.3109 .1,106.8,63.2,62.6,58.4,53.4$, 53.3, 52.2, 28.4, 26.1, 24.3, 21.5, 18.5, -5.2. FT-IR (thin film, $\mathrm{cm}^{-1}$ ): 2954, 2929, 2856, 1740, 1597, 1451, 1373, 1255, 1174, 1090. HRMS calc'd for $\mathrm{C}_{41} \mathrm{H}_{48} \mathrm{~N}_{2} \mathrm{O}_{7} \mathrm{SSi}\left[\mathrm{M}^{+}\right]$: 740.2951 ; found: 740.2961 .

\section{References}

1. Gross,E.; Liu, H-C.; Toste, F.D.; Somorjai, G.A. Nat. Chem. 2012, 4, 947-952.

2. Hatakeyama, T., Y., Y.; Gabriel, T.; Nakamura, M. Org. Lett. 2008, 10, 5341-5344.

3. Magolan, J.; Kerr, M.A. Org. Lett. 2006, 8, 4561-4564.

4. Johnson, K.F.; Van Zeeland, R.; Stanley, L.M. Org. Lett. 2013, 15, 2798-2801.

5. Hirose, T.; Sanazuka, T.; Yamamoto, D.; Kojima, N.; Shirahata, T.; Harigaya, Y.; Kuwajima, I.; Omura, S. Tetrahedron 2005, 61, 6015-6039.

6. Pedras, M.S.C.; Sarwar, M.G.; Suchy, M.; Adio, A.M. Phytochemistry, 2006, 67, 1503-1509.

7. Jiang, B.; Tao, K.; Shen, W.; Zhang, J. Tetrahedron Lett. 2010, 51, 6342-6344. 


\section{${ }^{1} \mathrm{HNMR}$ and ${ }^{13} \mathrm{CNMR}$ Spectra}




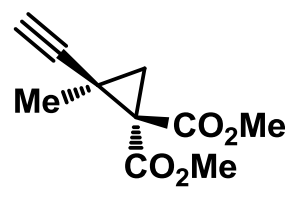

$4 i$

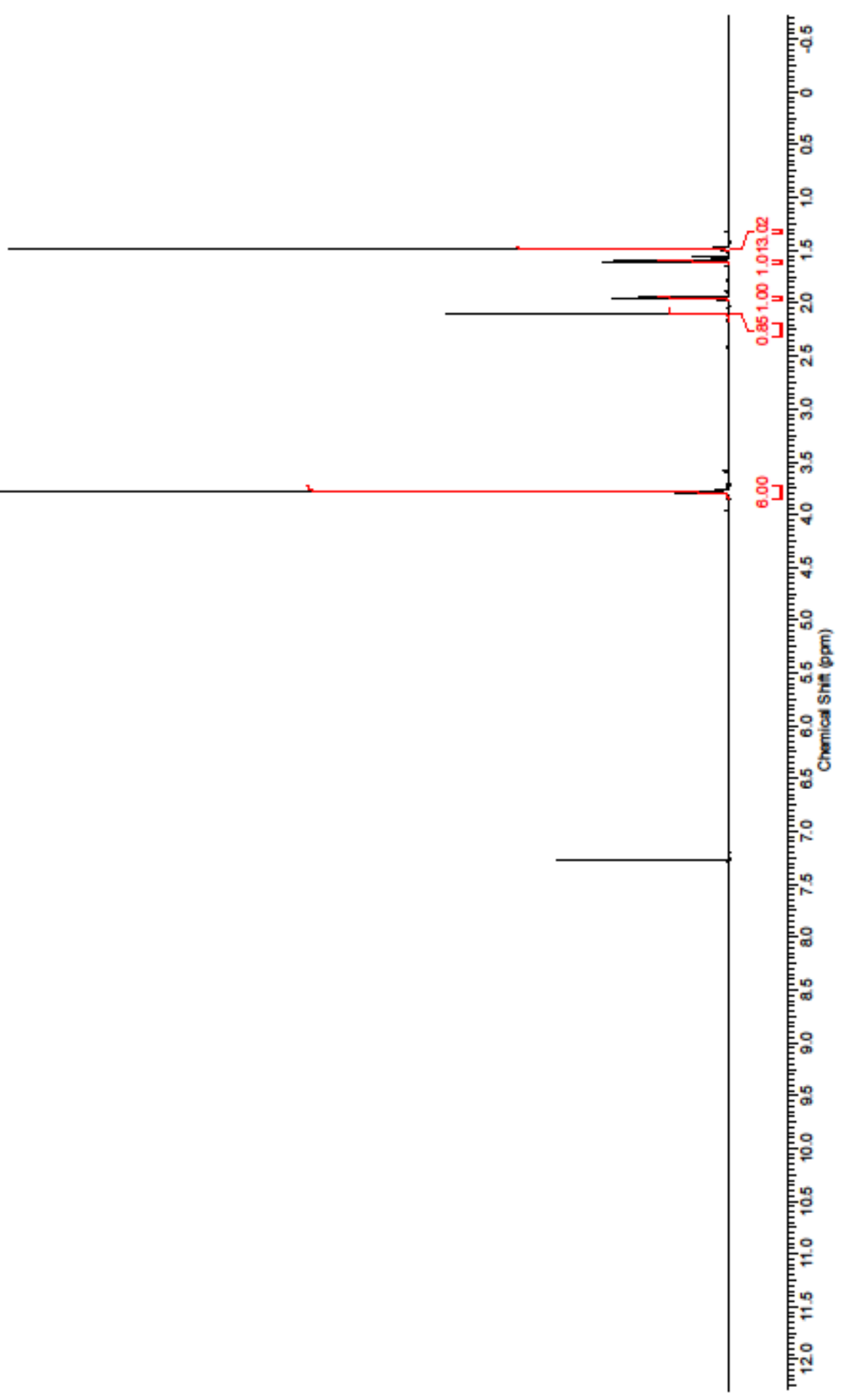




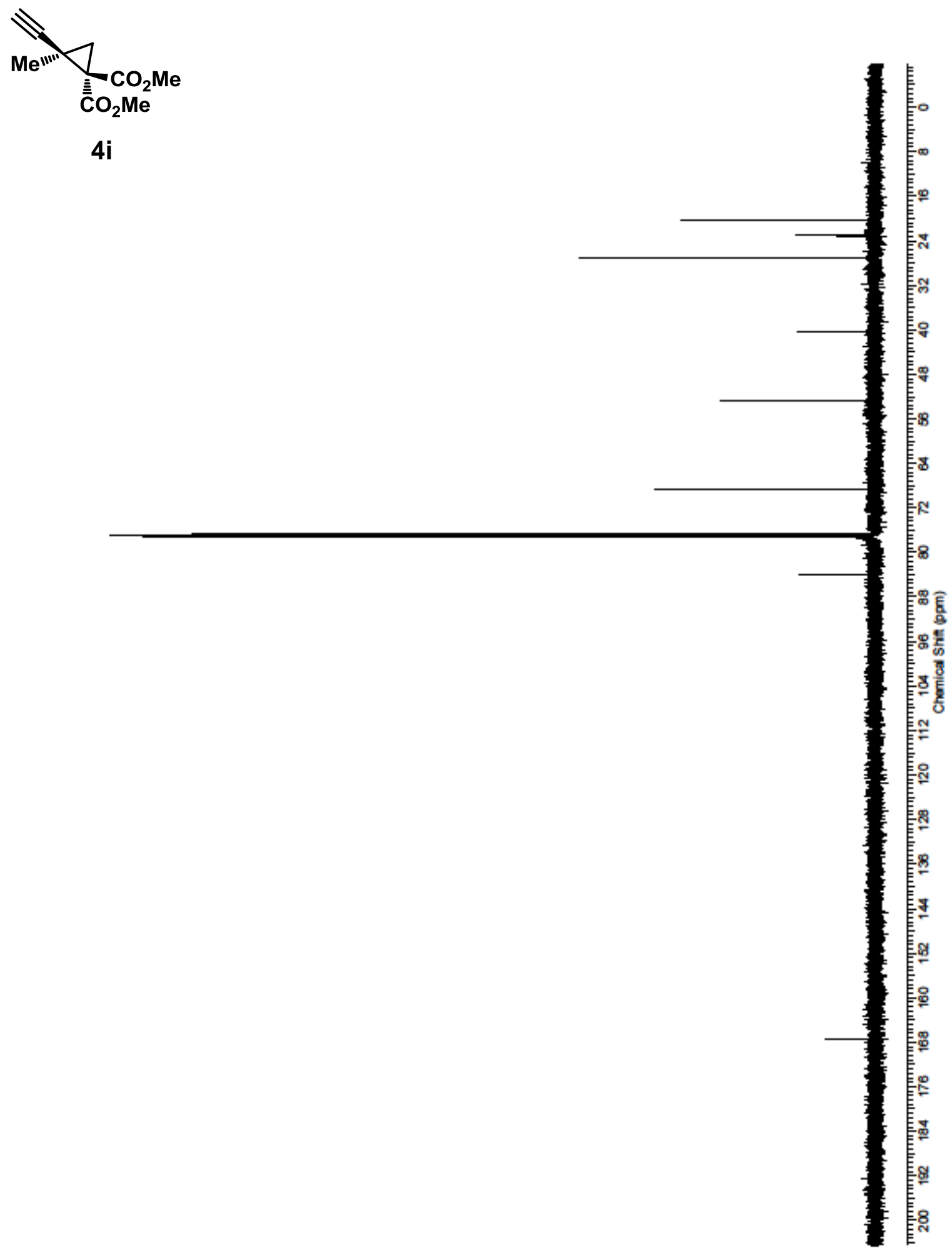

27 


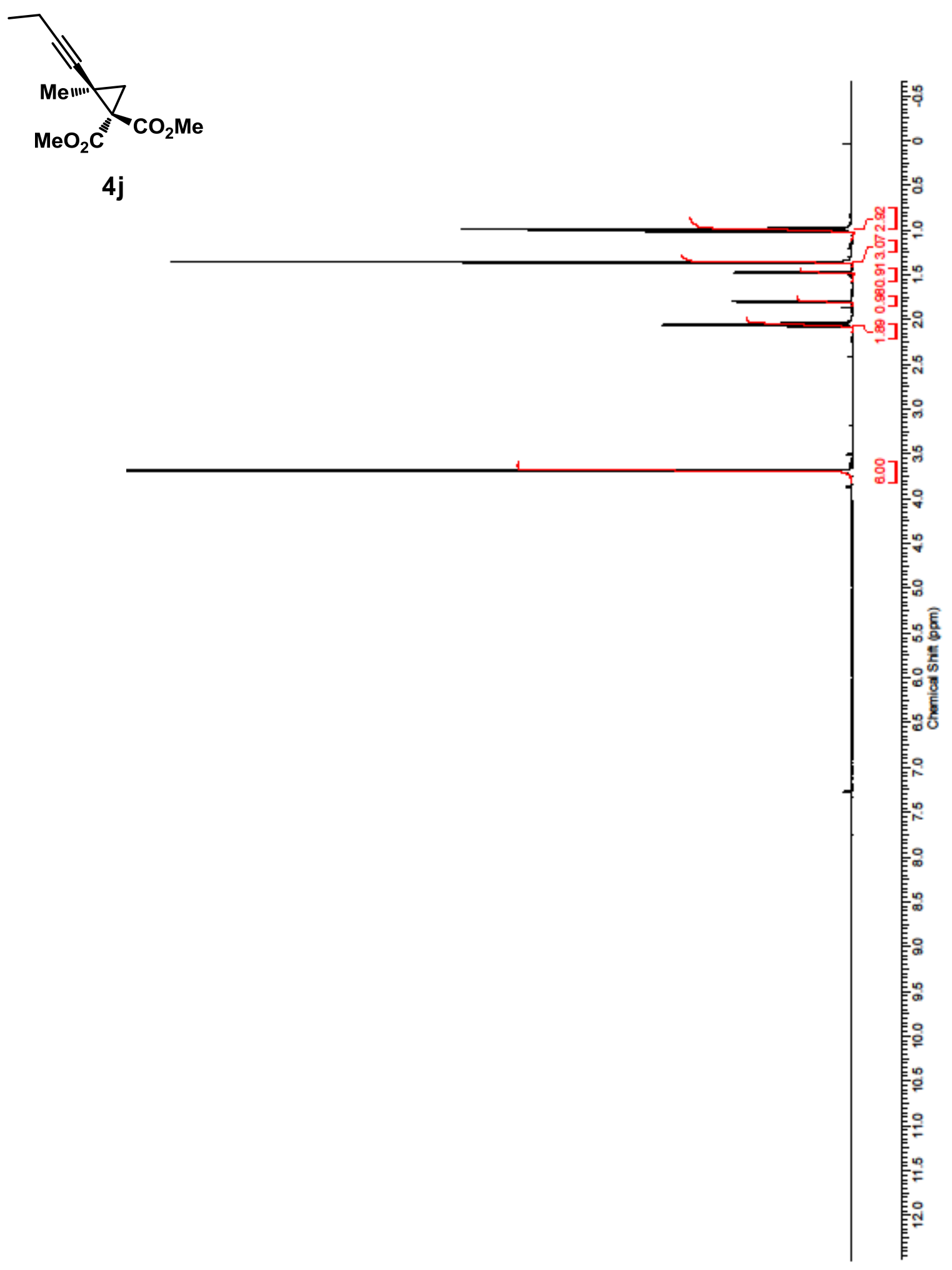




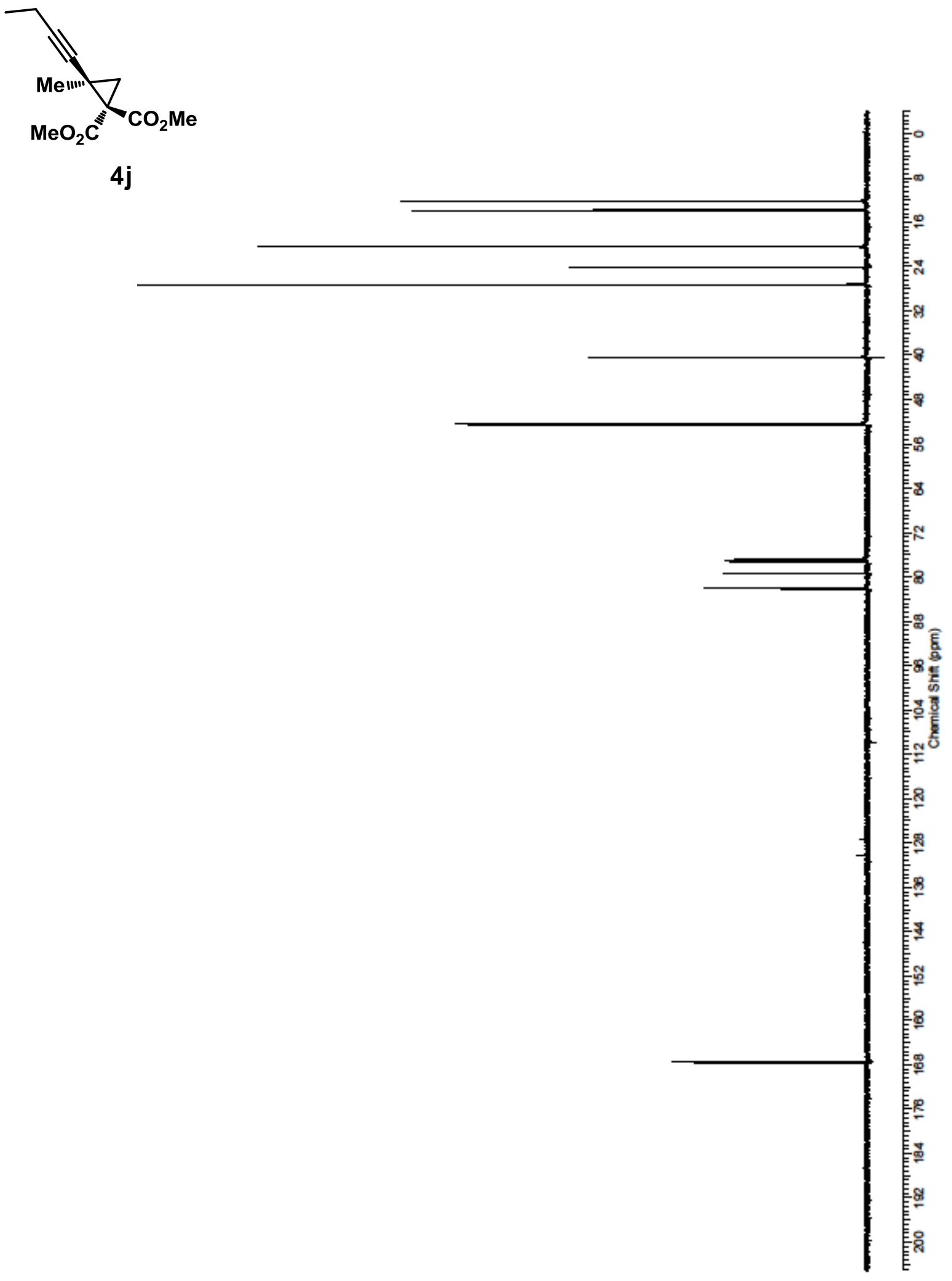



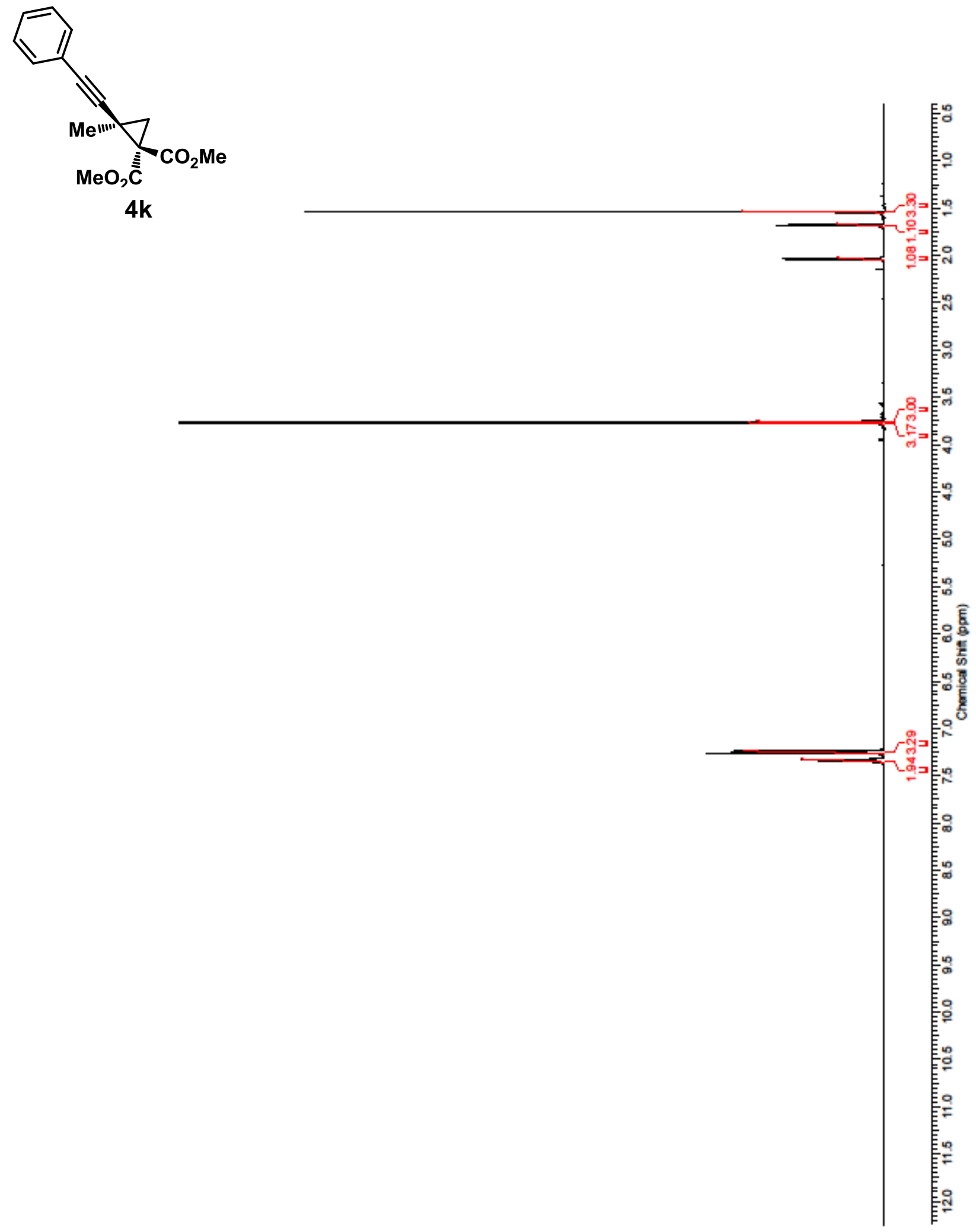

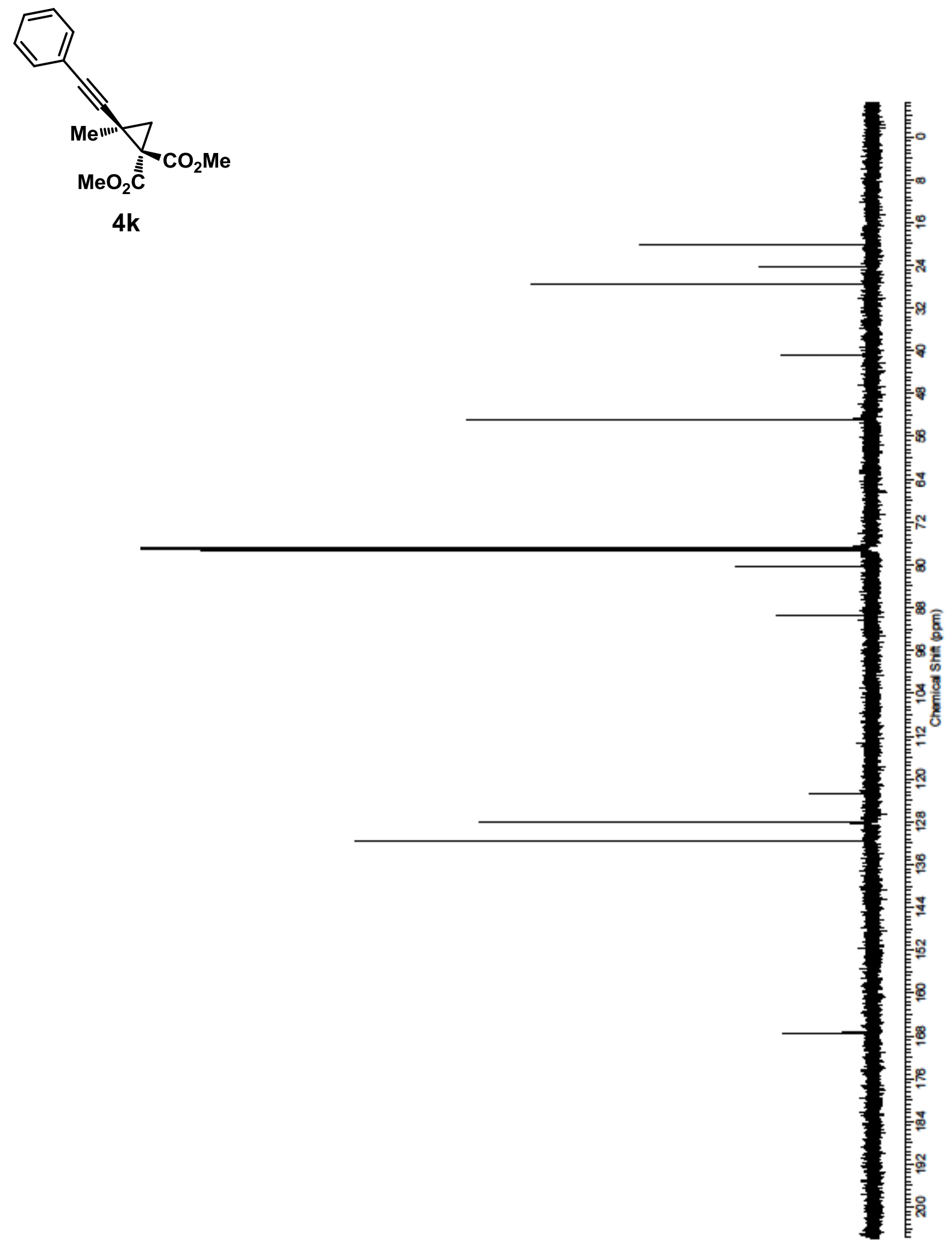

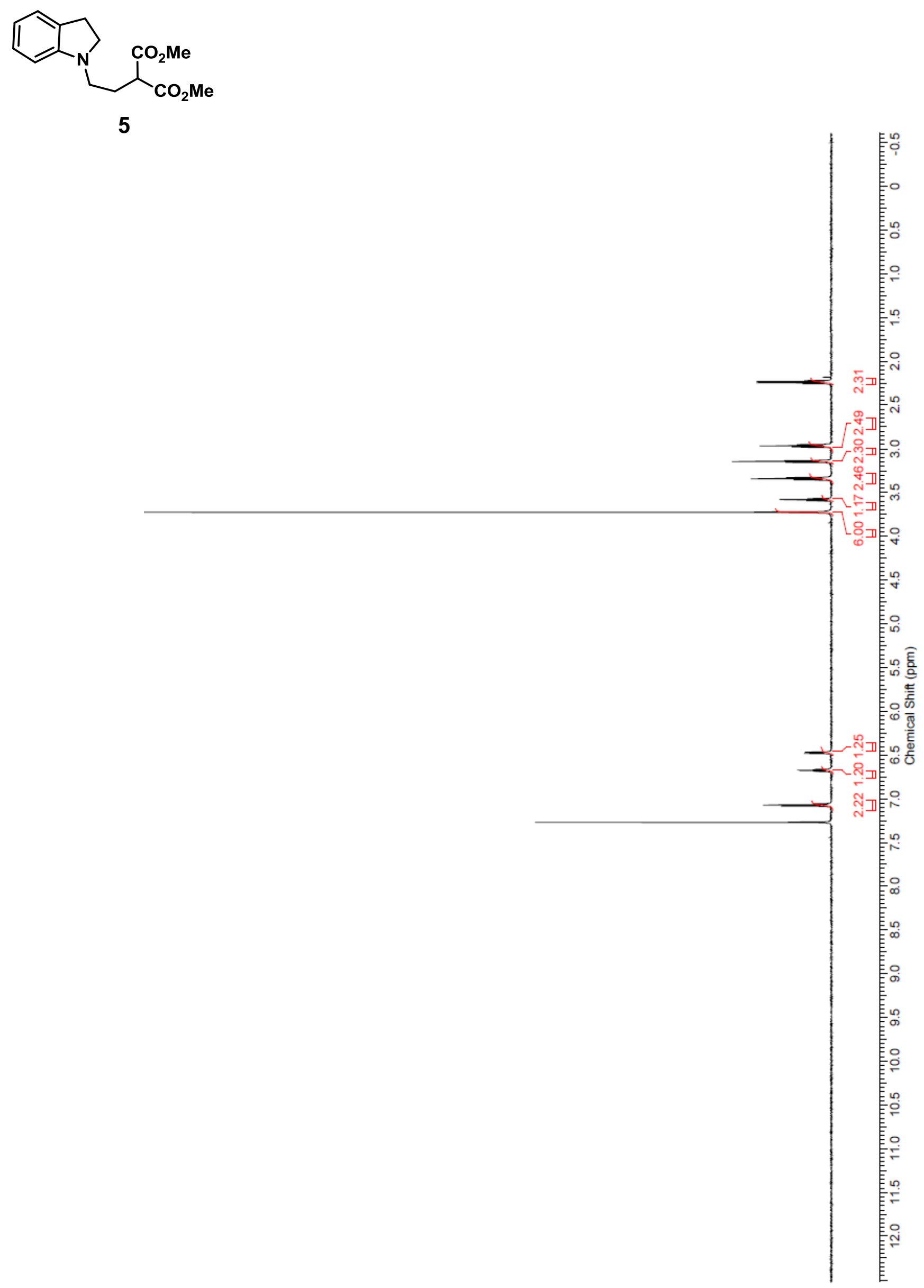


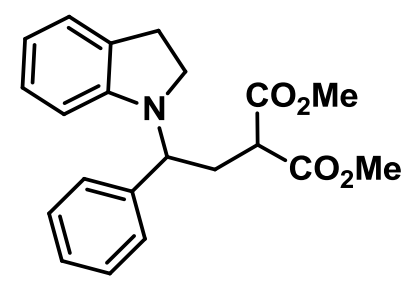

6

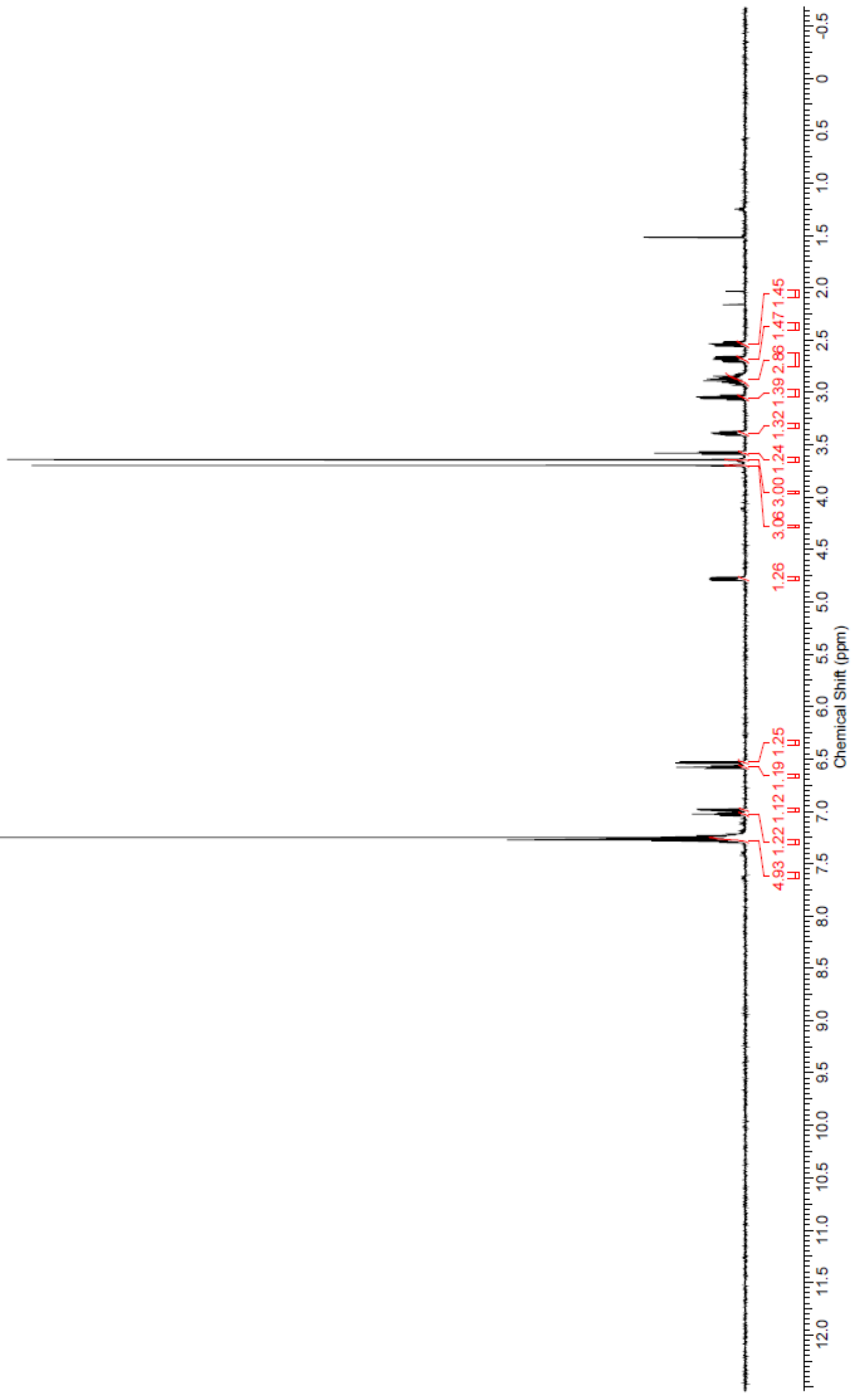



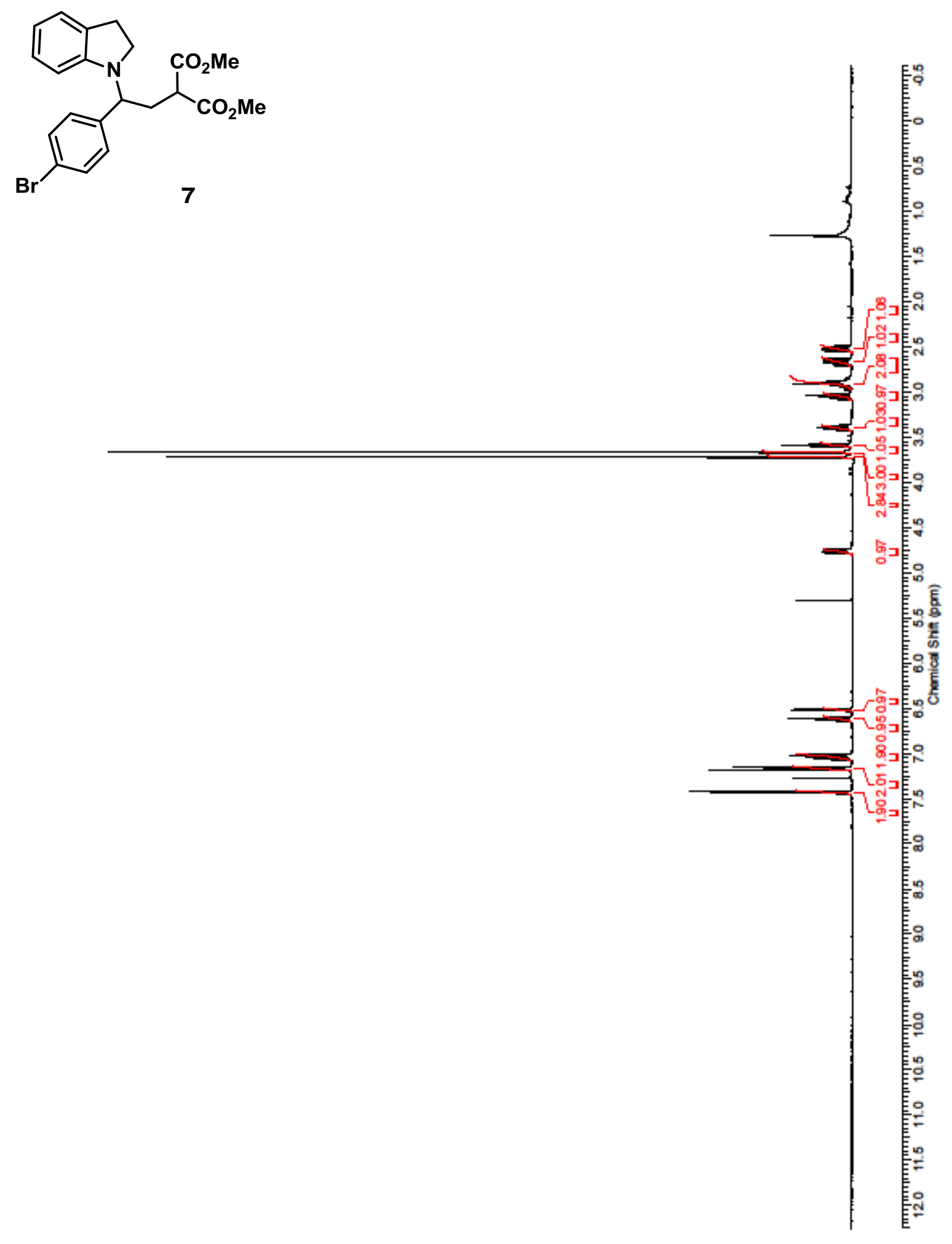

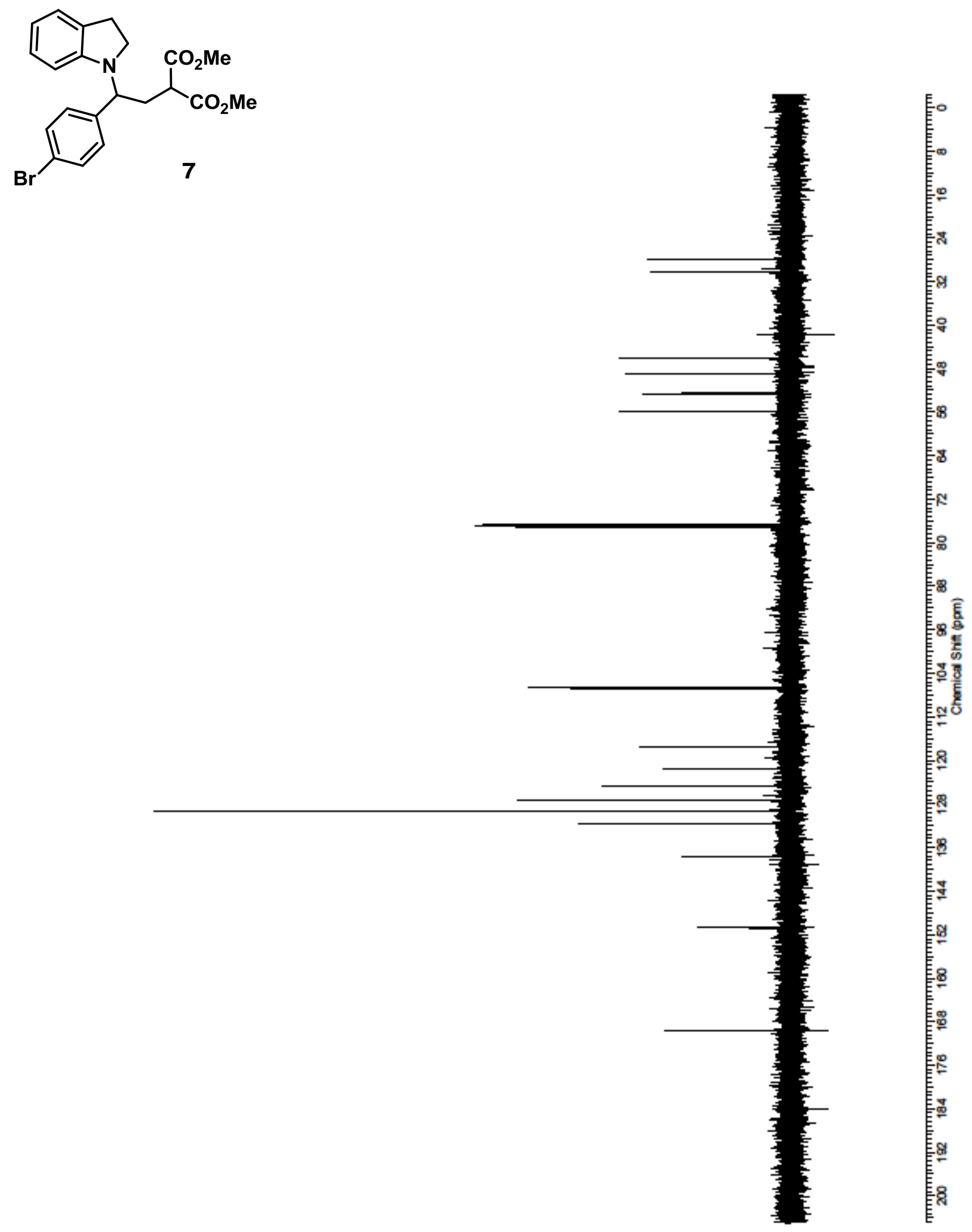

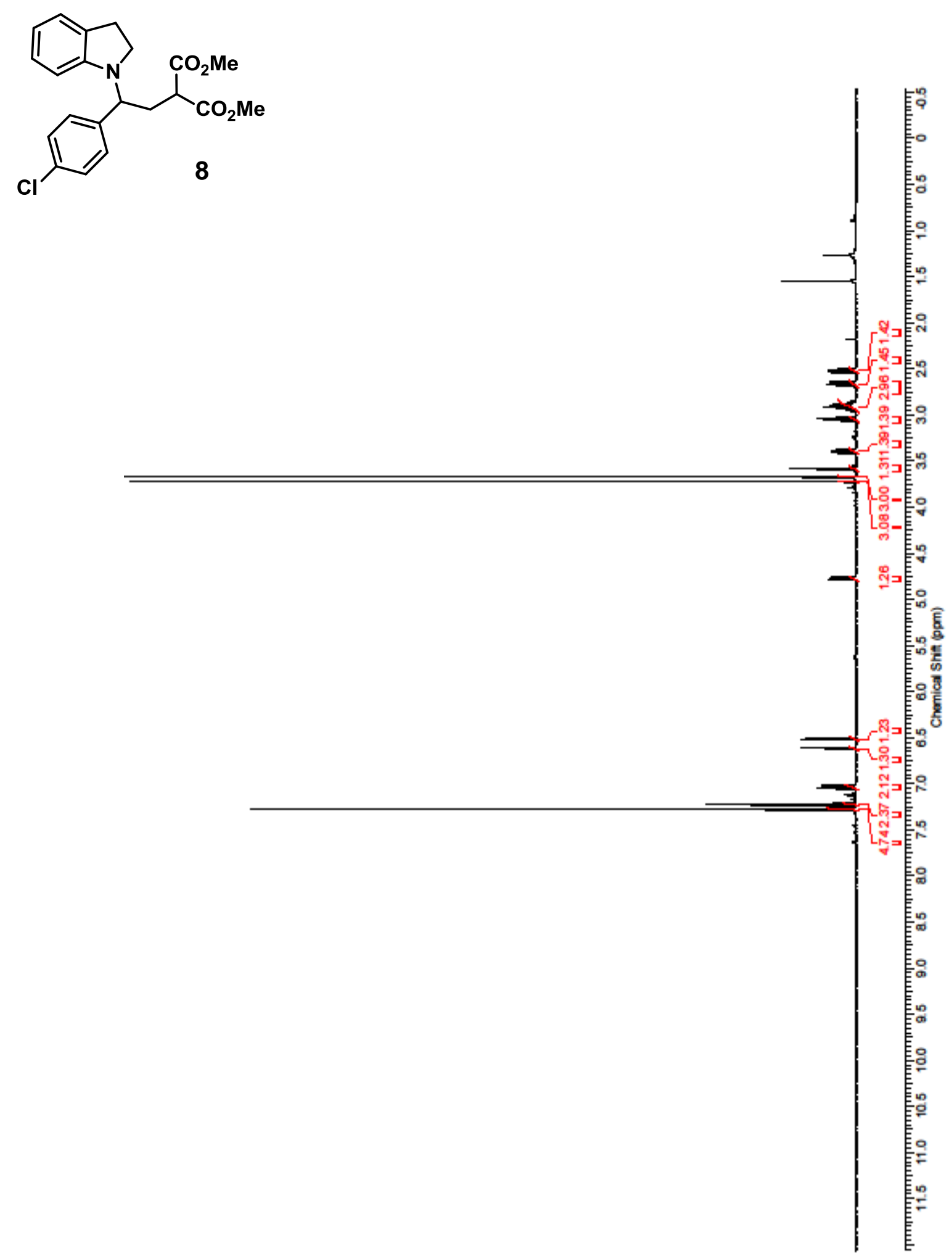
<smiles>COC(=O)C(CC(c1ccc(Cl)cc1)N1CCc2ccccc21)C(C)=O</smiles>

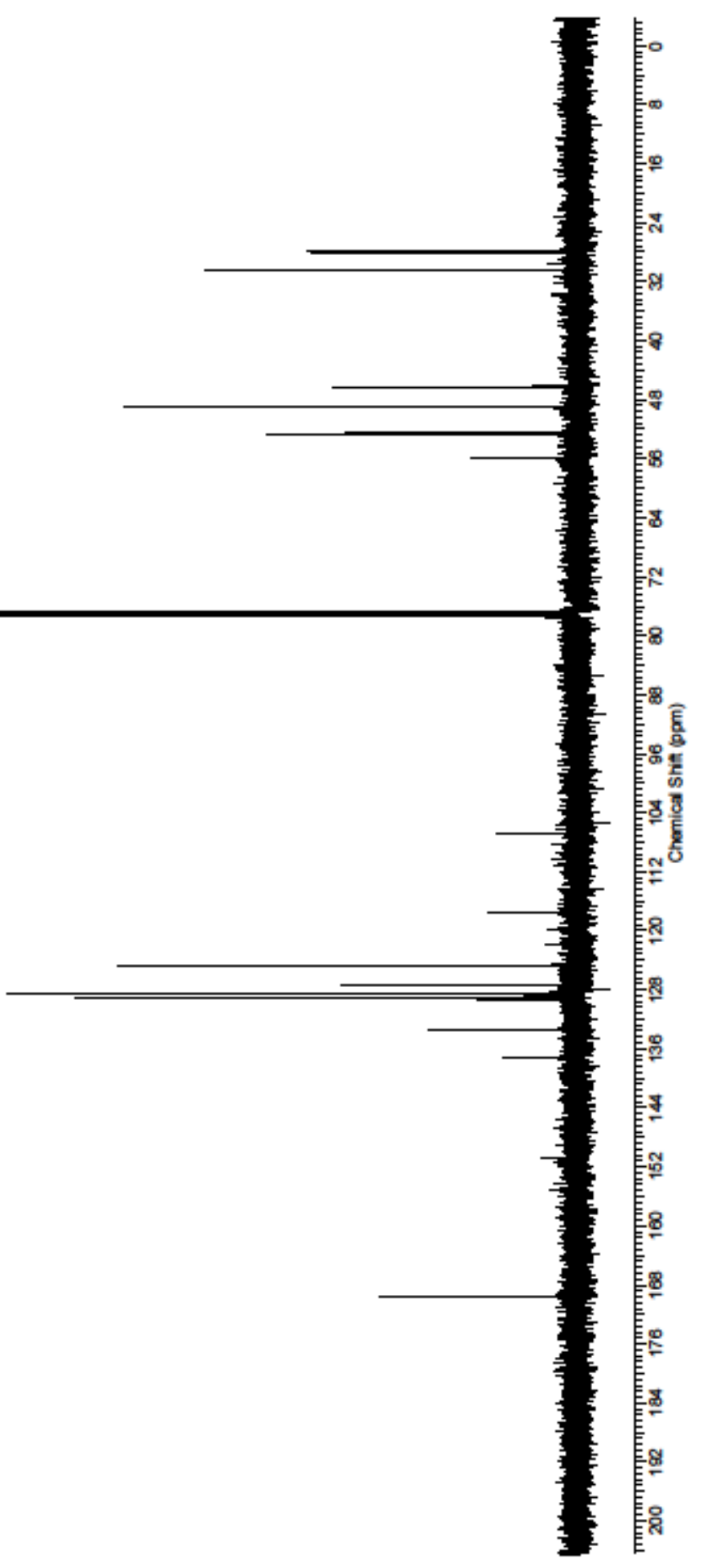



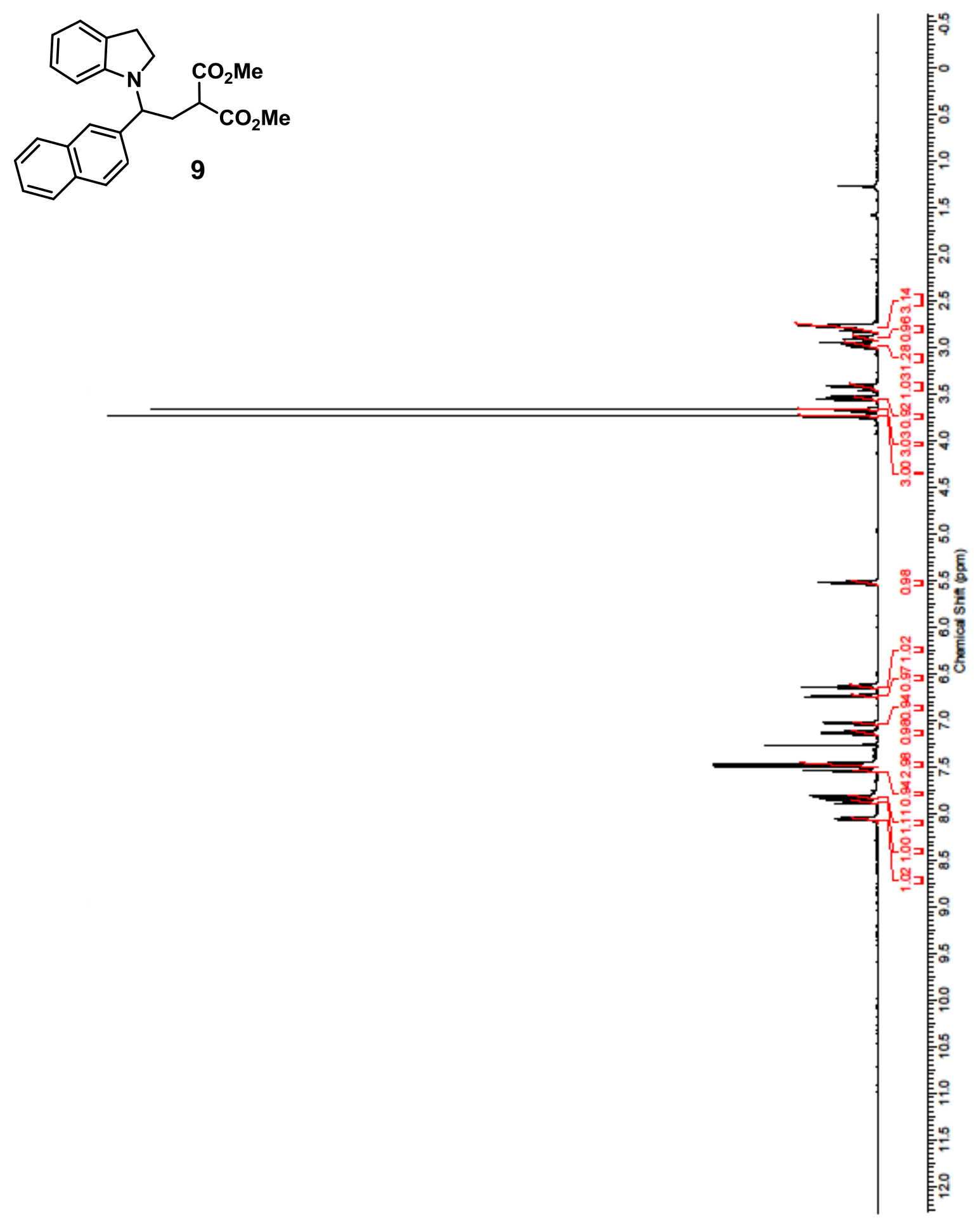
<smiles>COC(=O)C(CC(C(C)=O)C(C)=O)N1CCc2ccccc21</smiles>

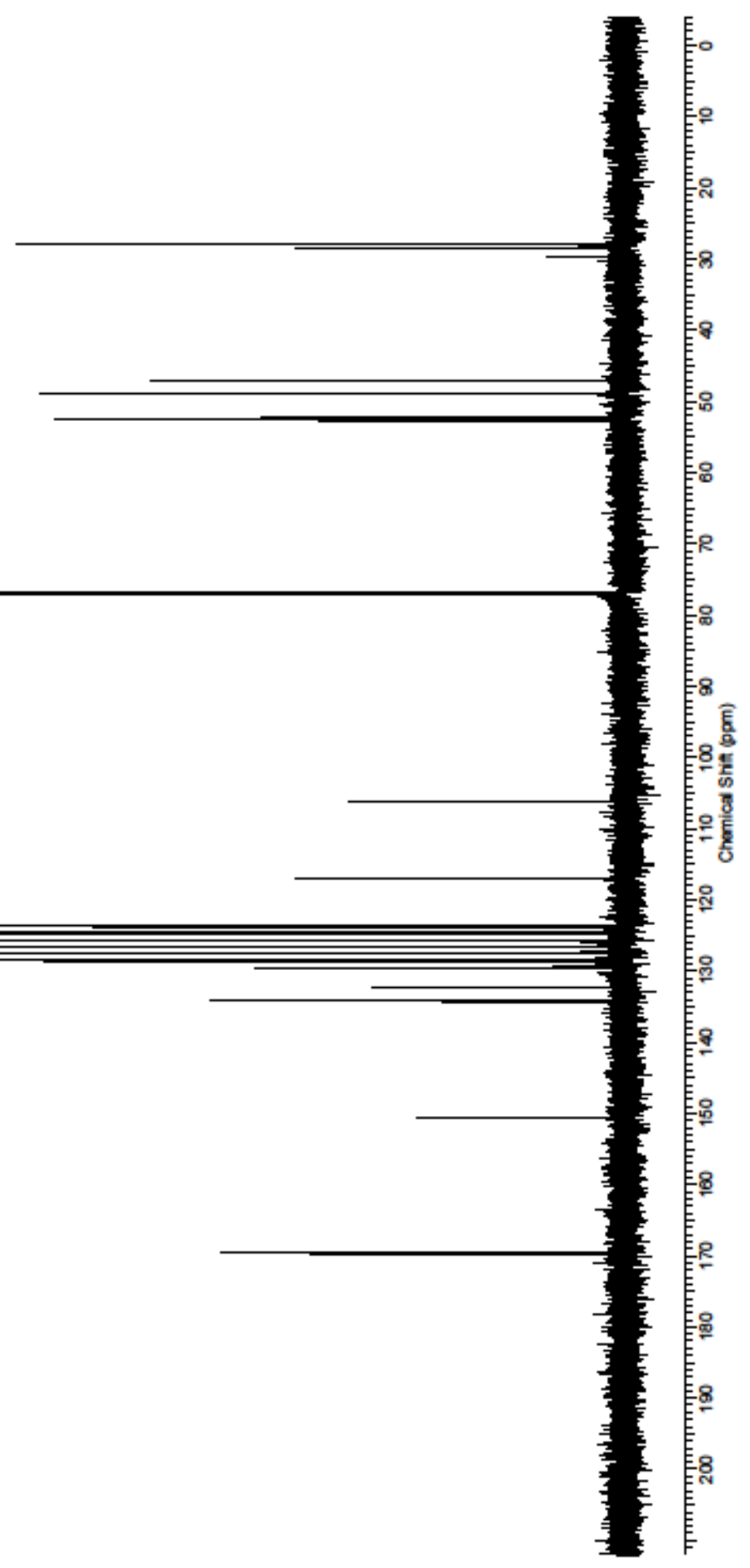




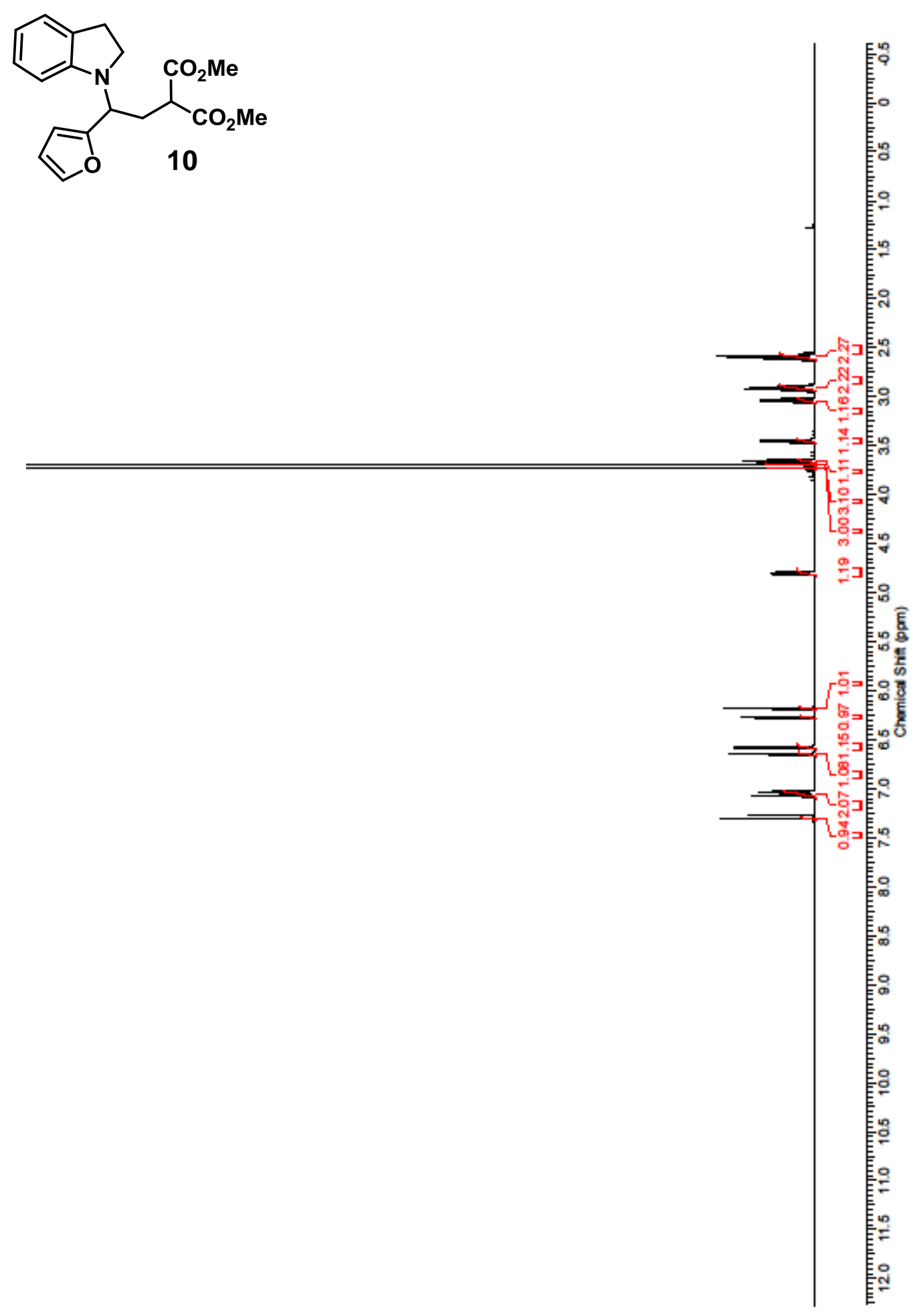




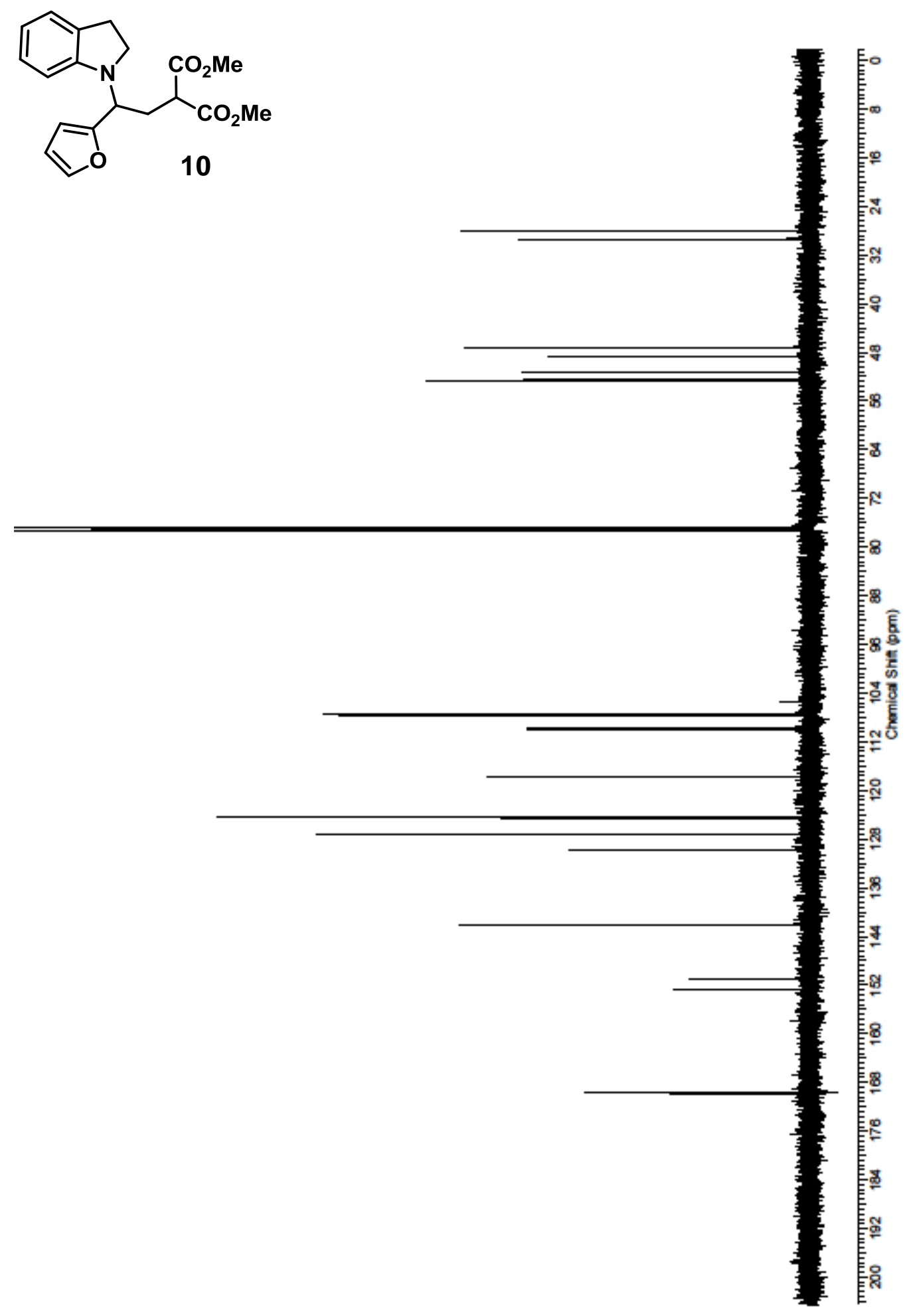



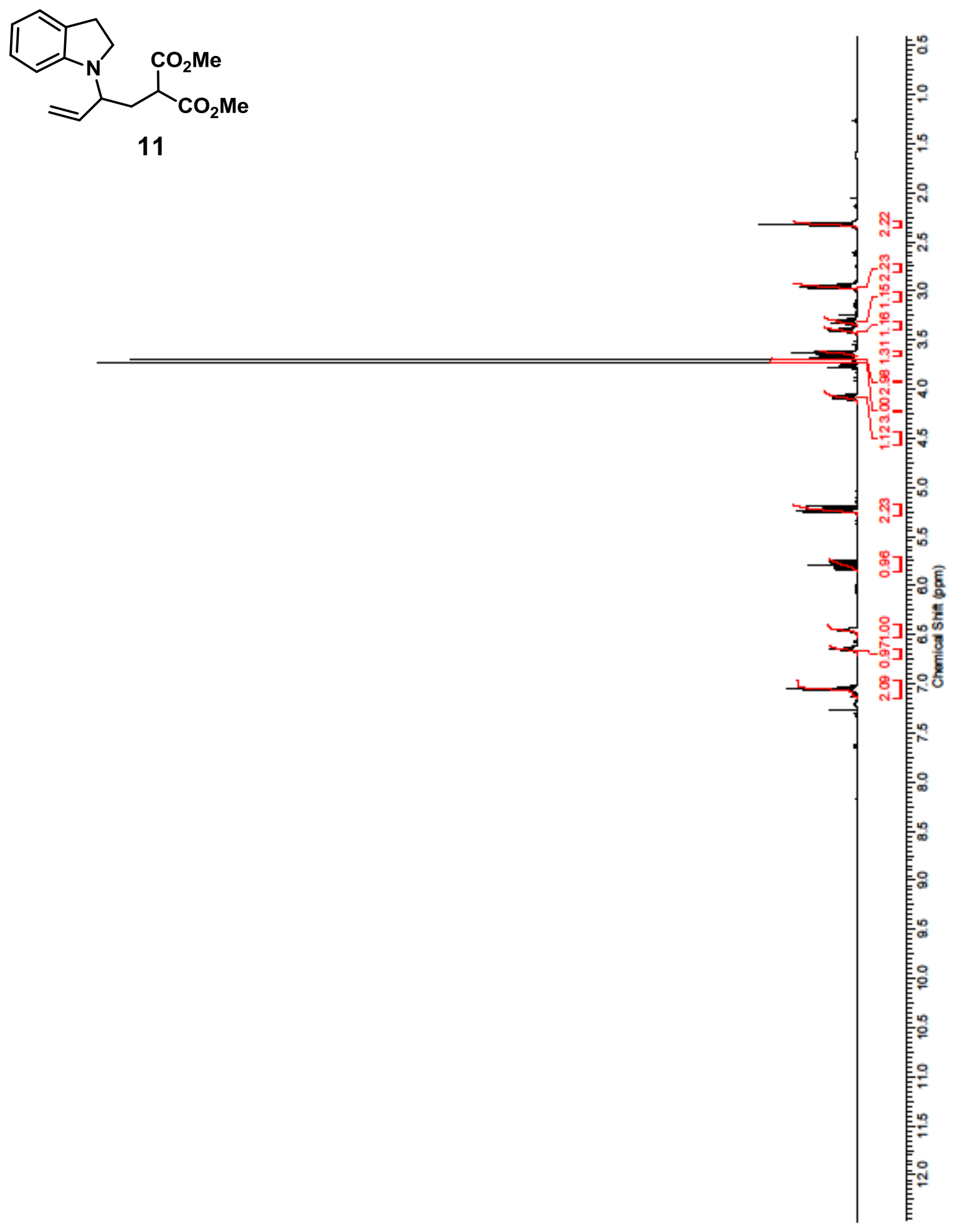


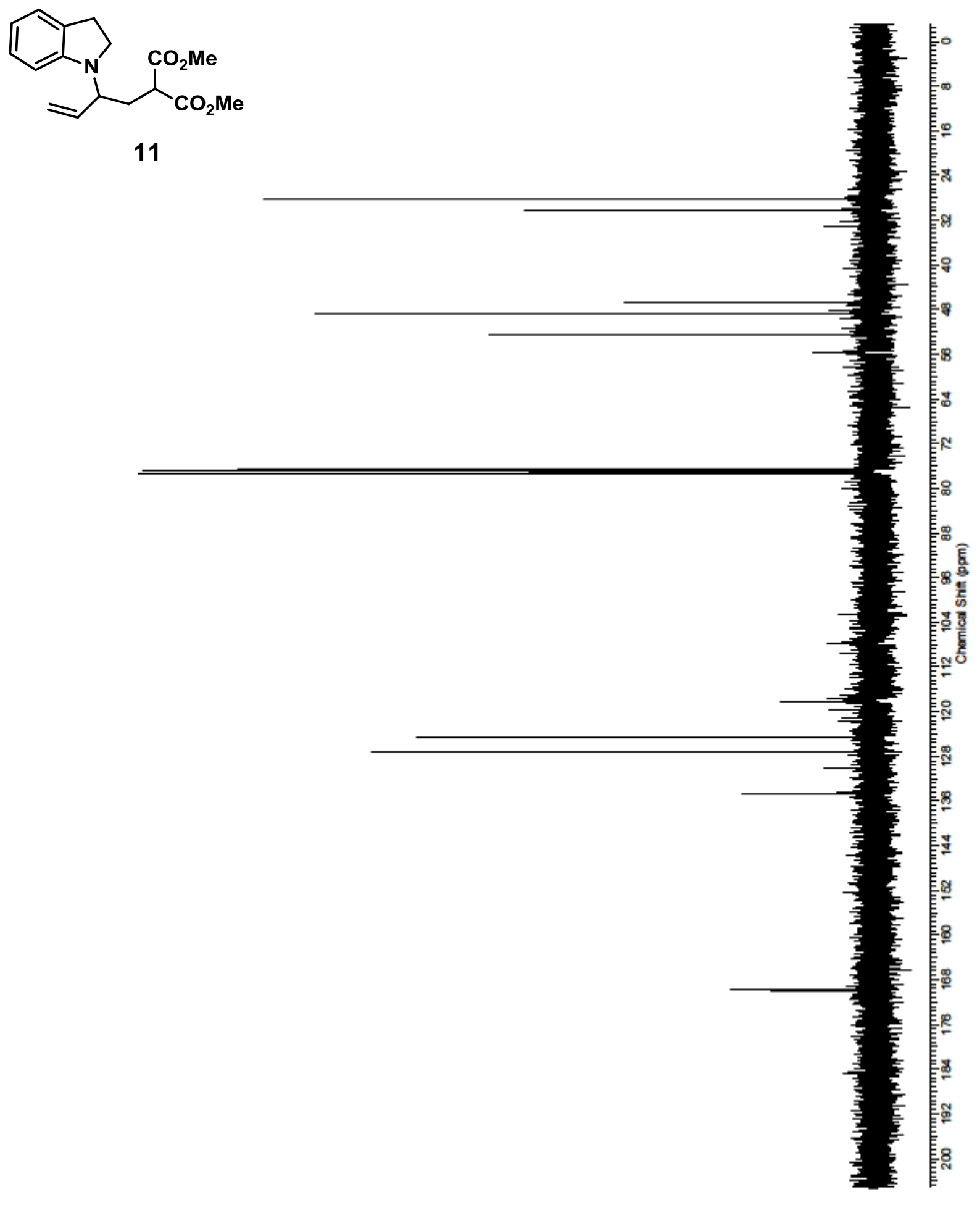




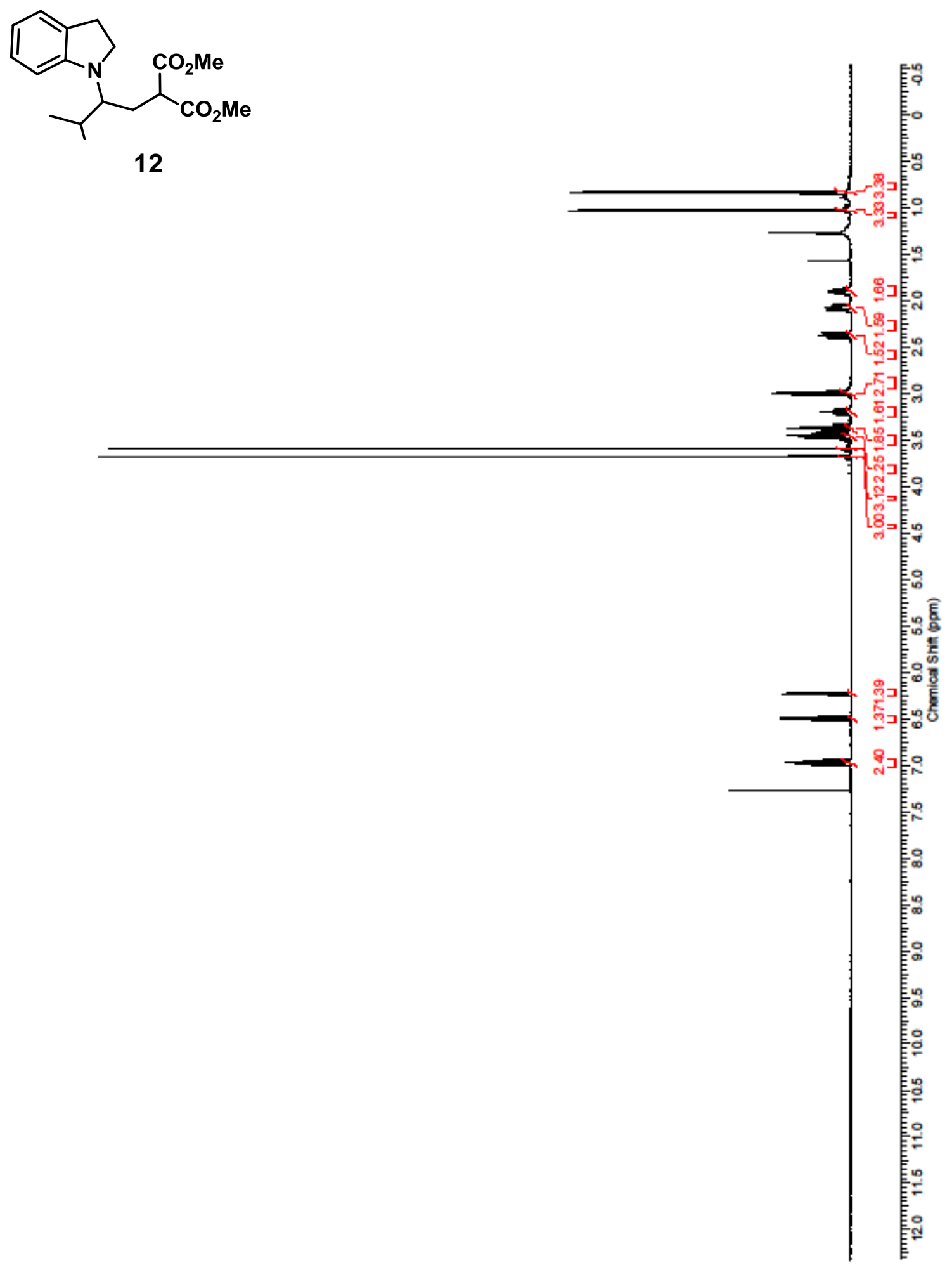



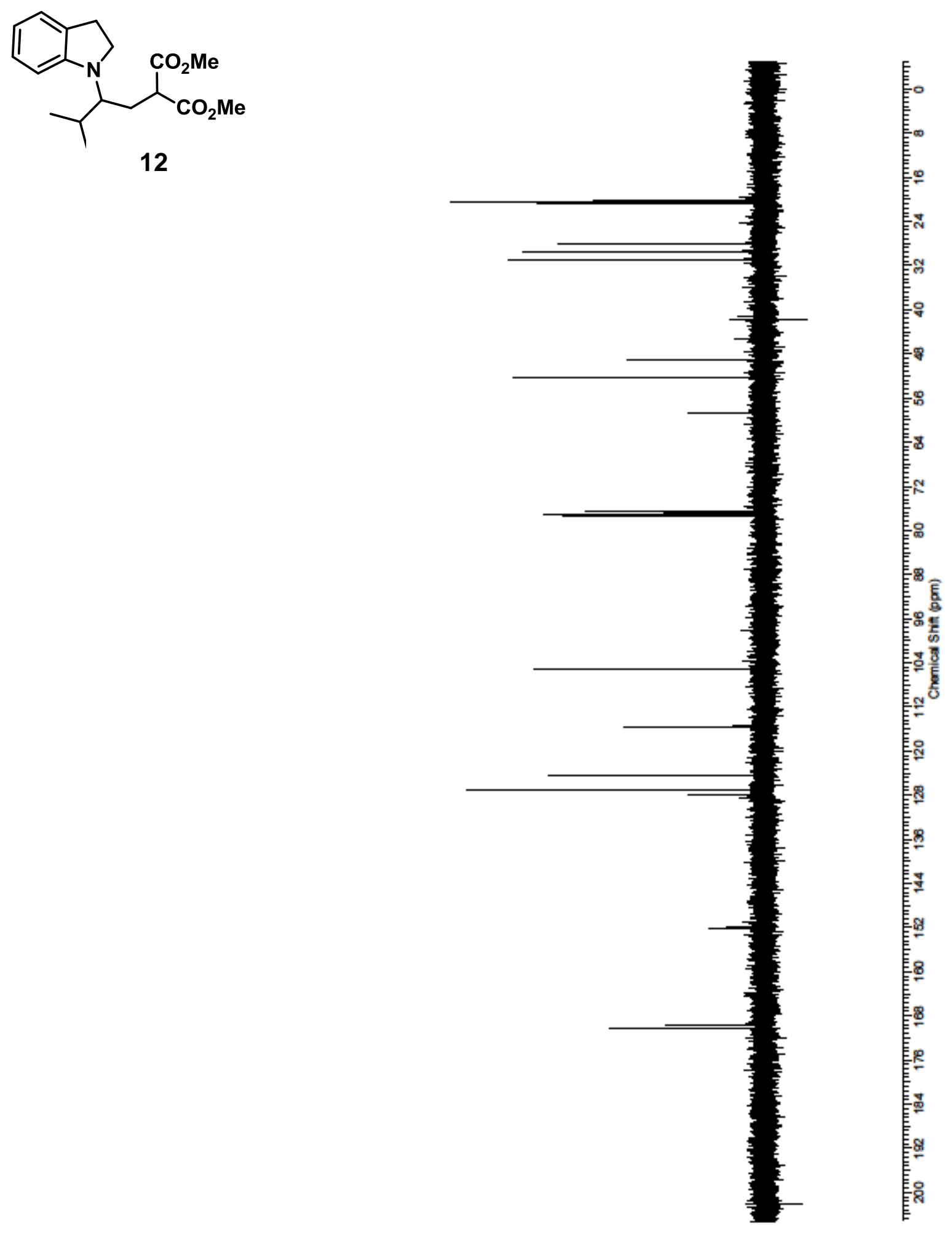


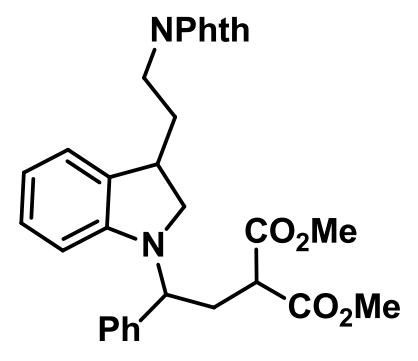

13

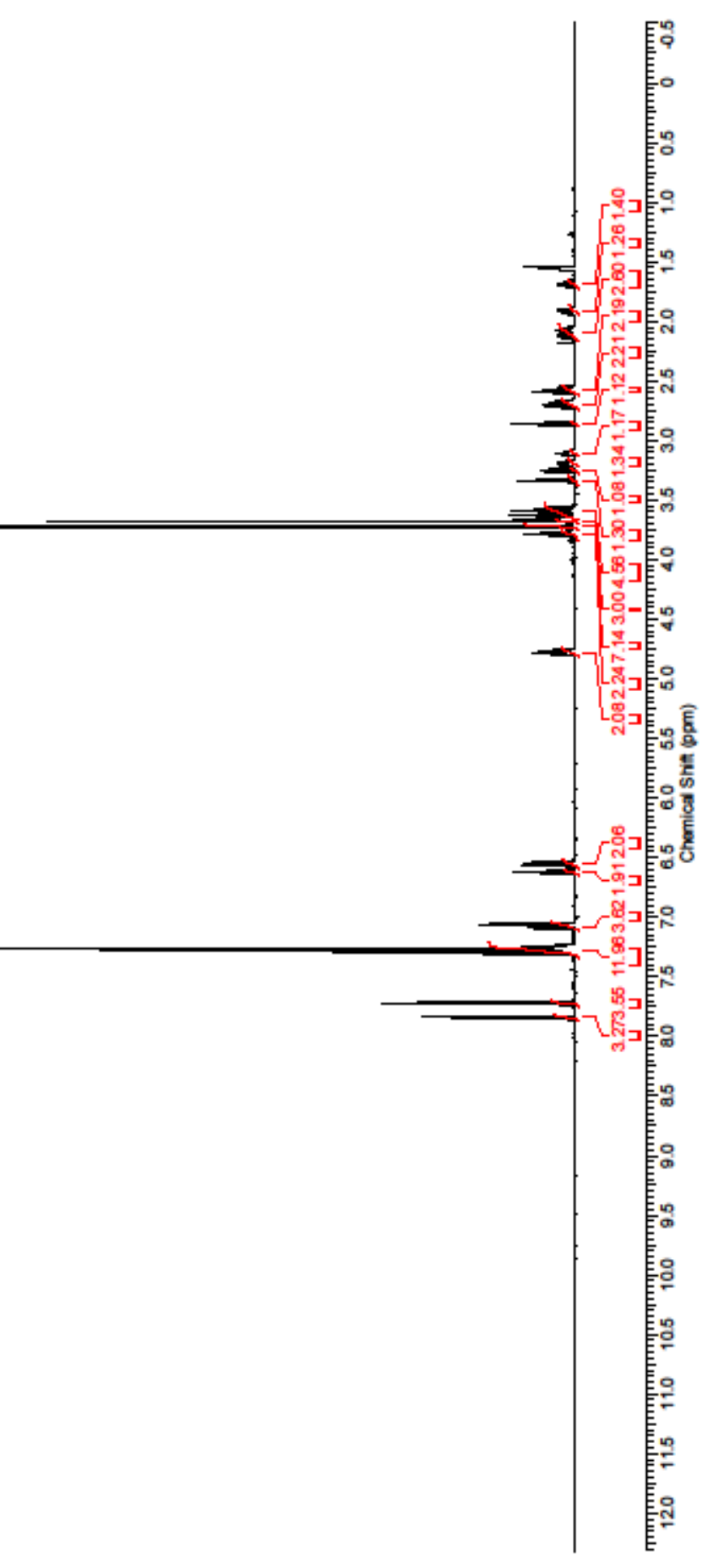




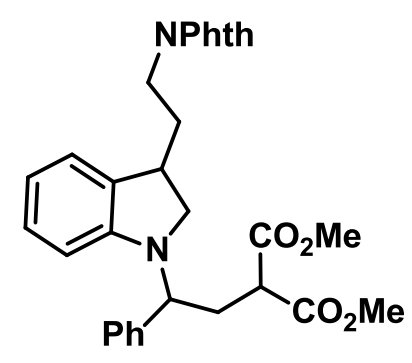

13

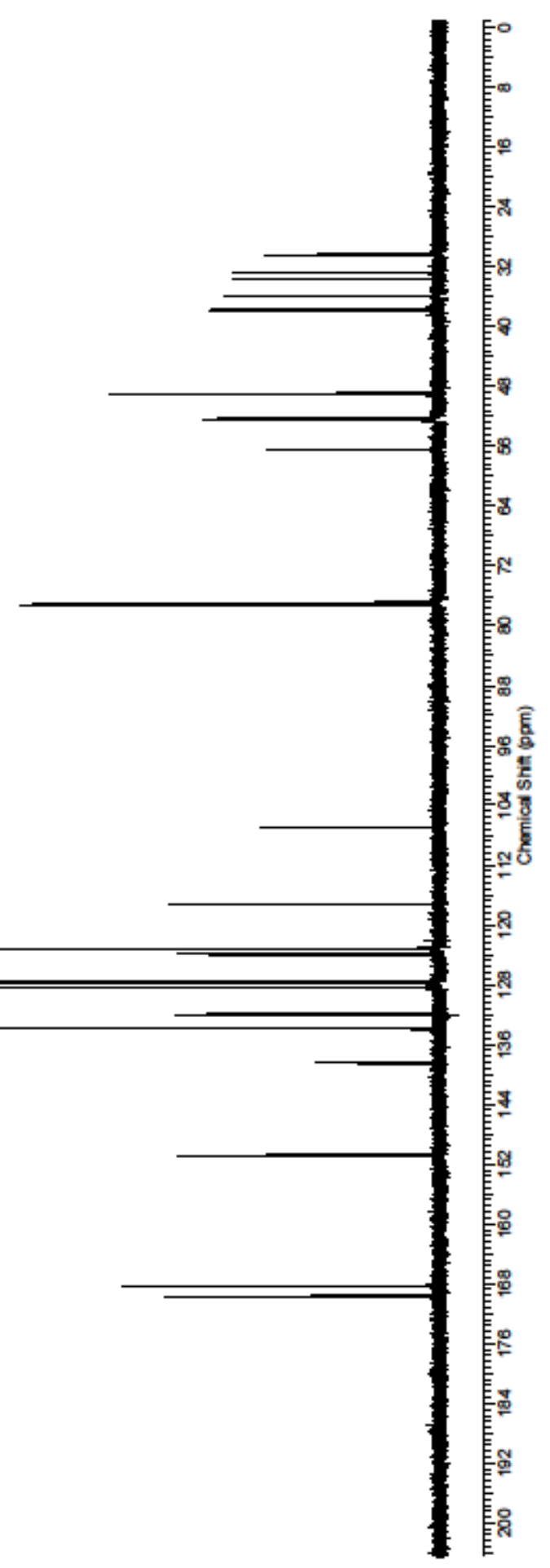




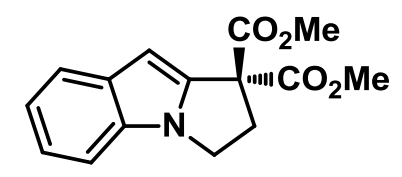

14

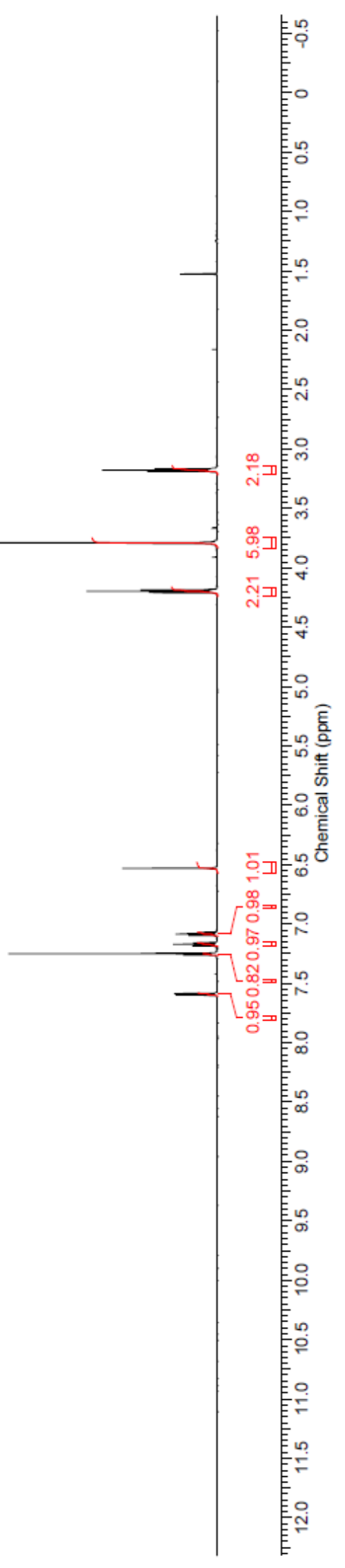




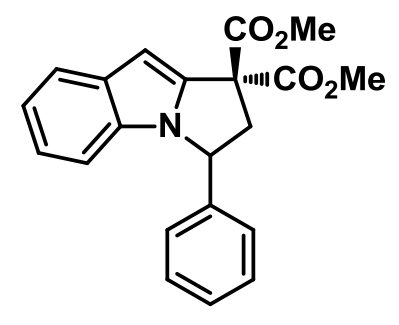

15

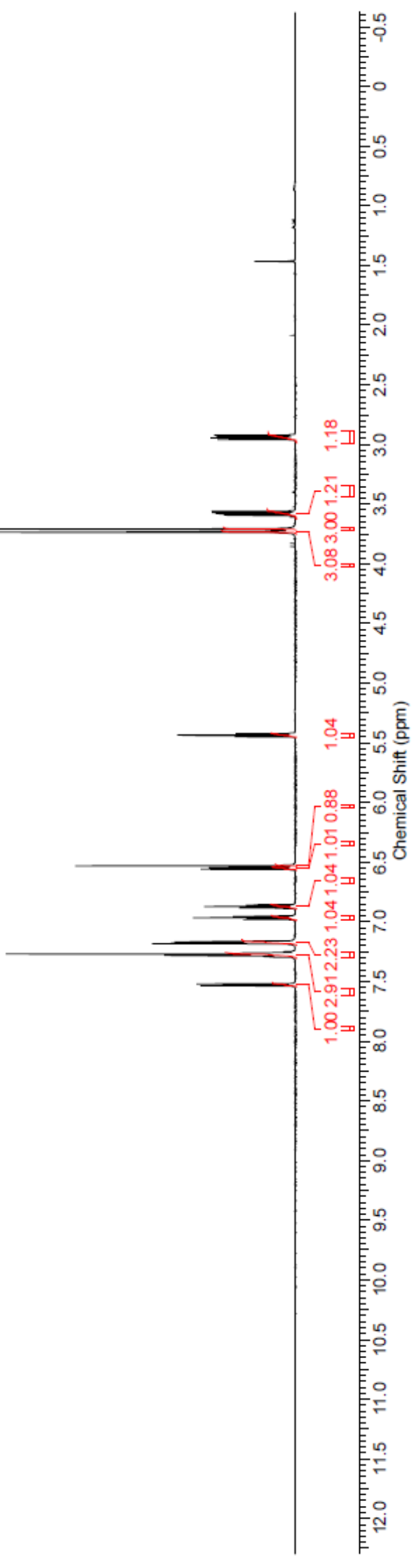




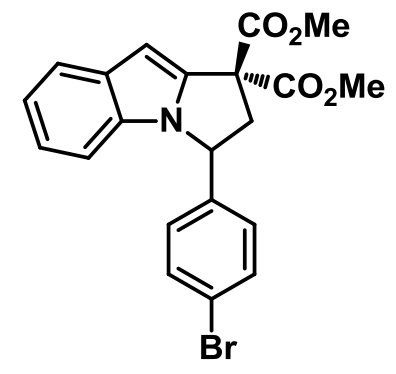

16

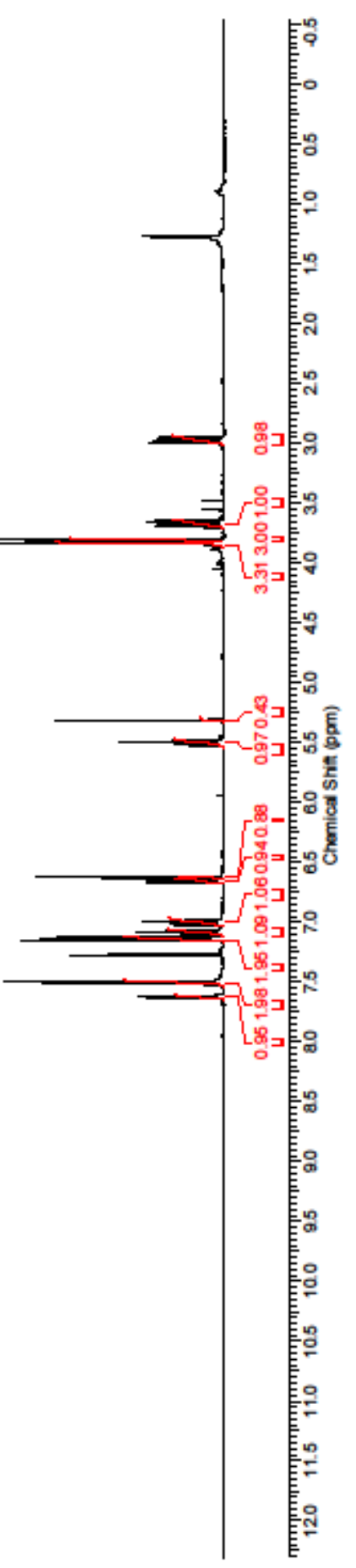



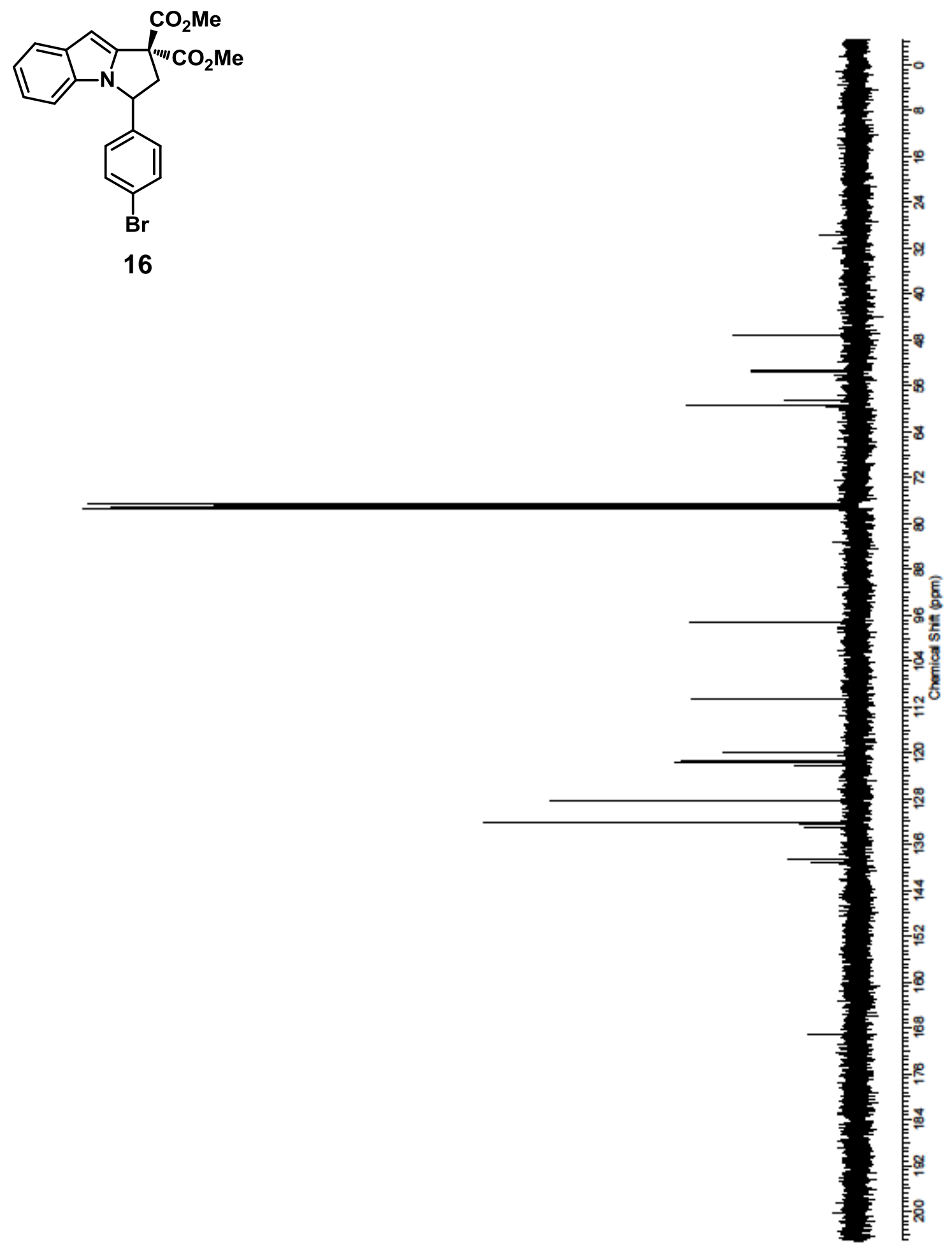

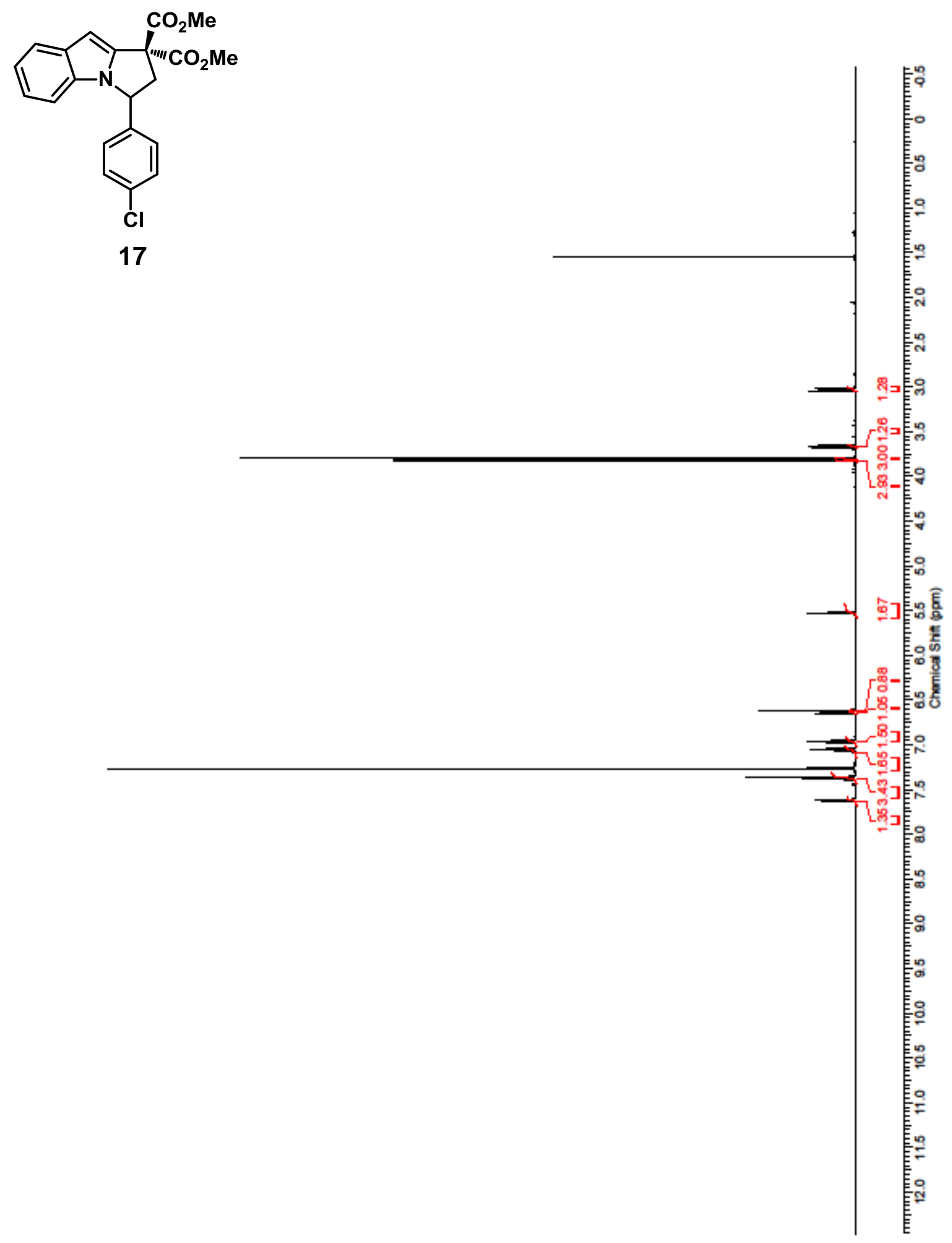

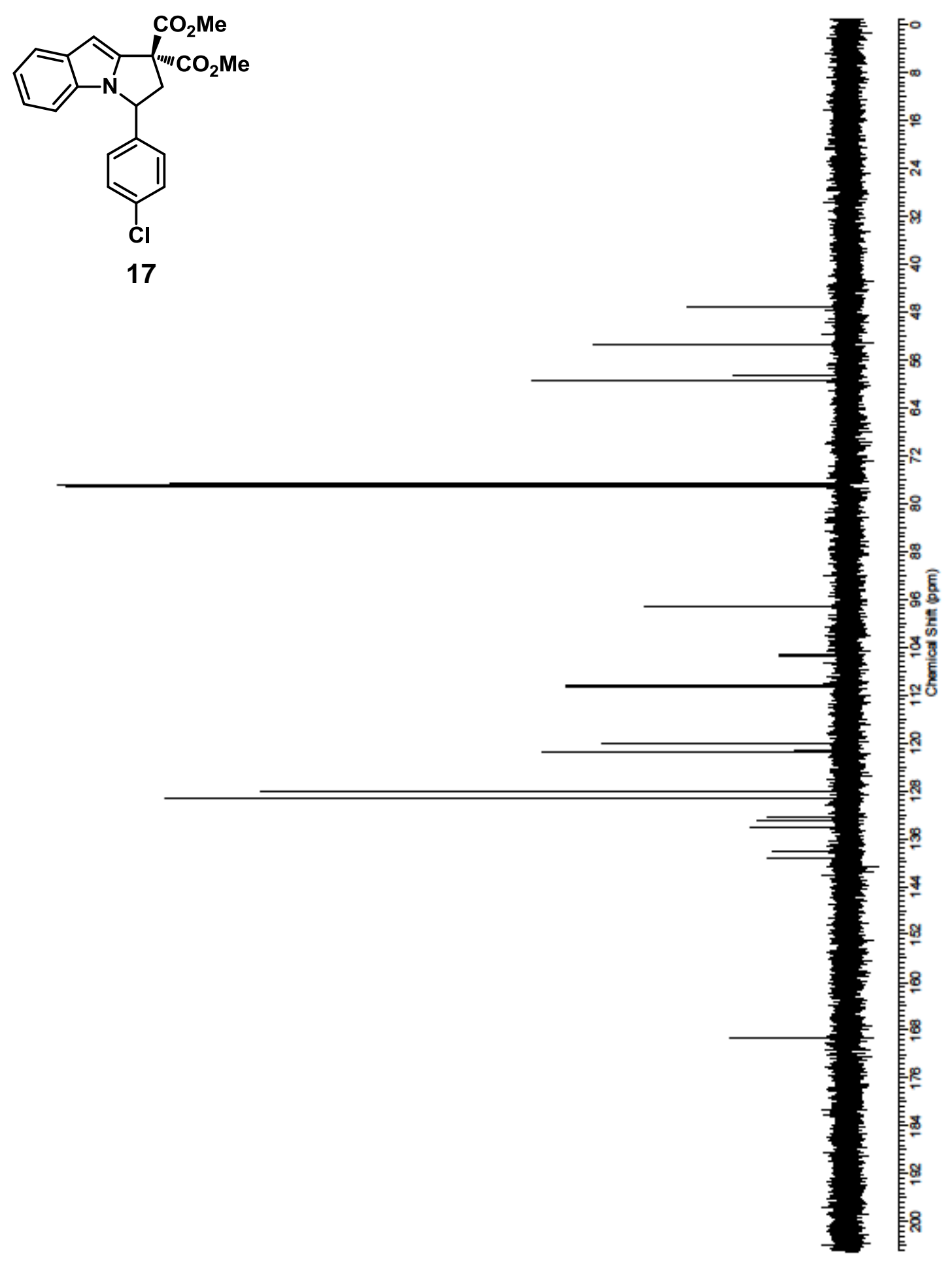


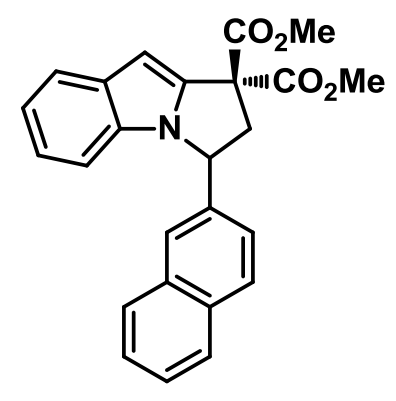

18

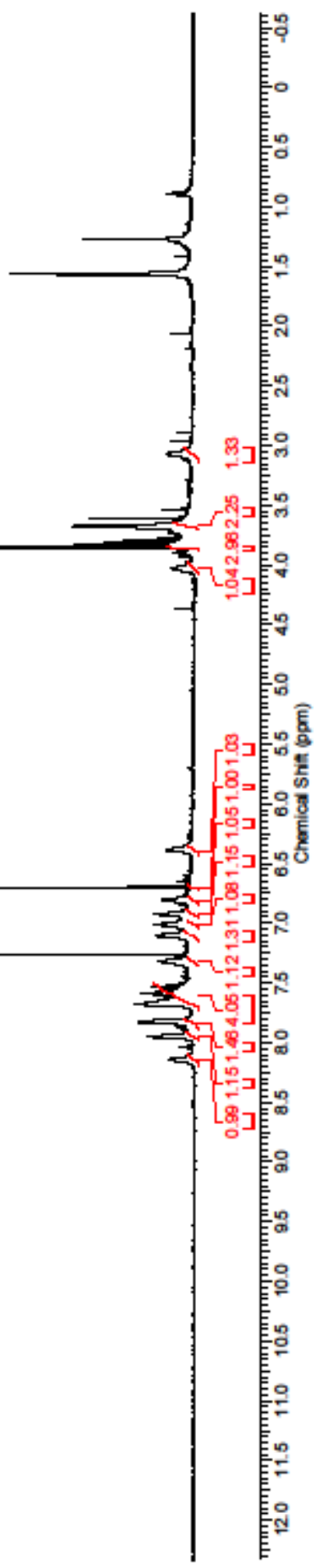




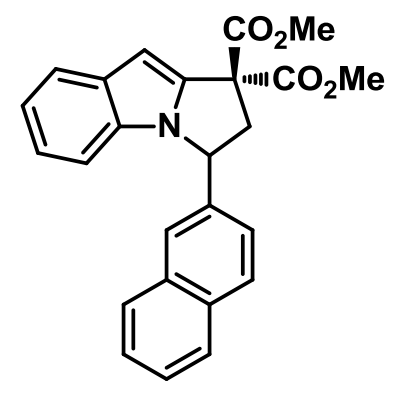

18

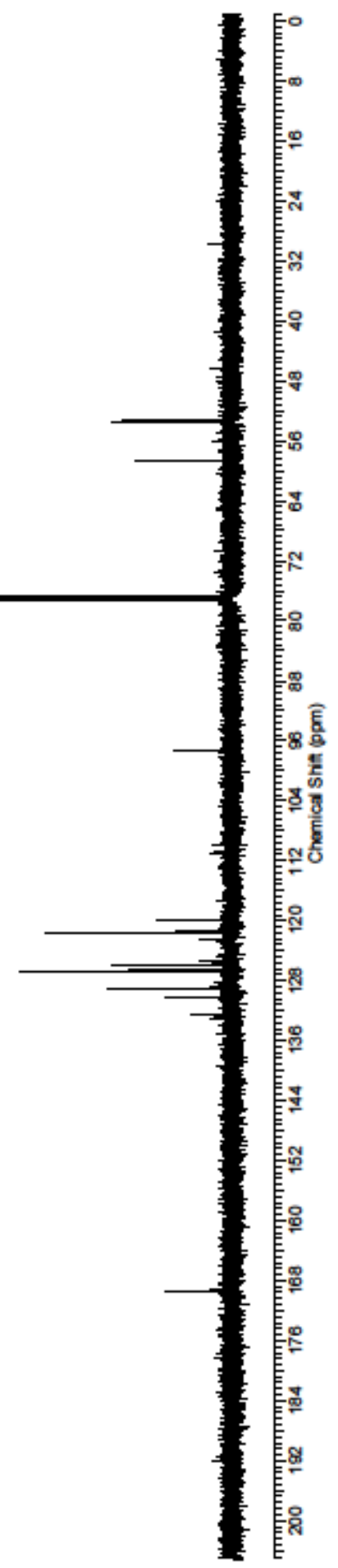




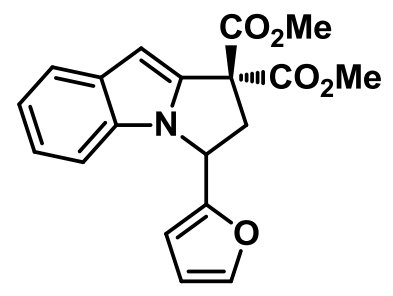

19

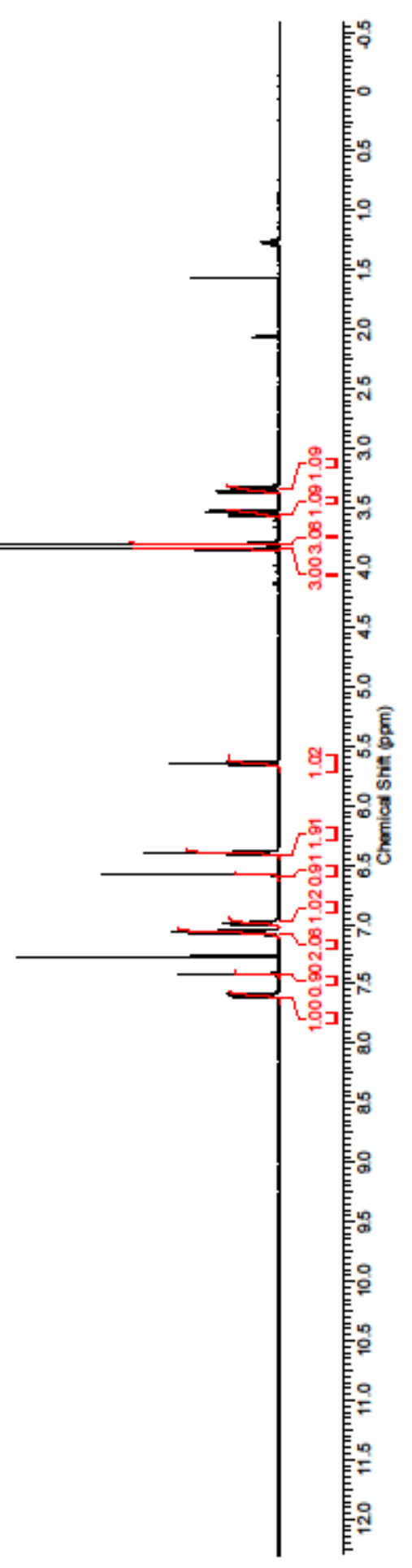




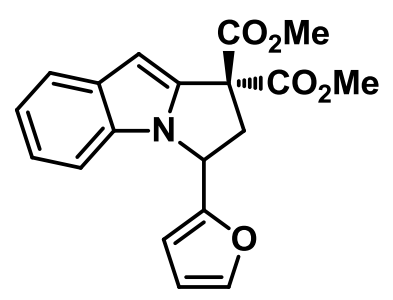

19

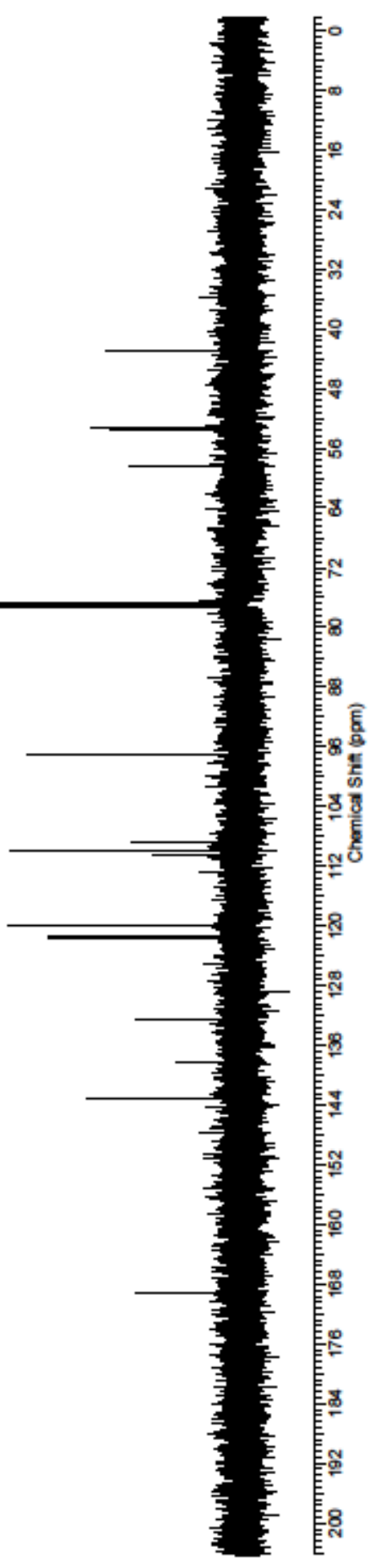



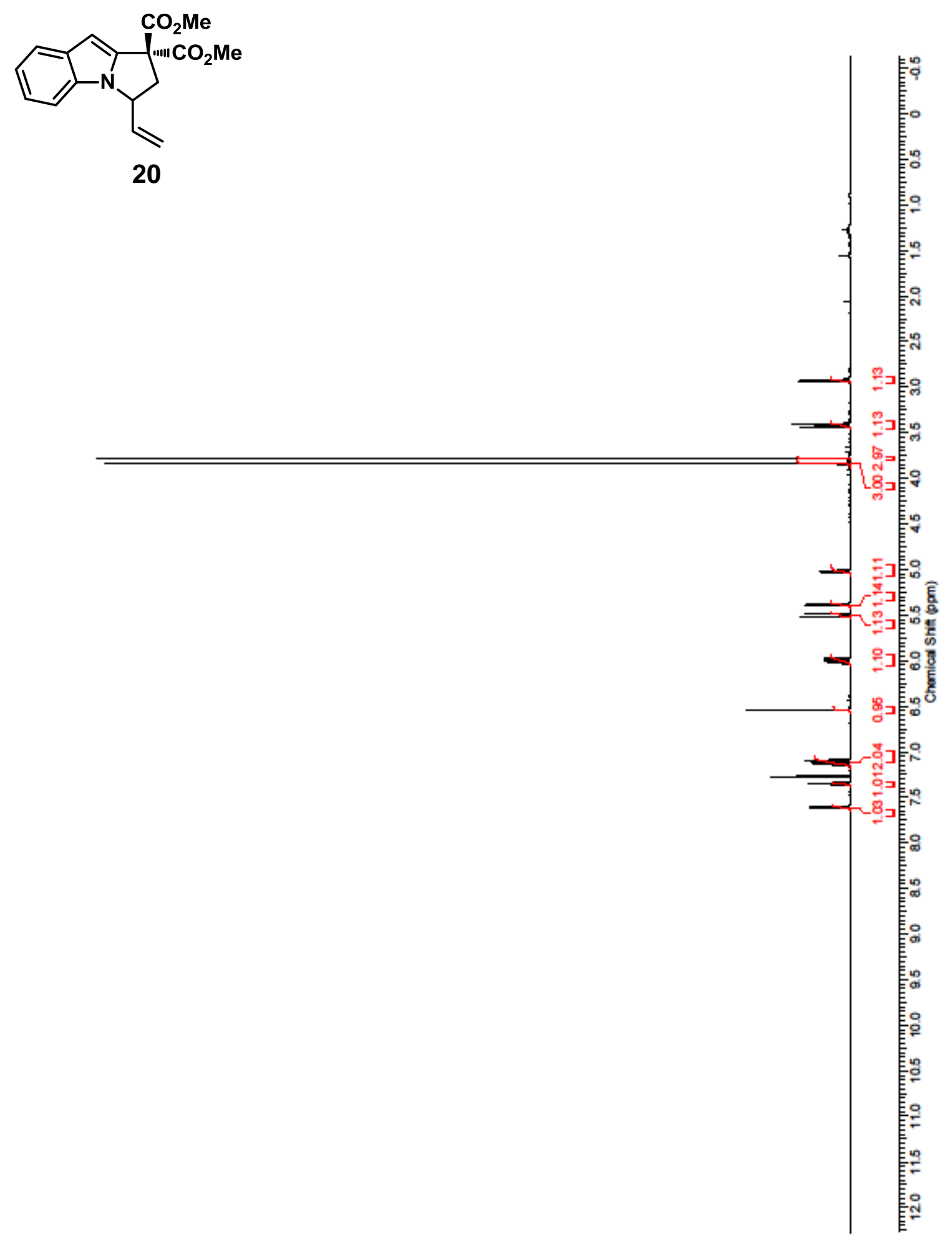

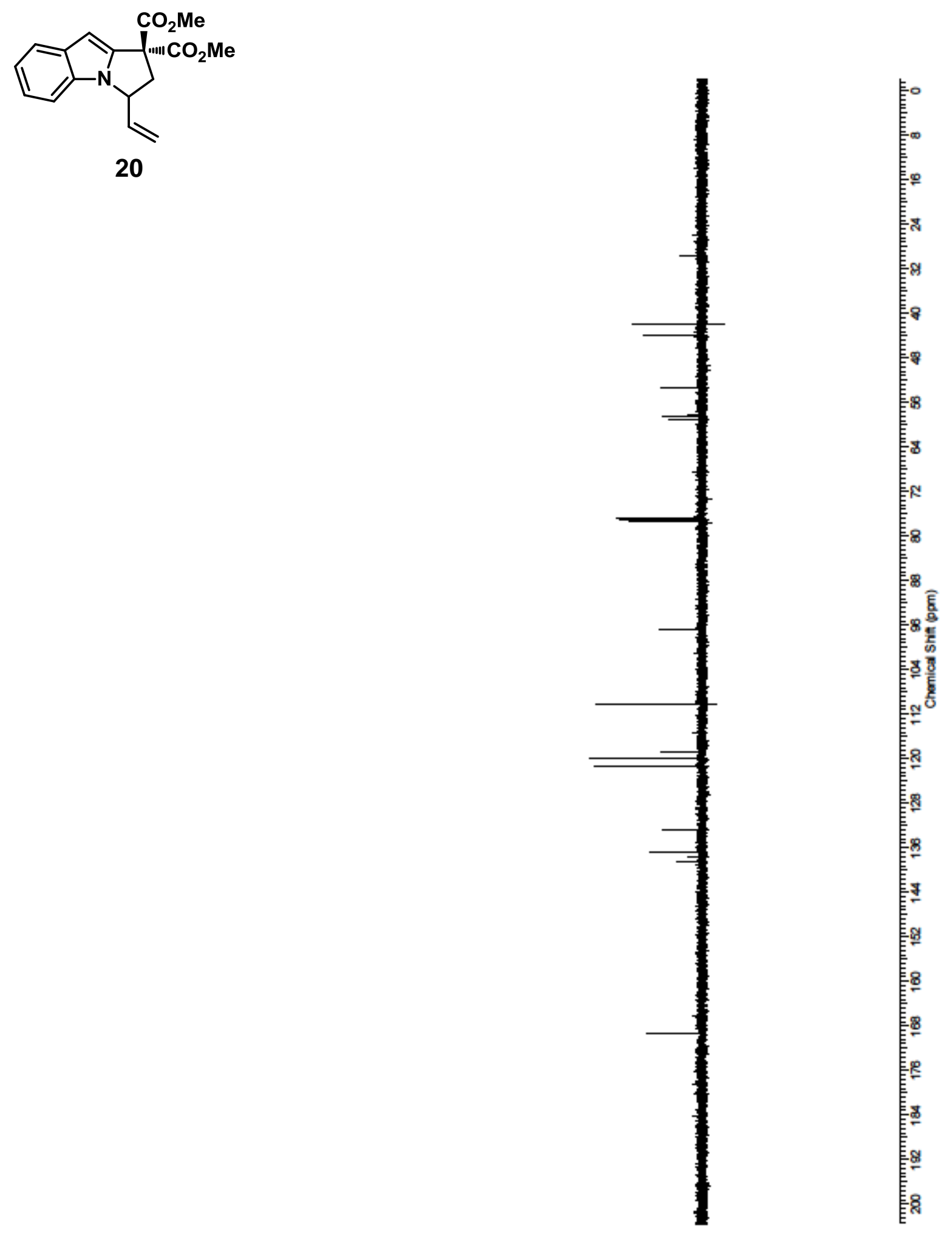

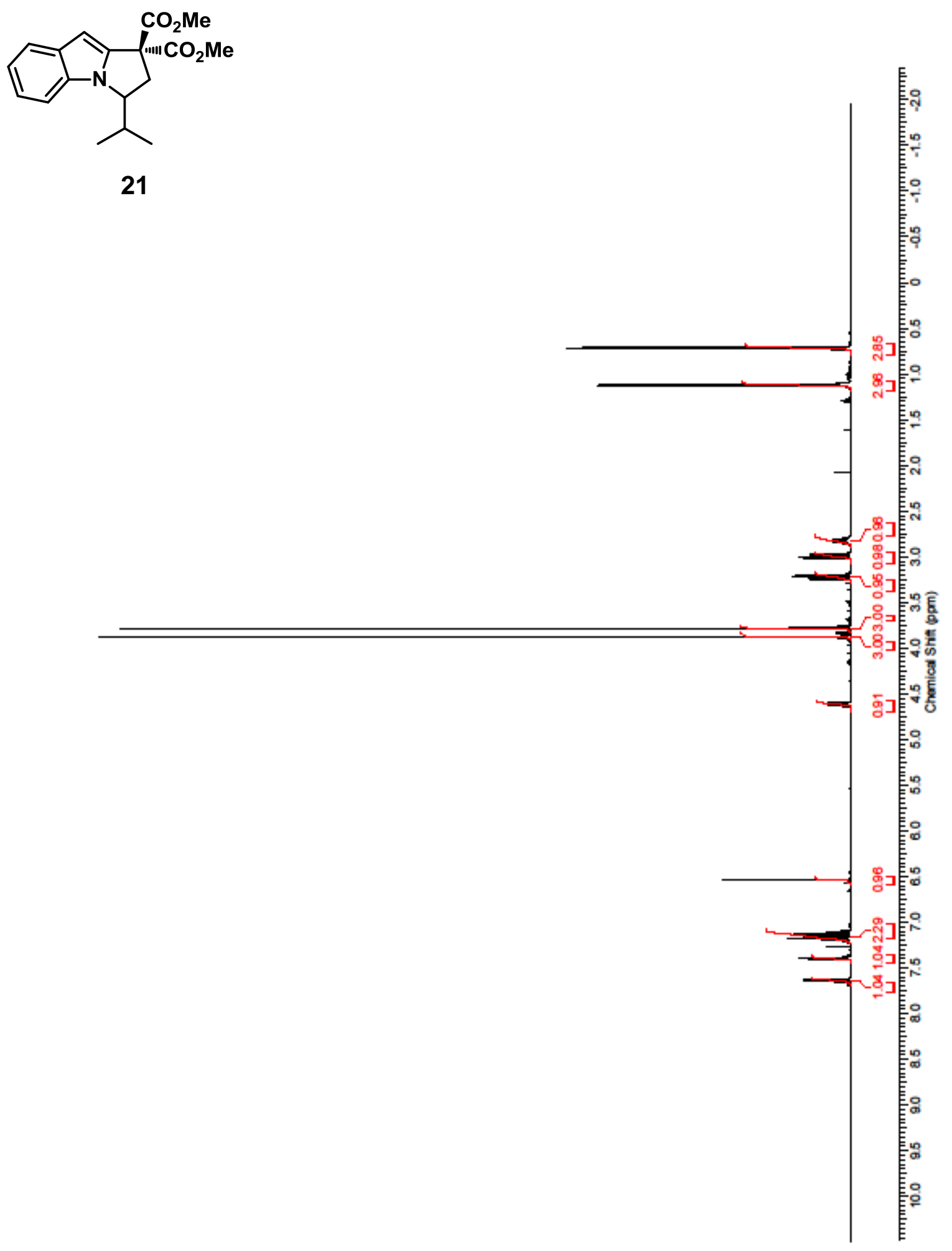


$$
1
$$



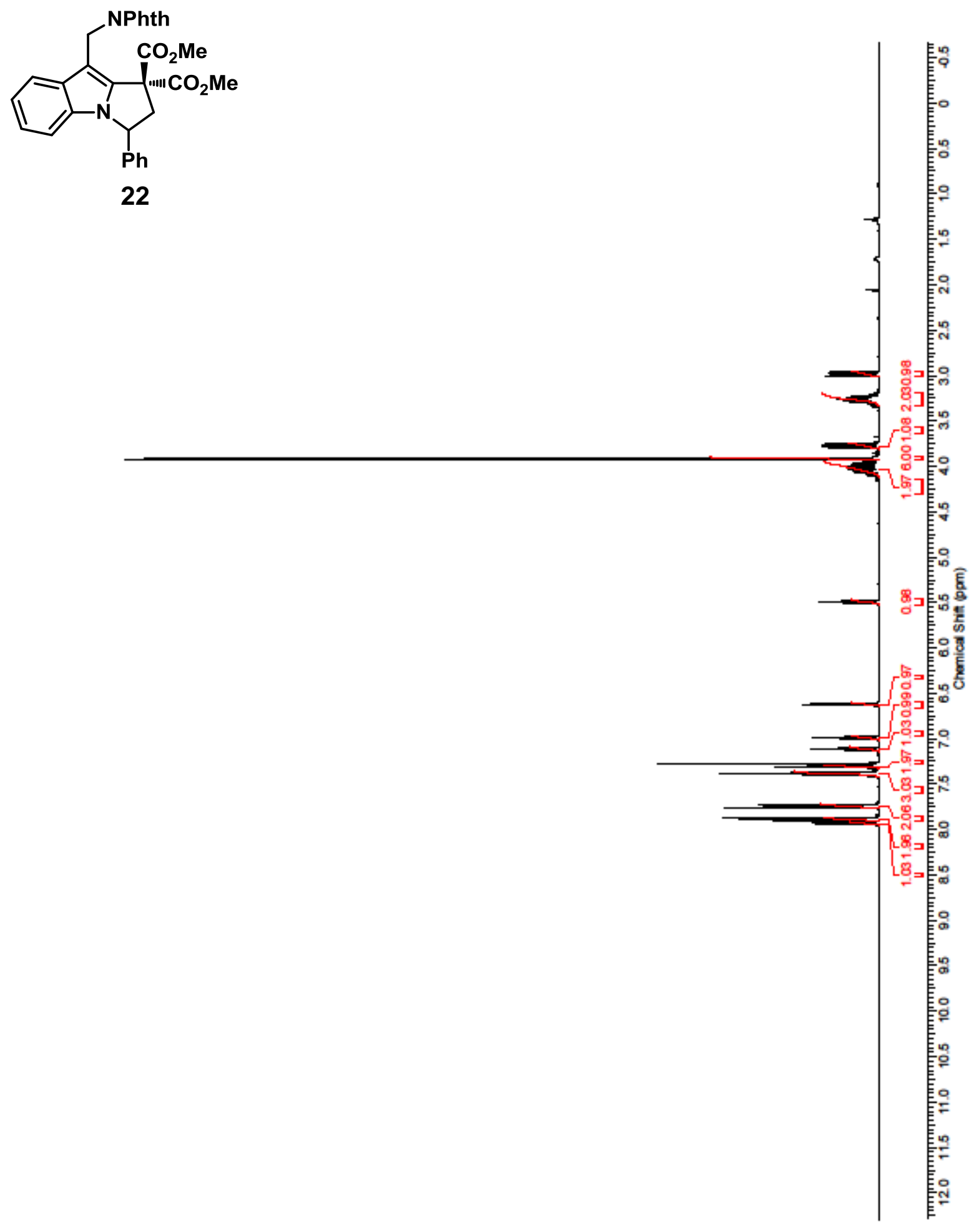

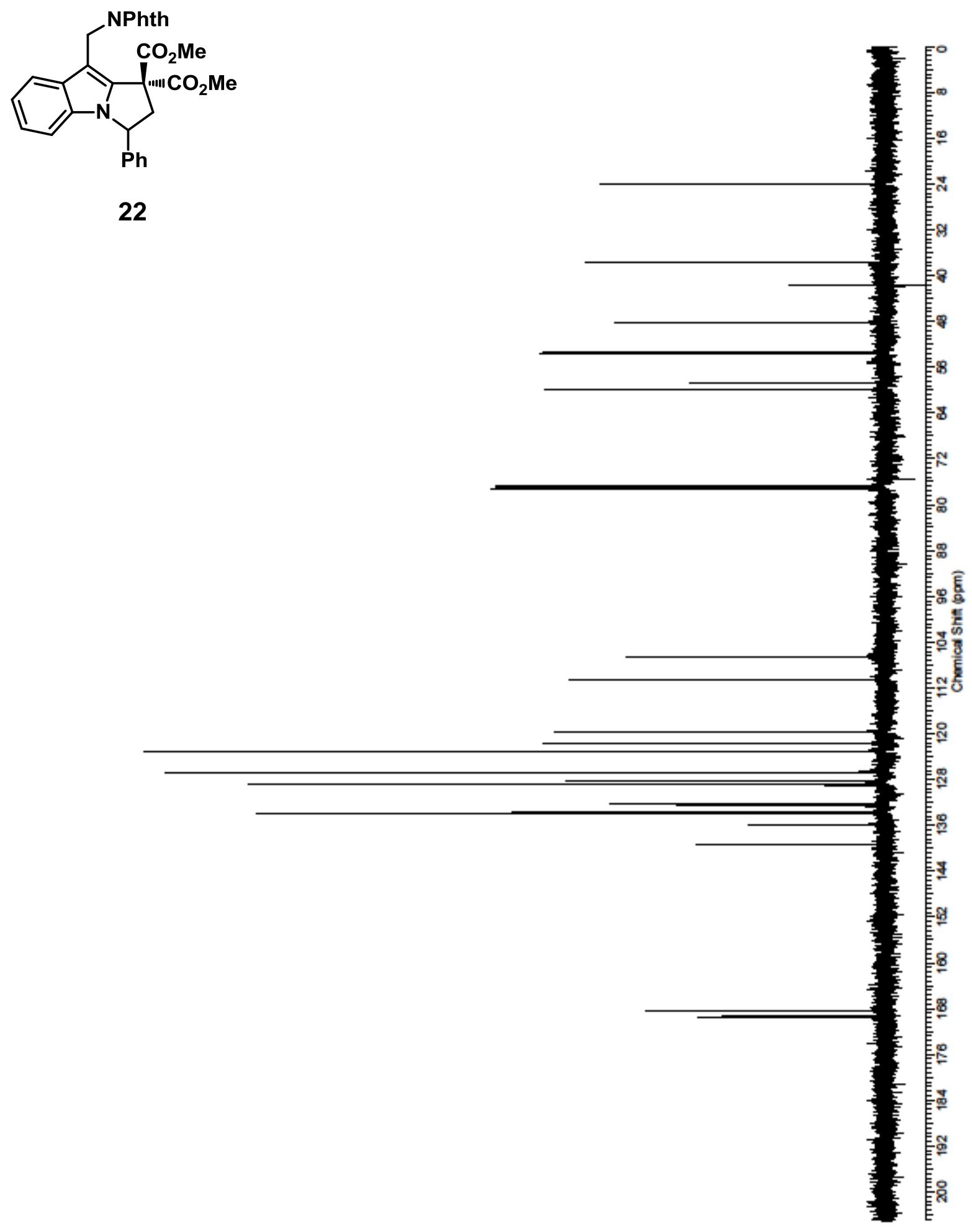


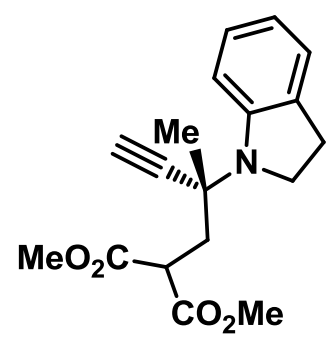

23

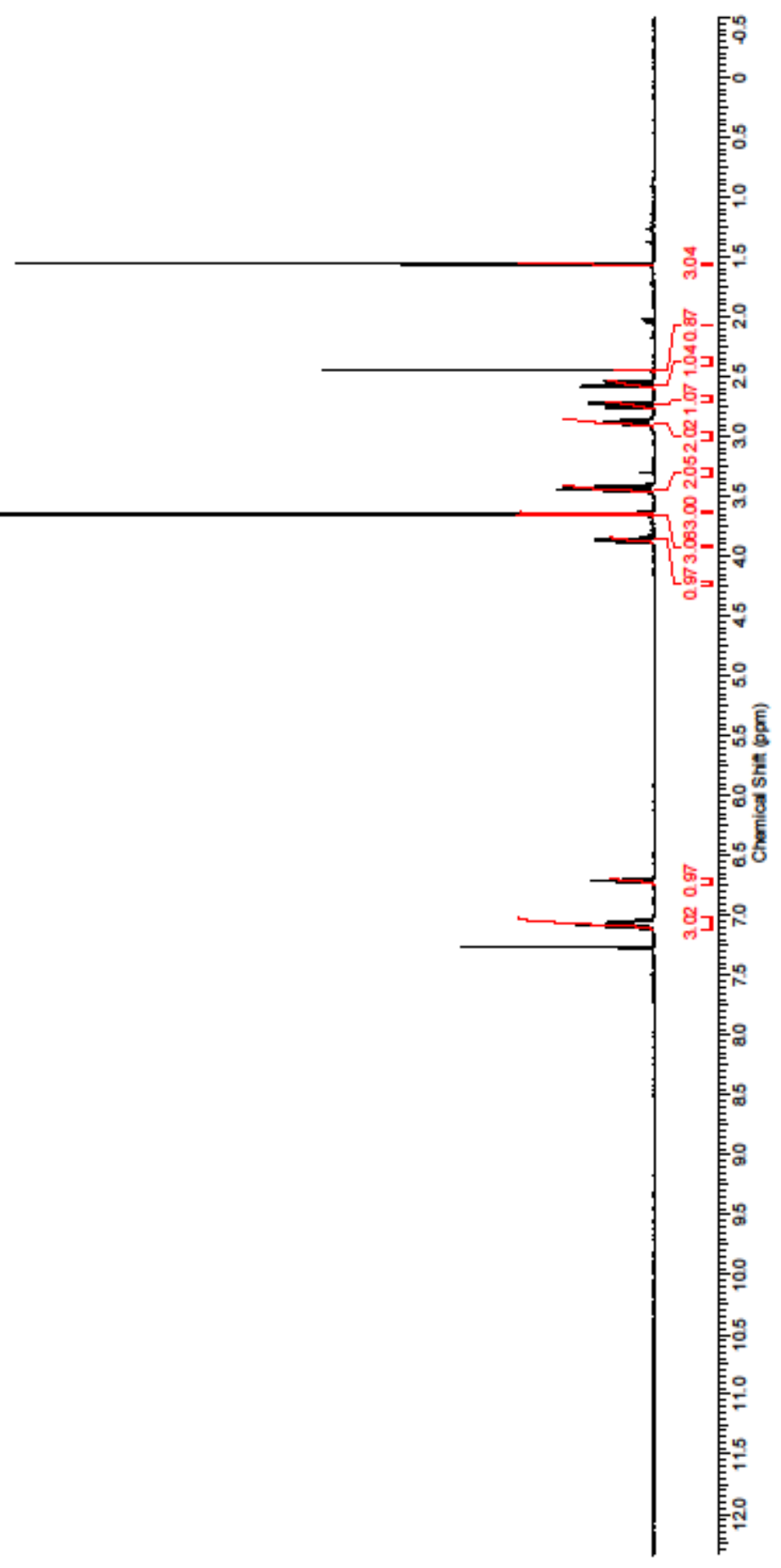




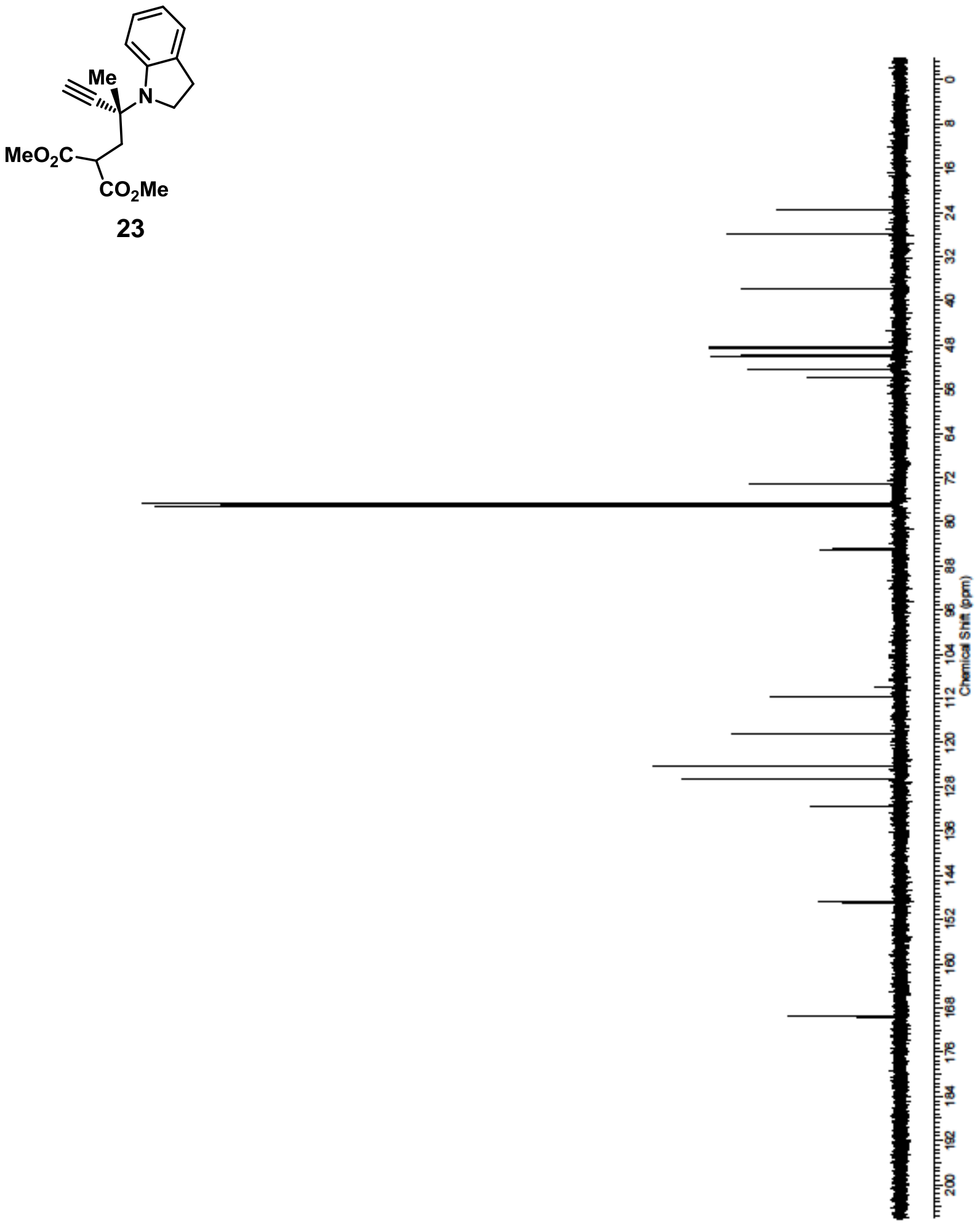

65 

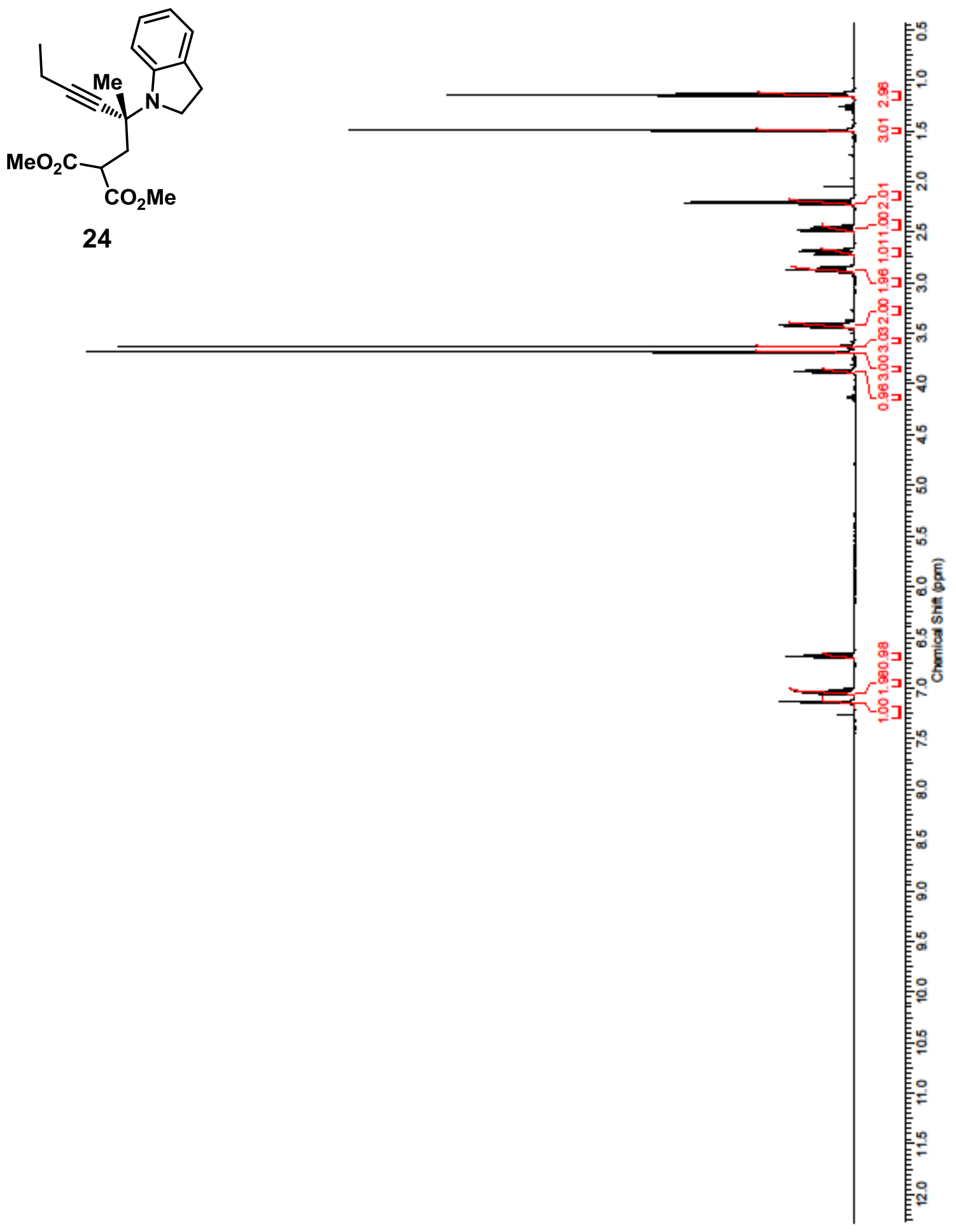


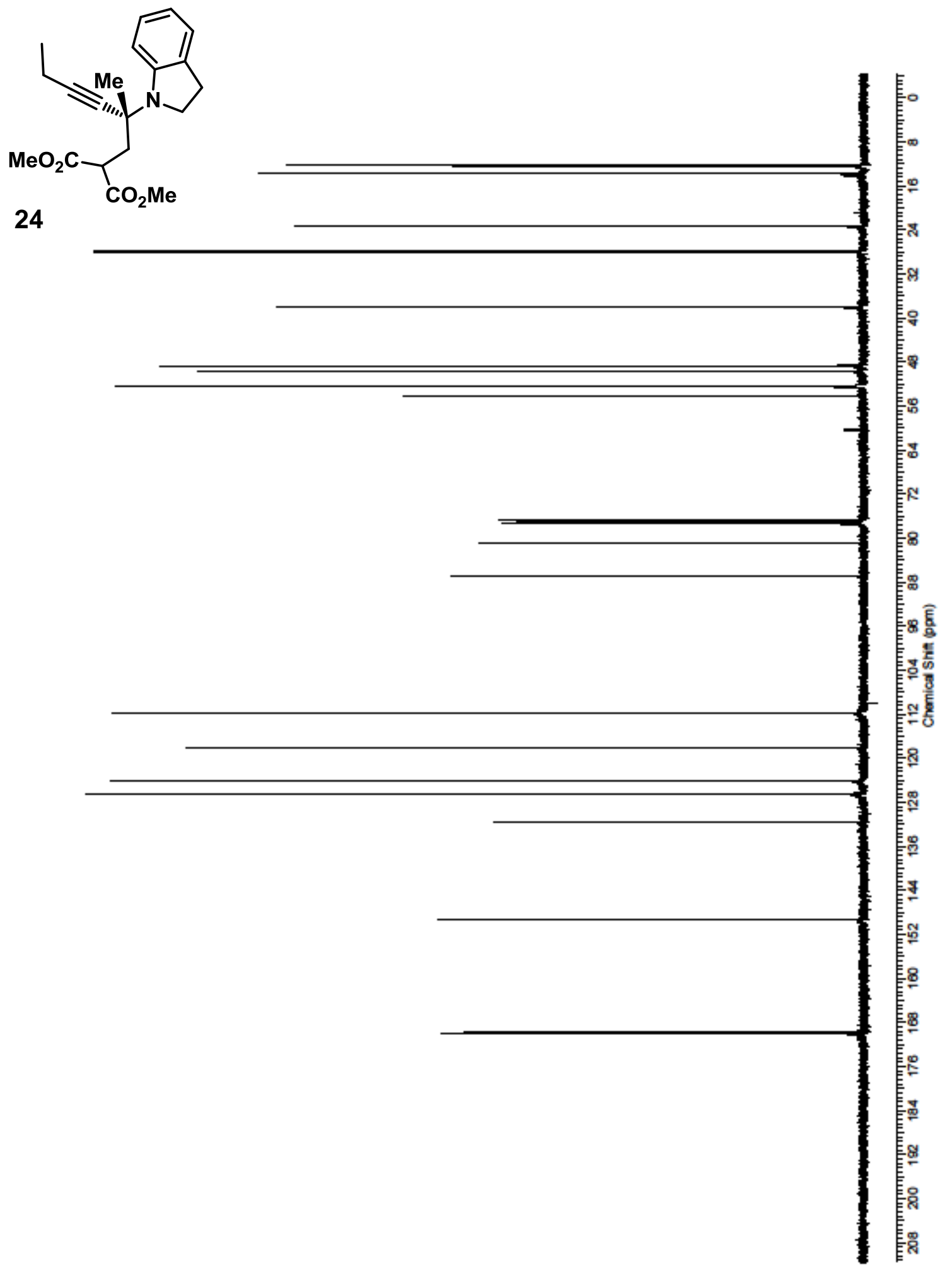




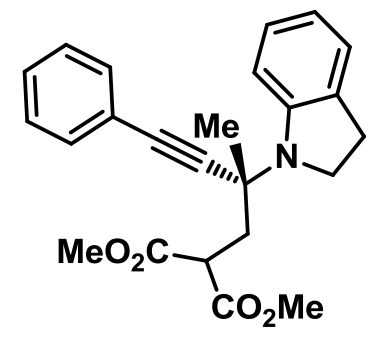

25

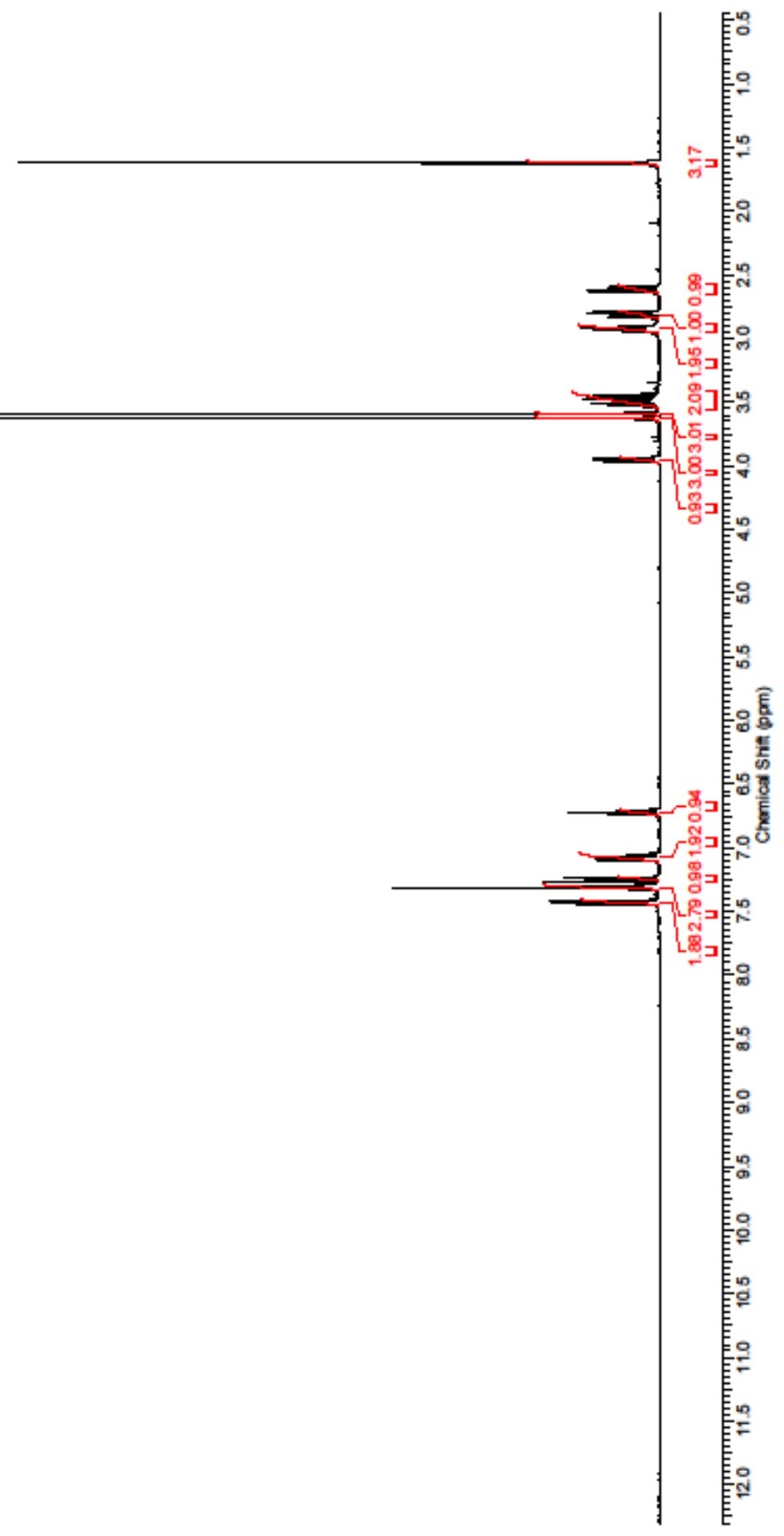




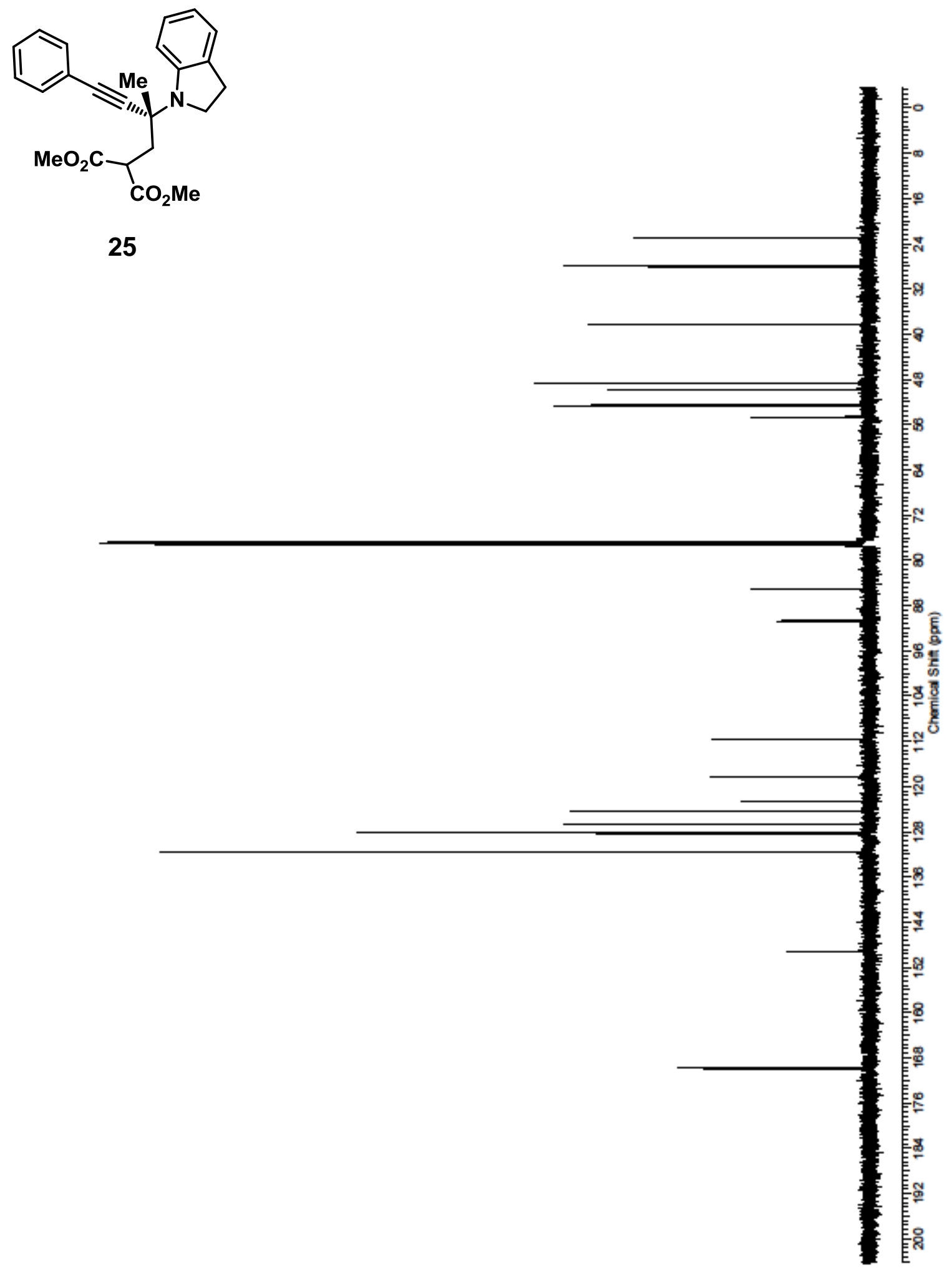



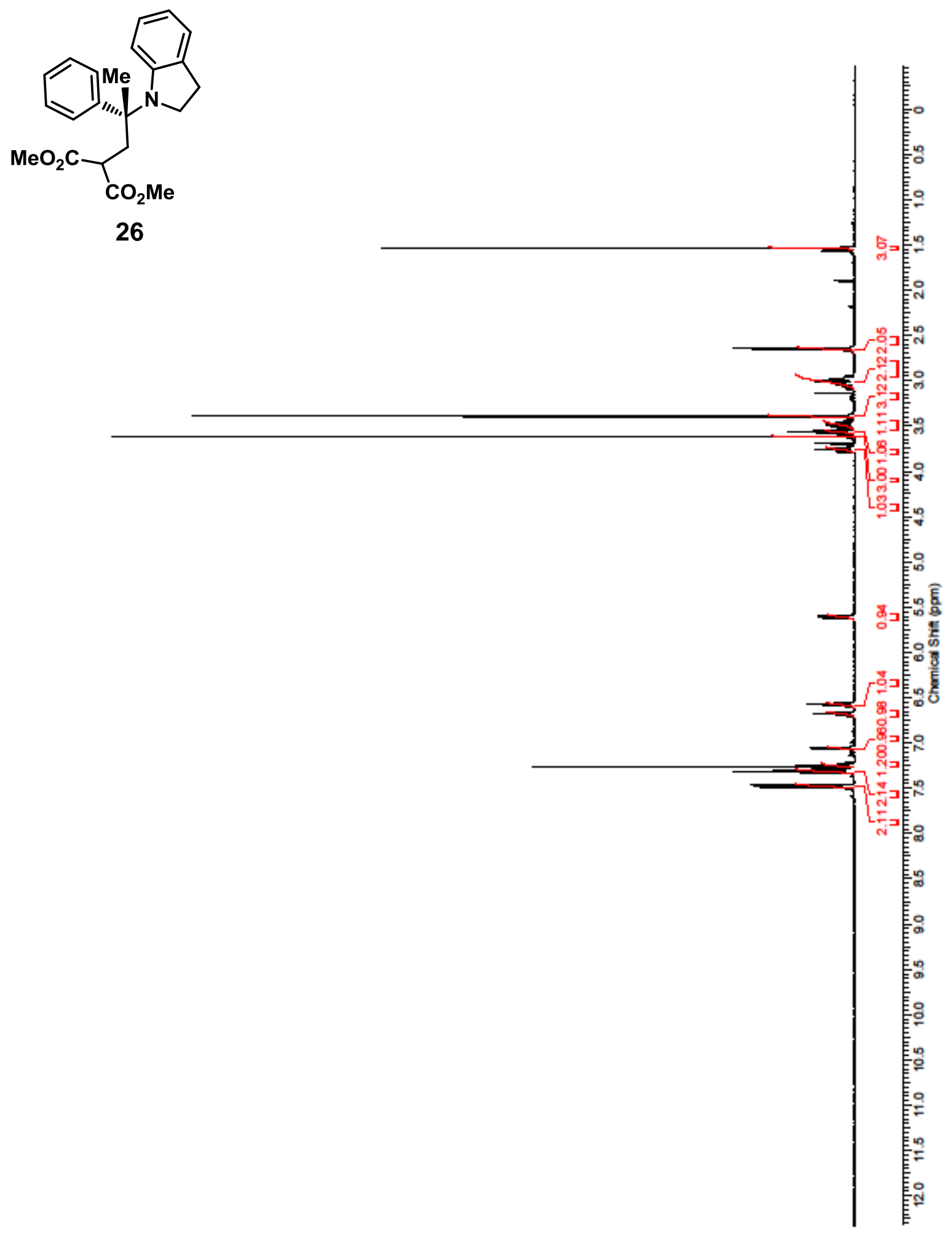

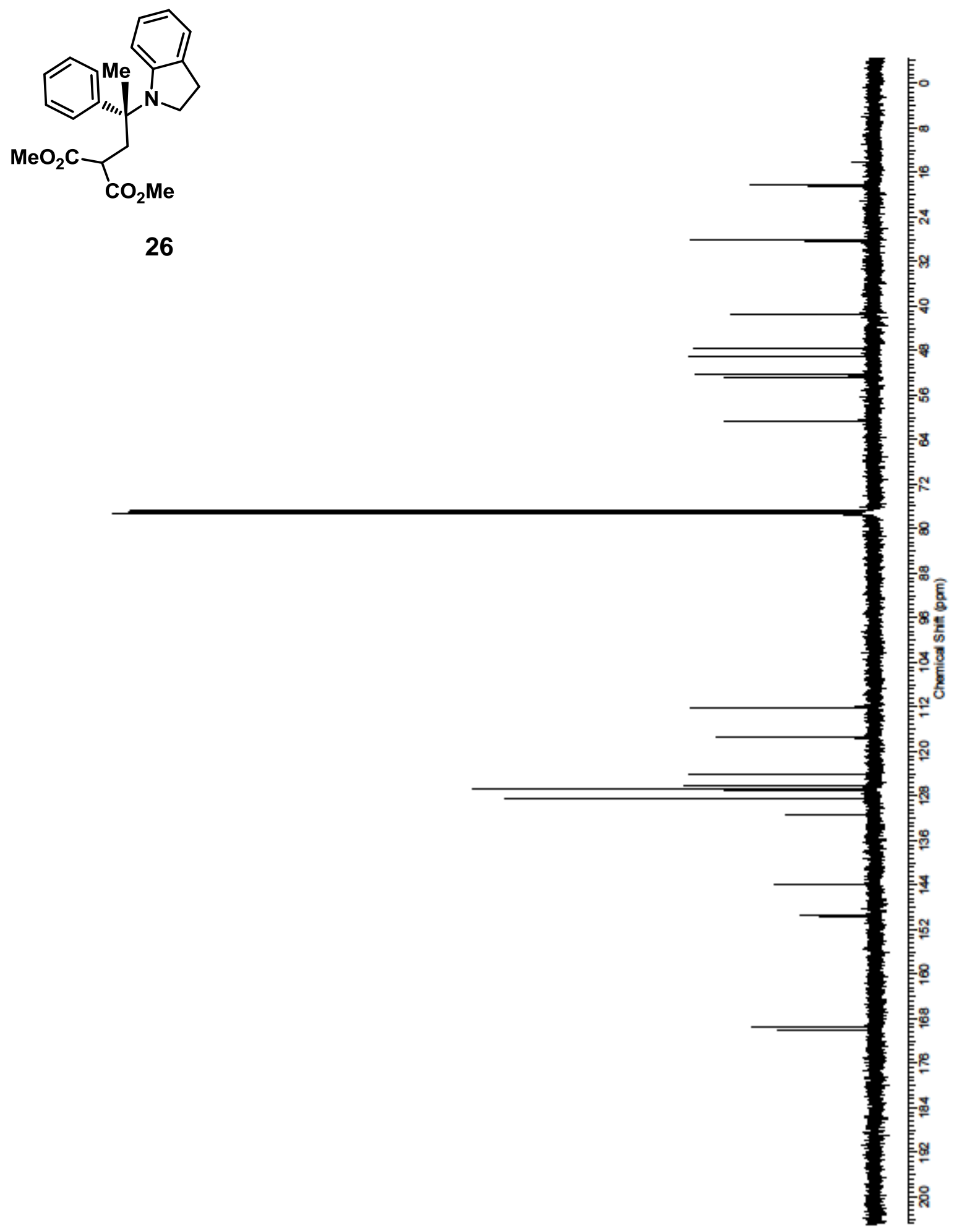

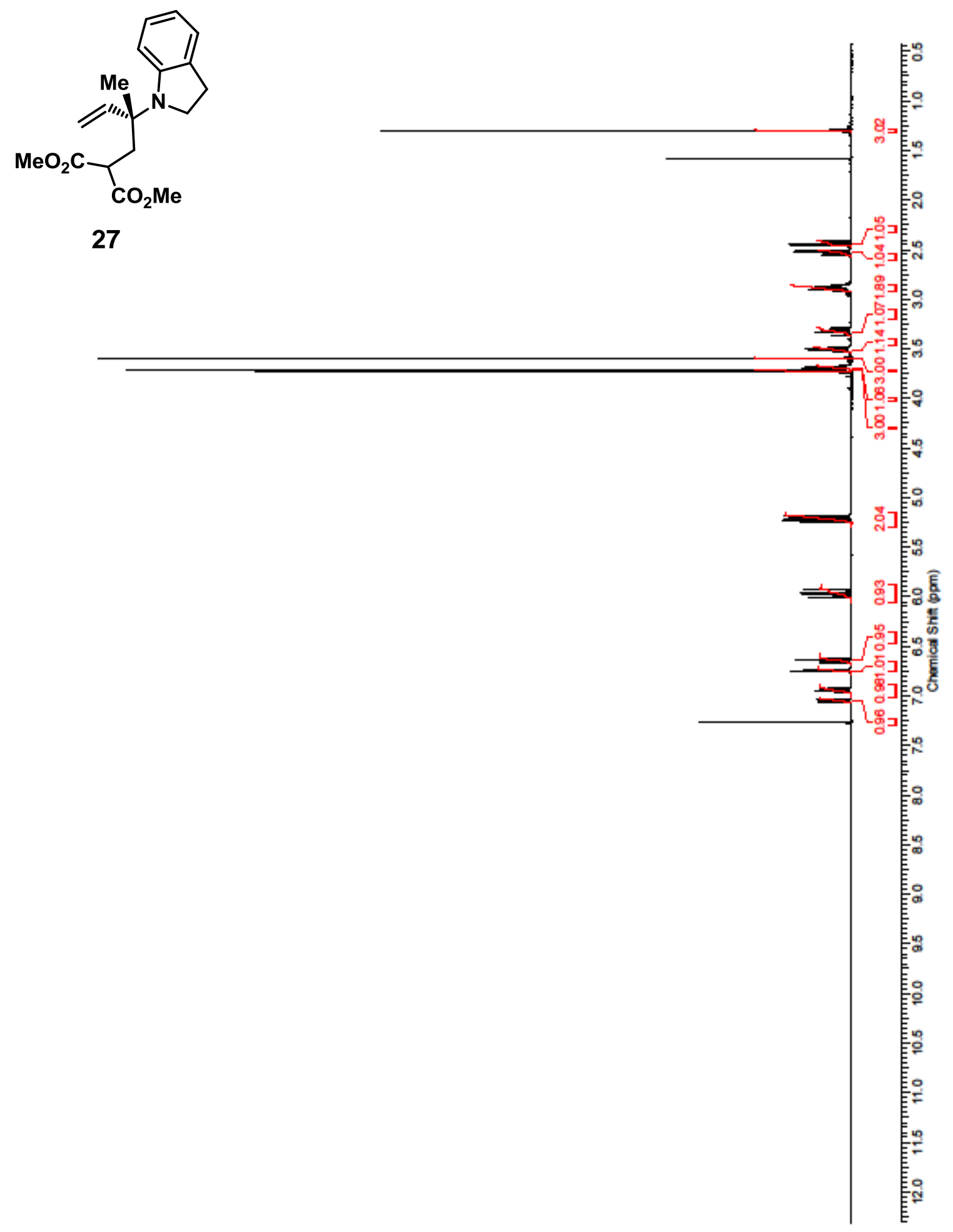

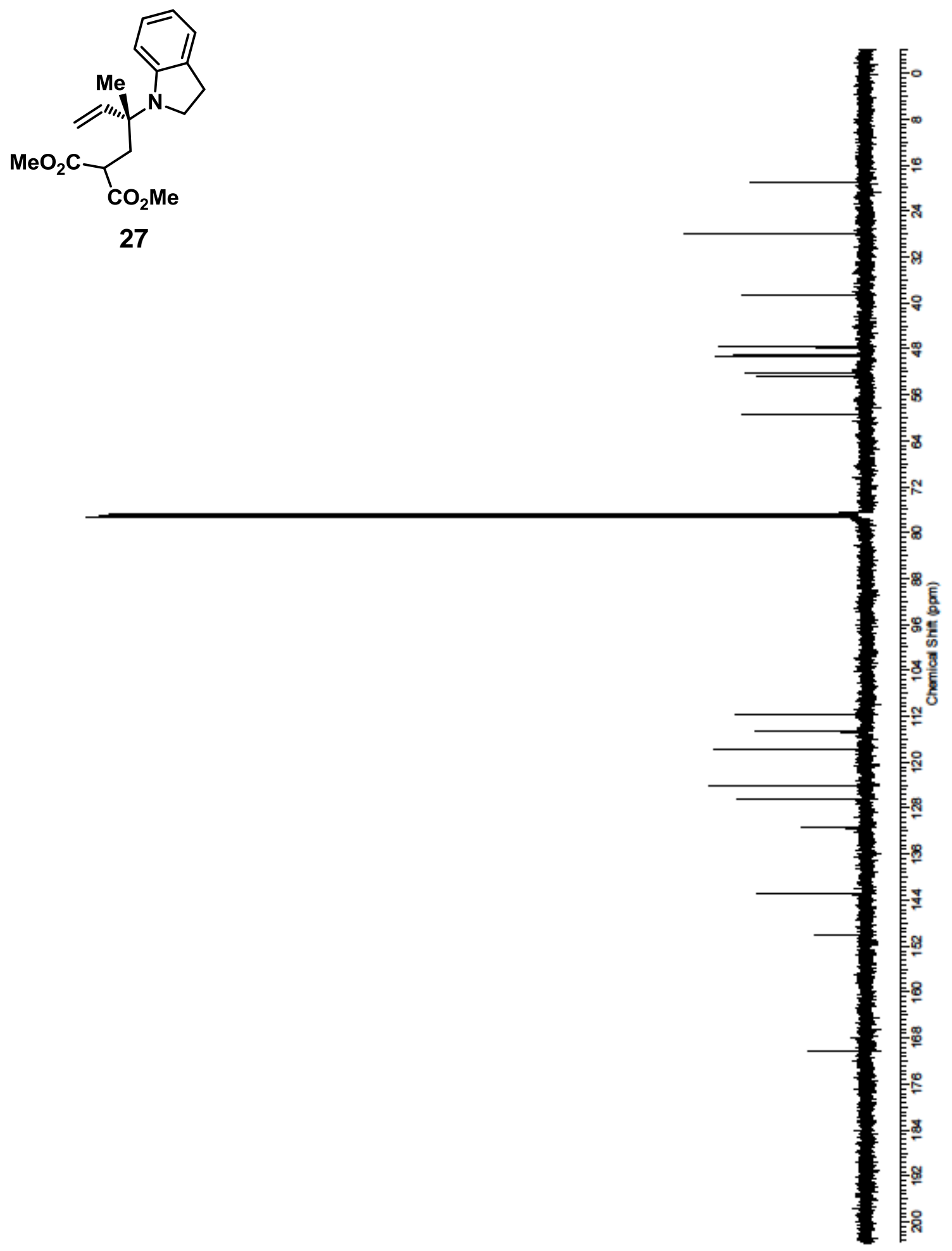

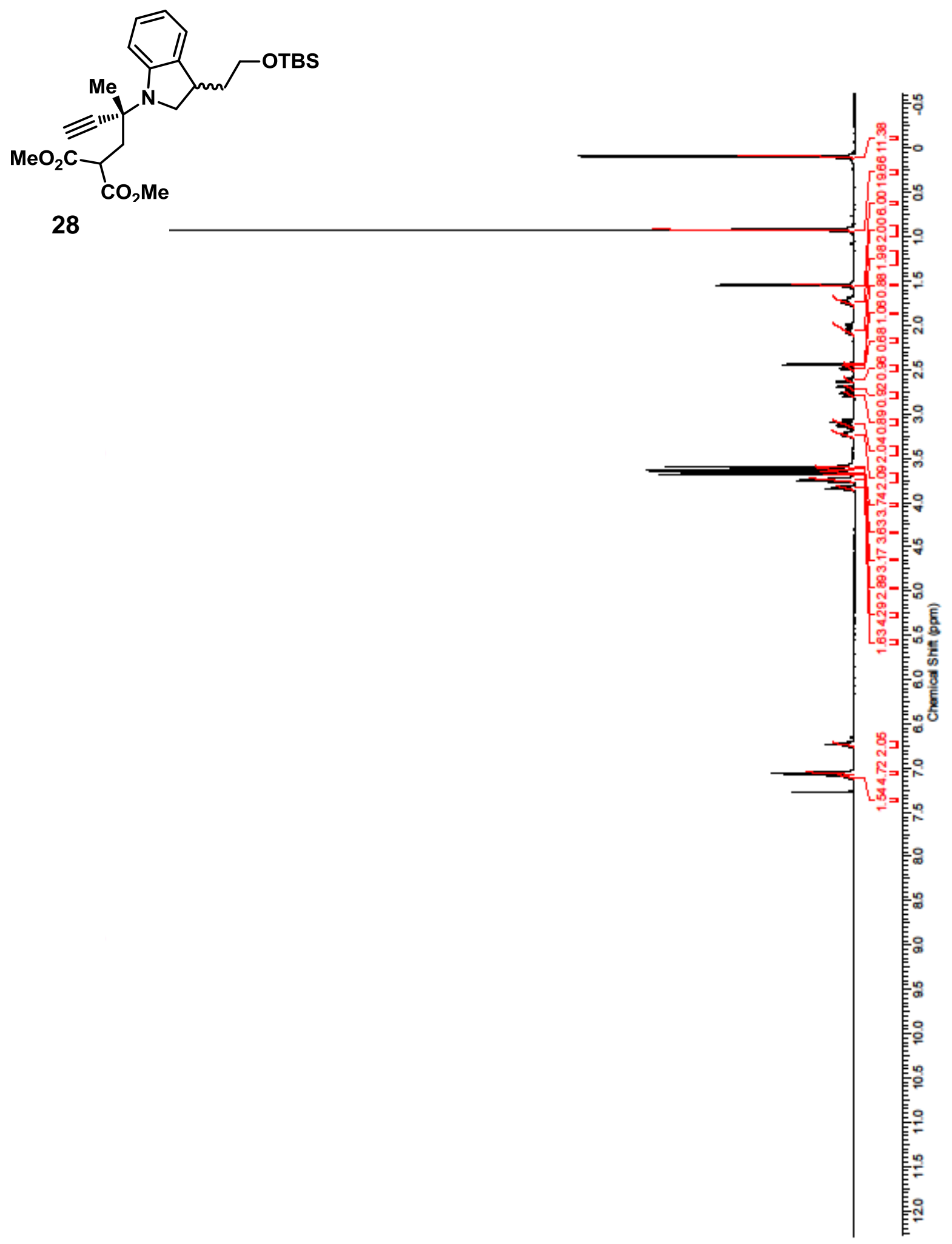


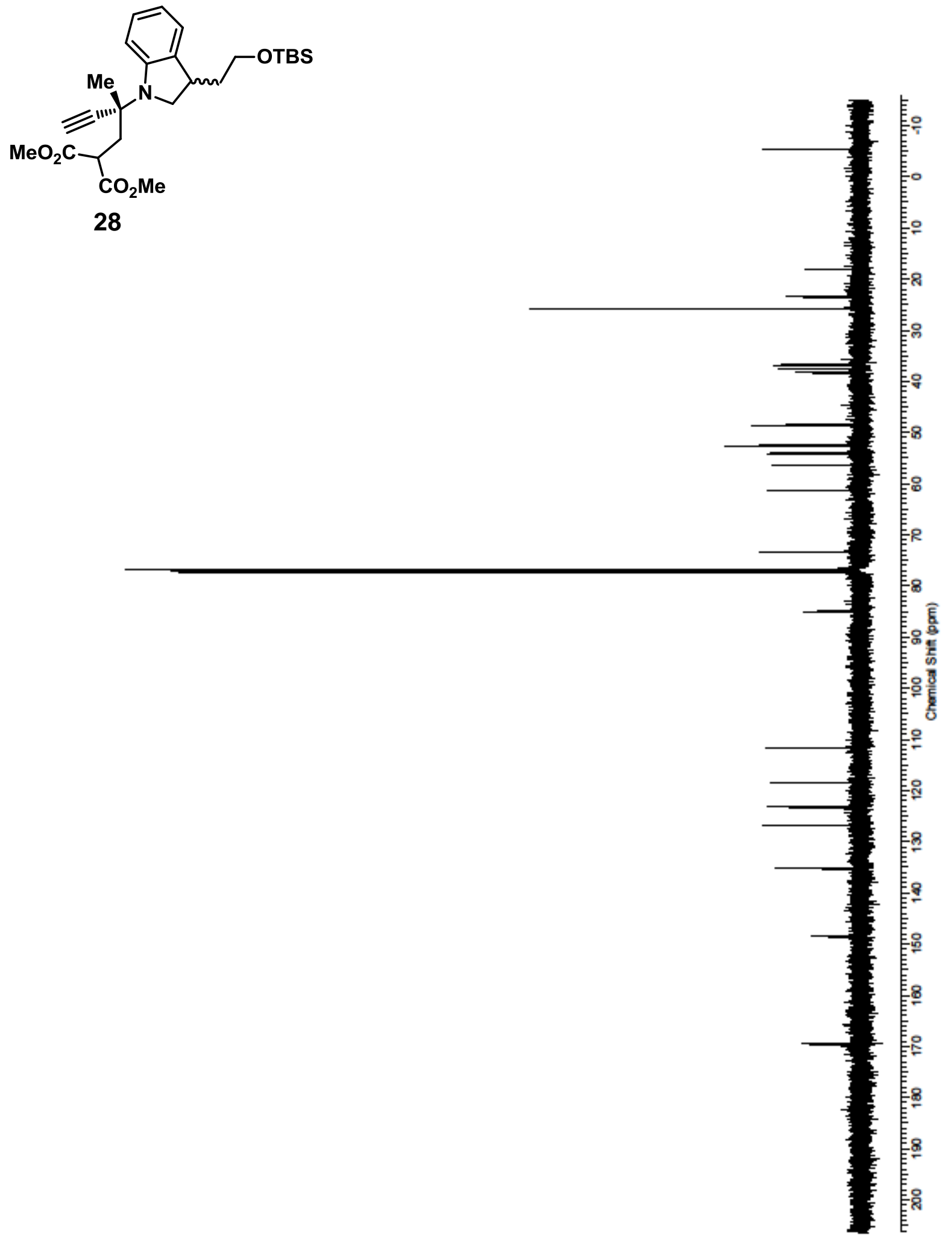

75 


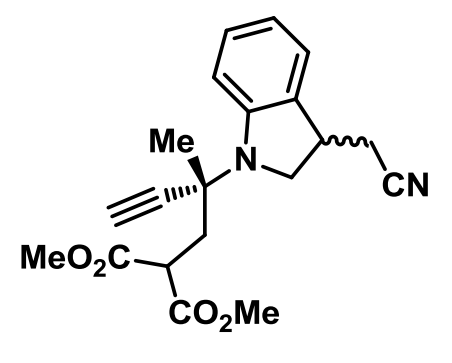

29

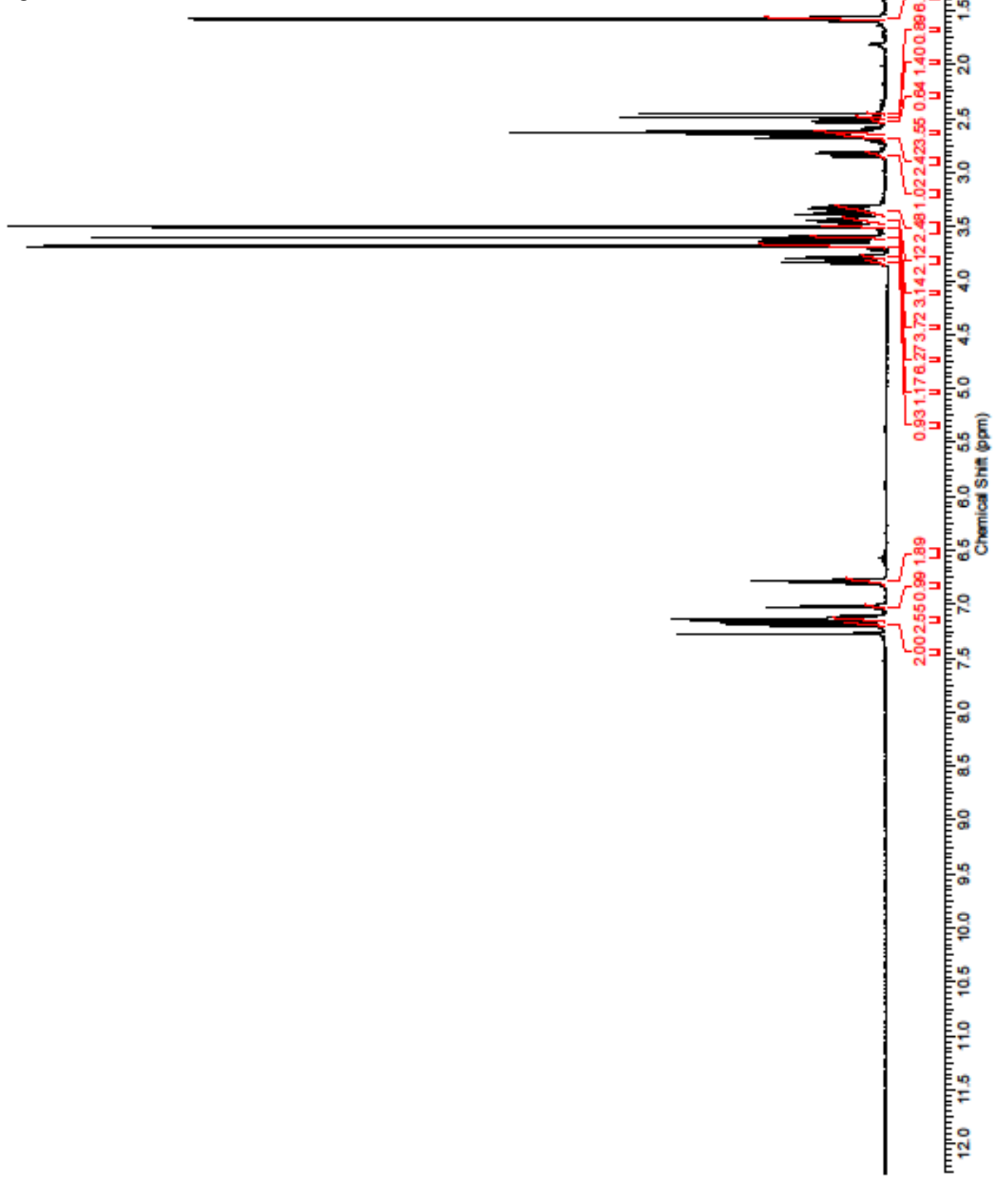




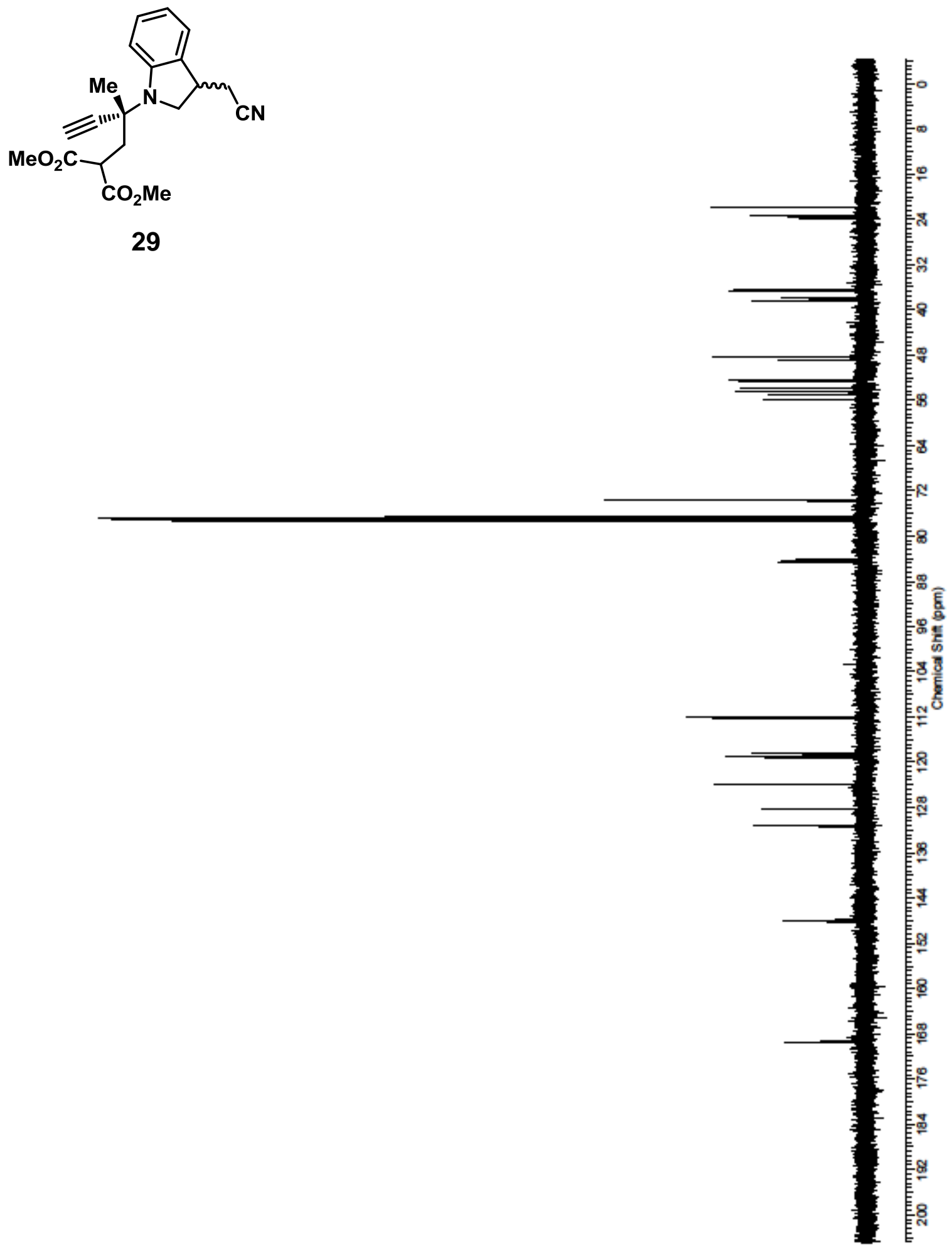

77 


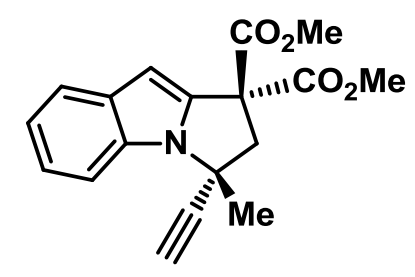

30

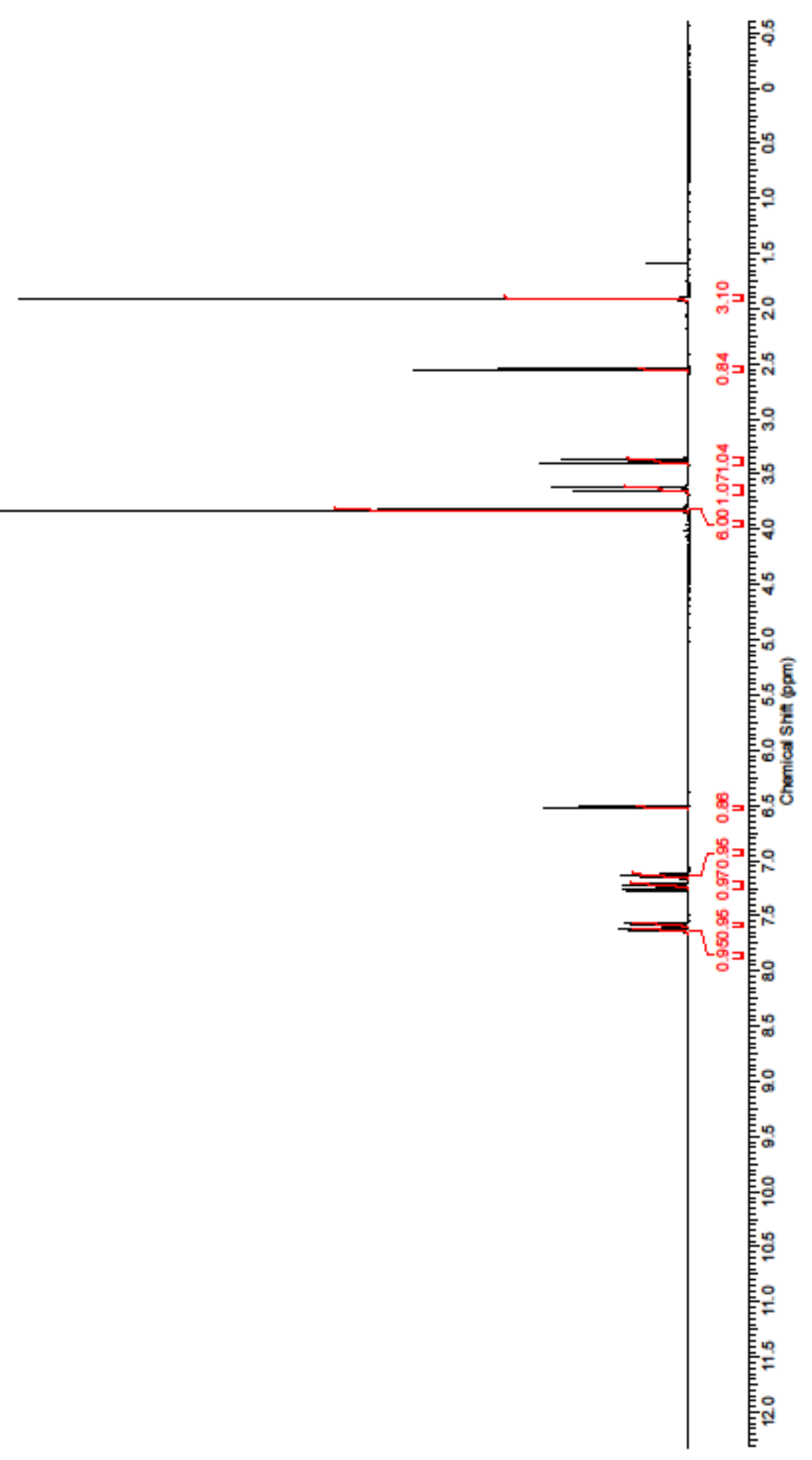



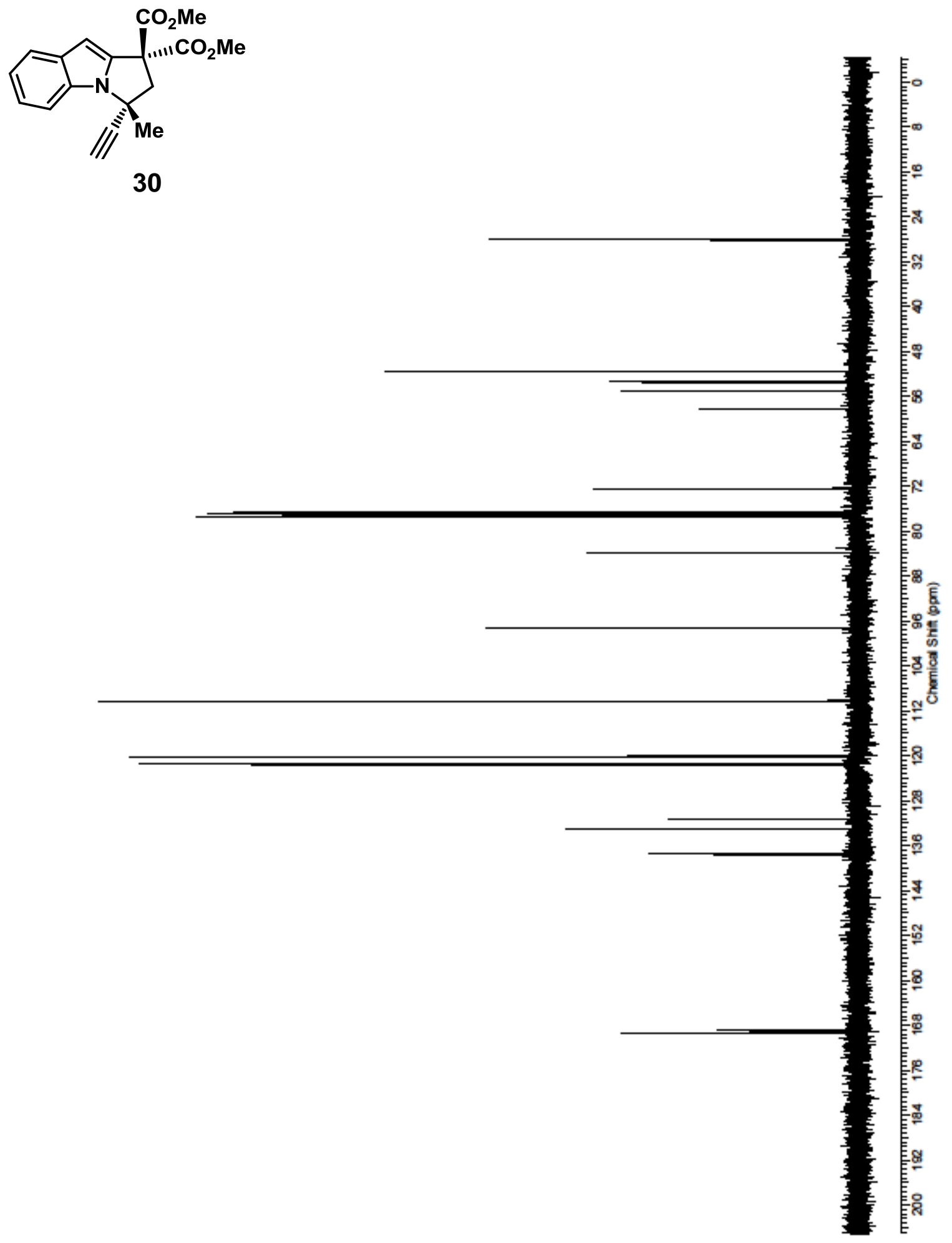


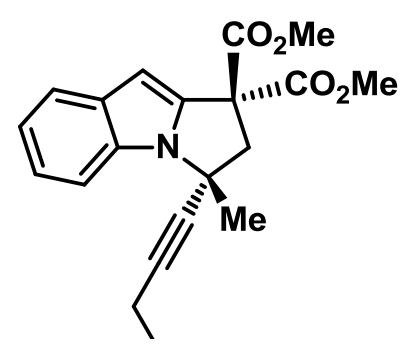

31

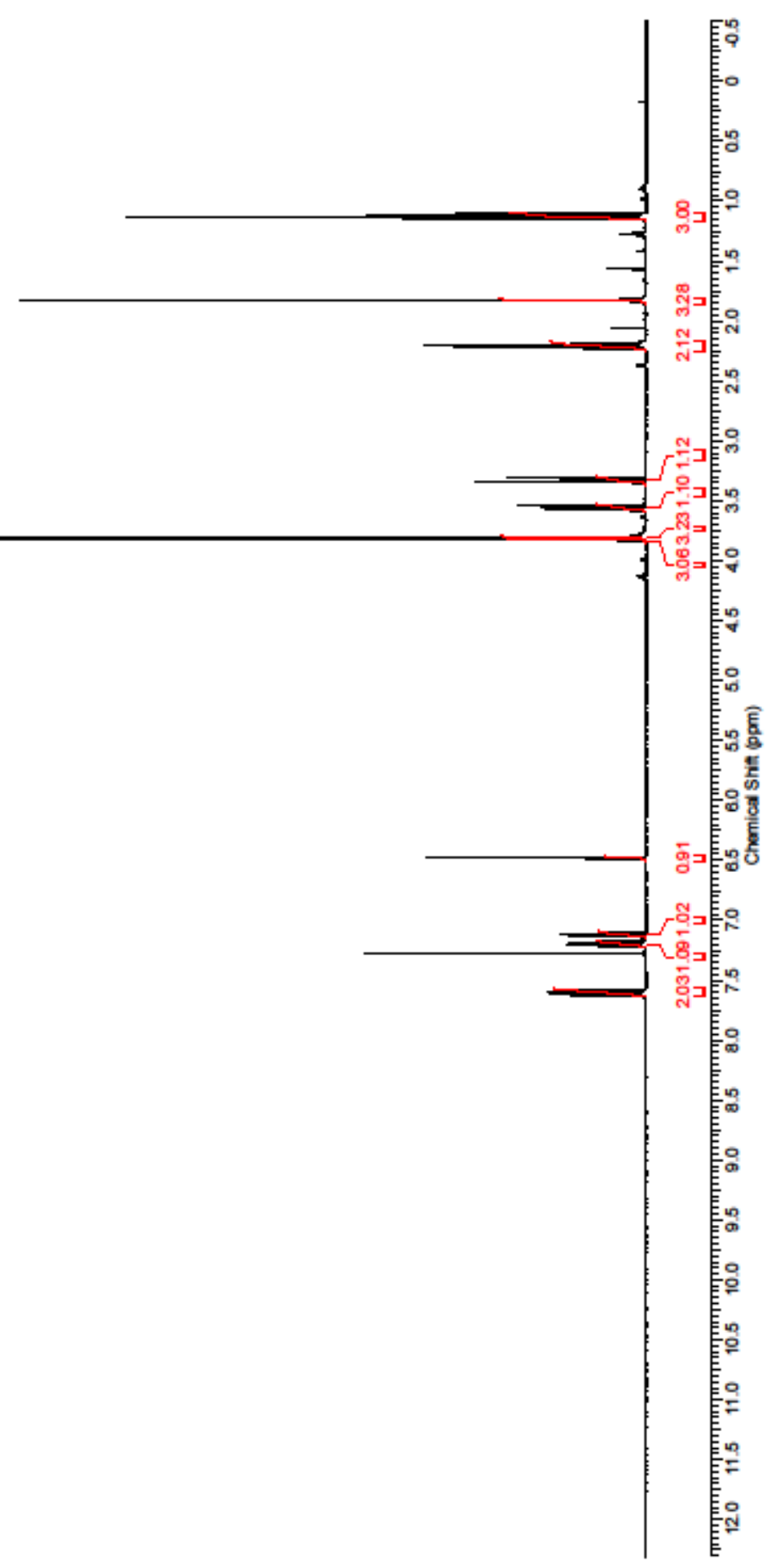



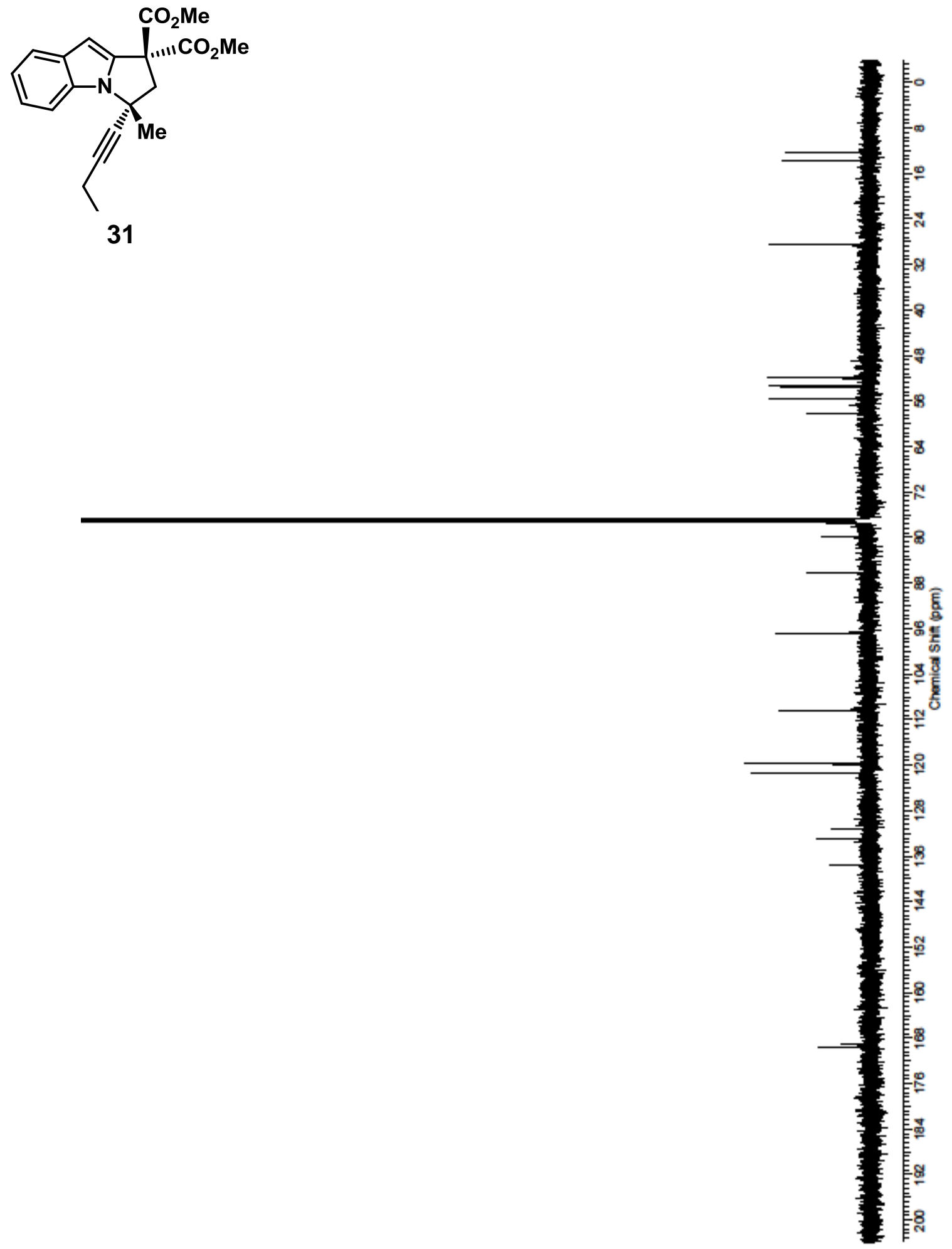

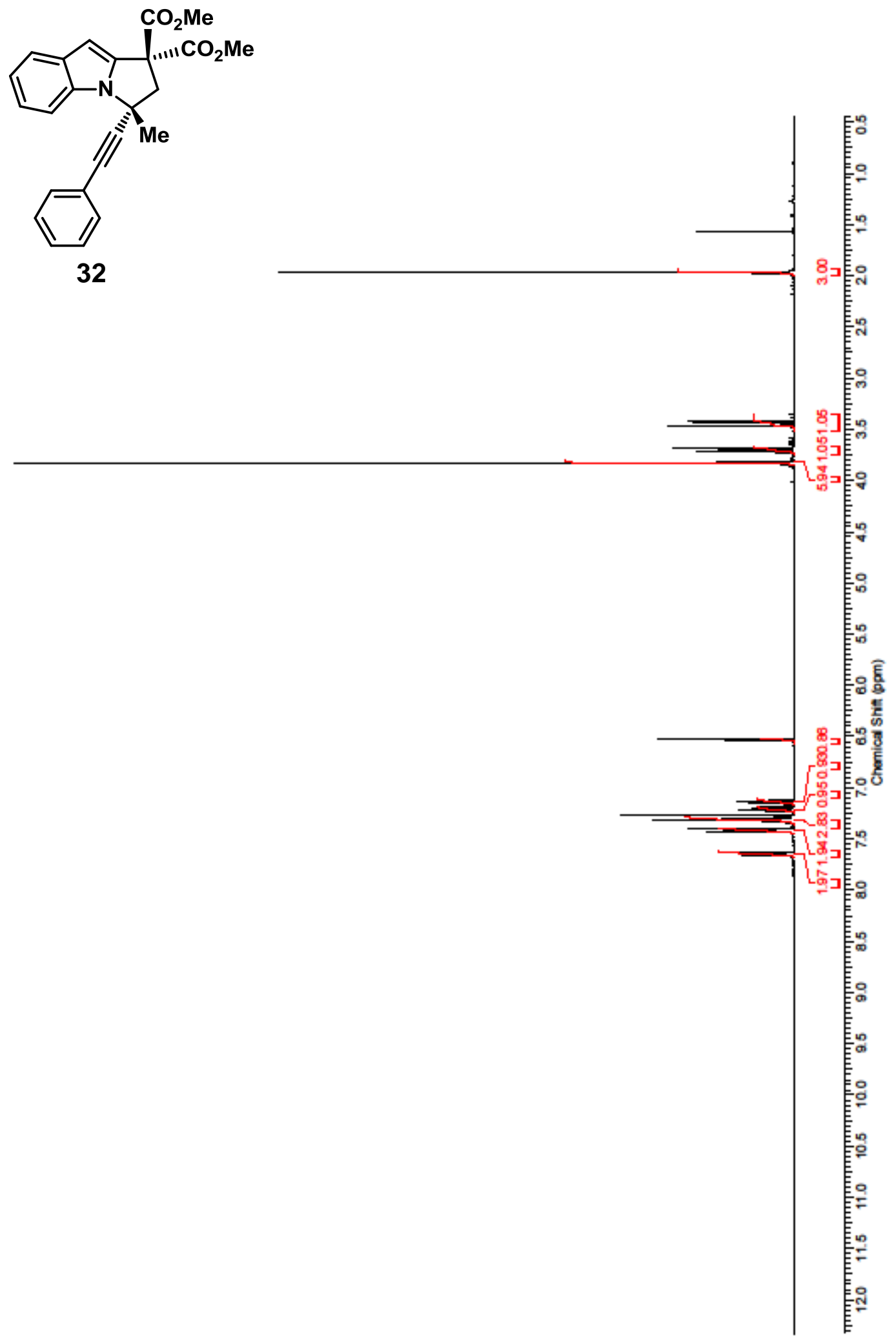


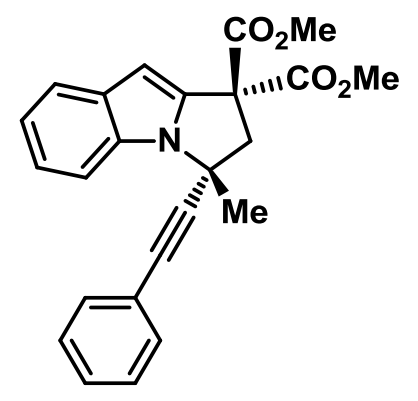

32

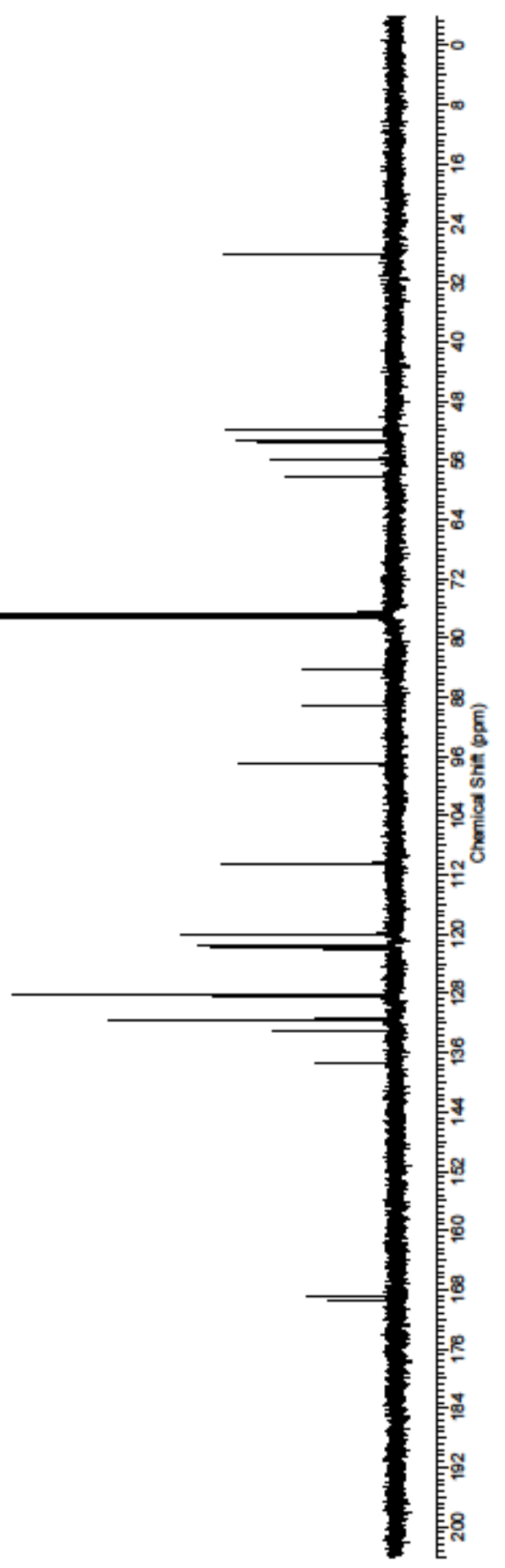




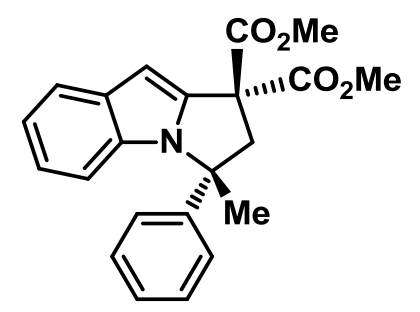

33

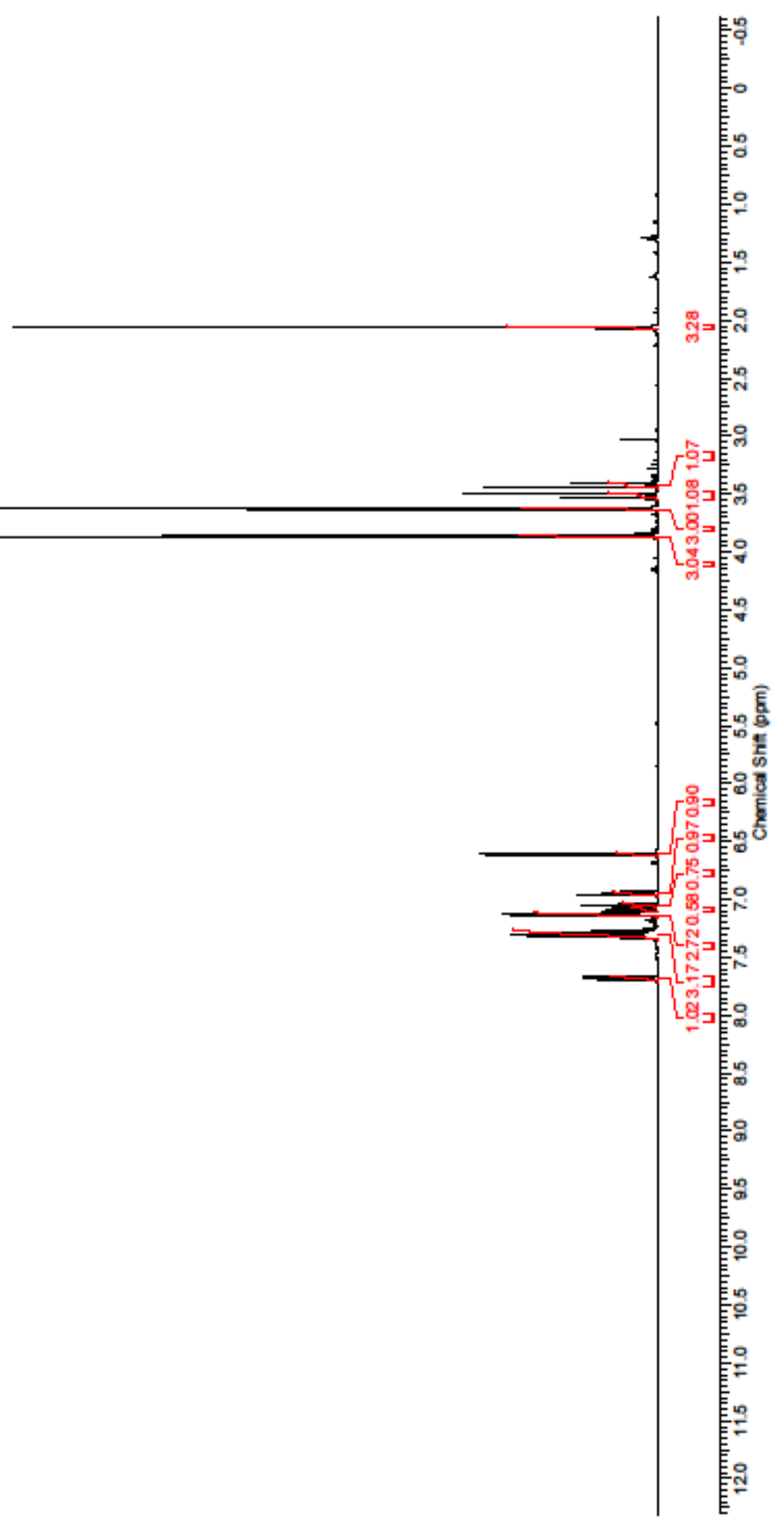




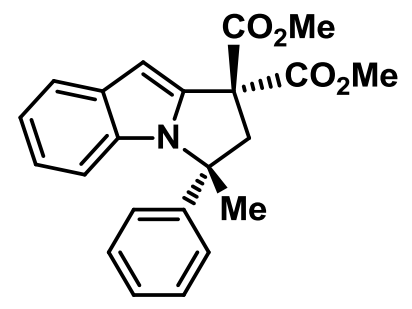

33

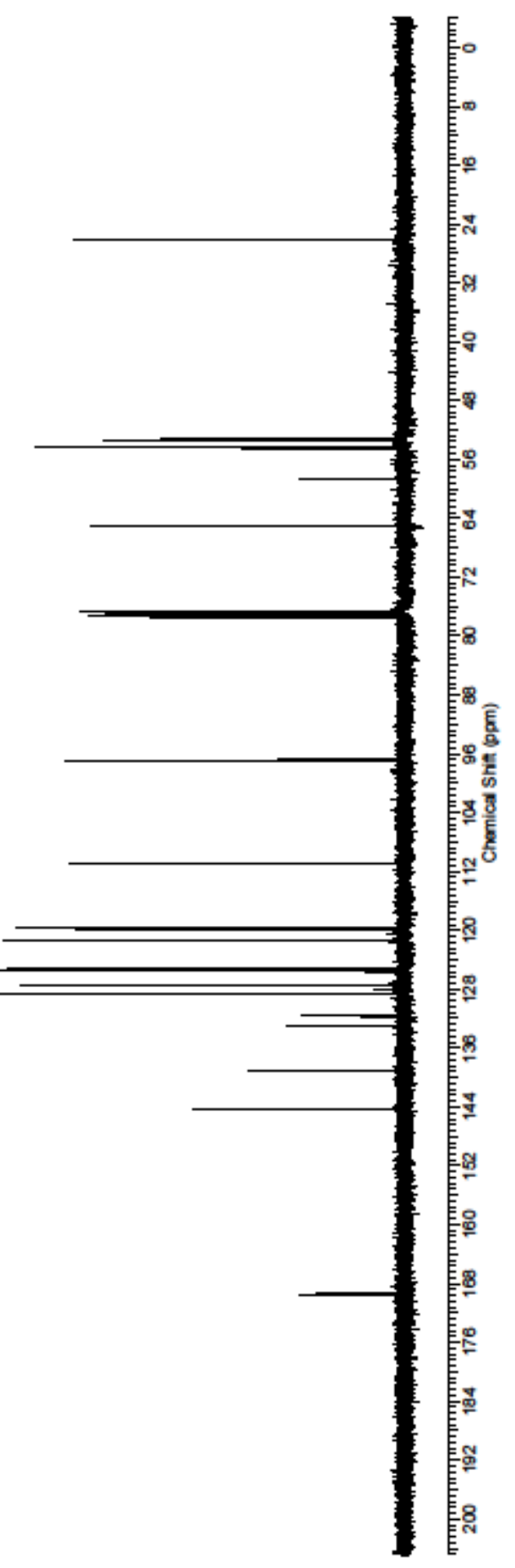




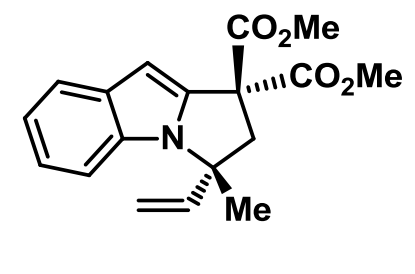

34

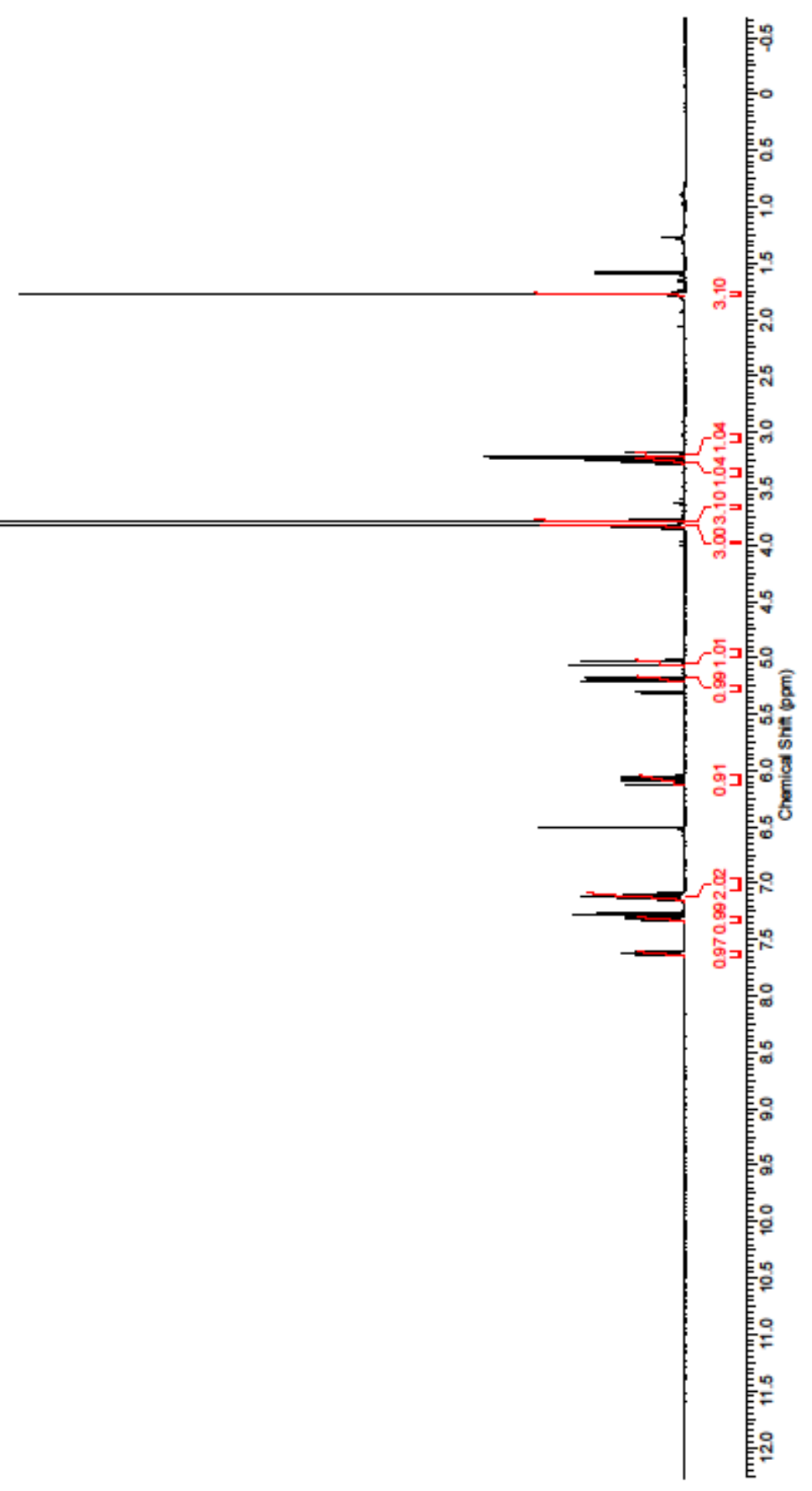




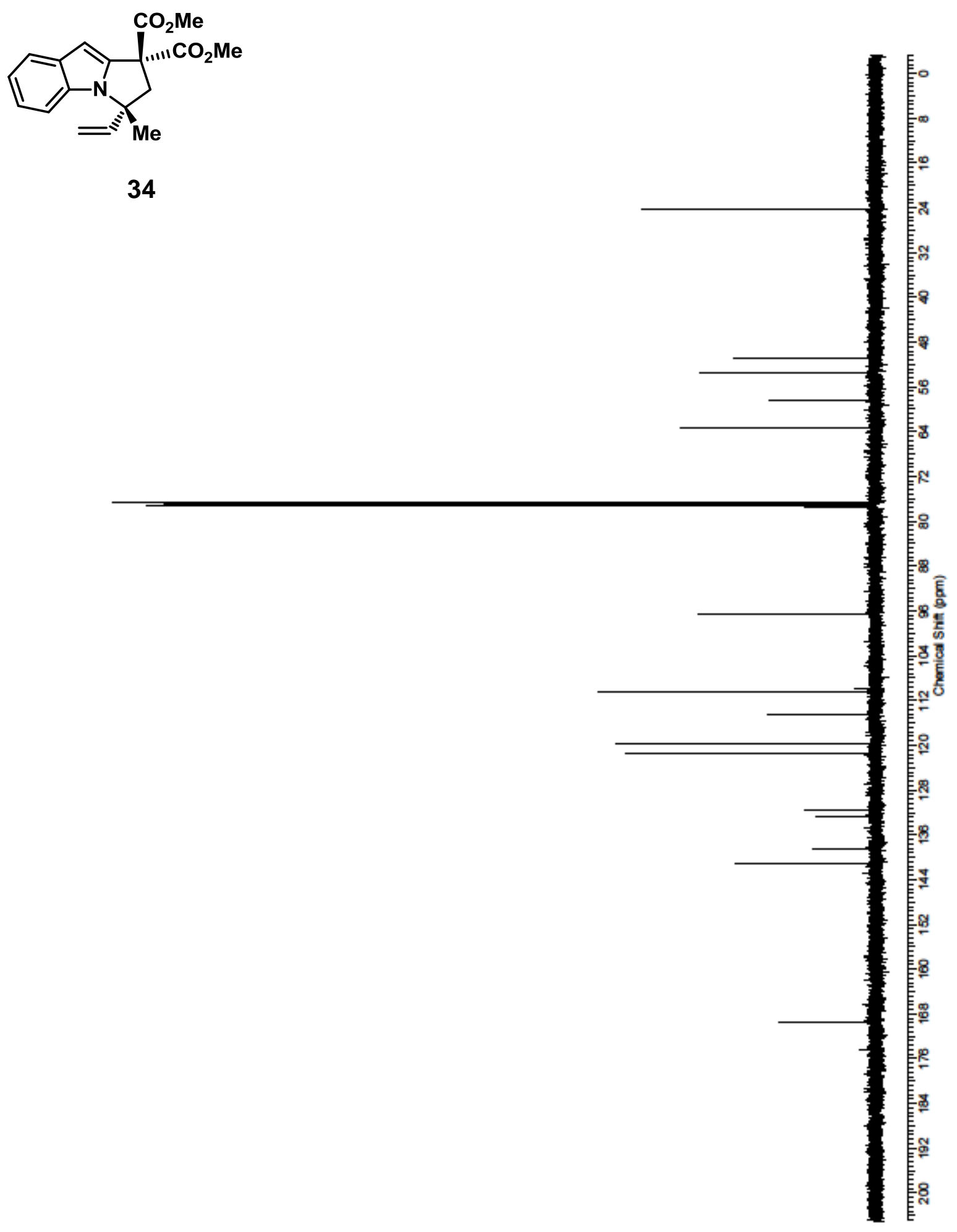




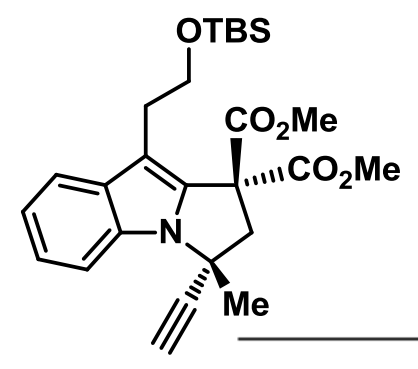

35

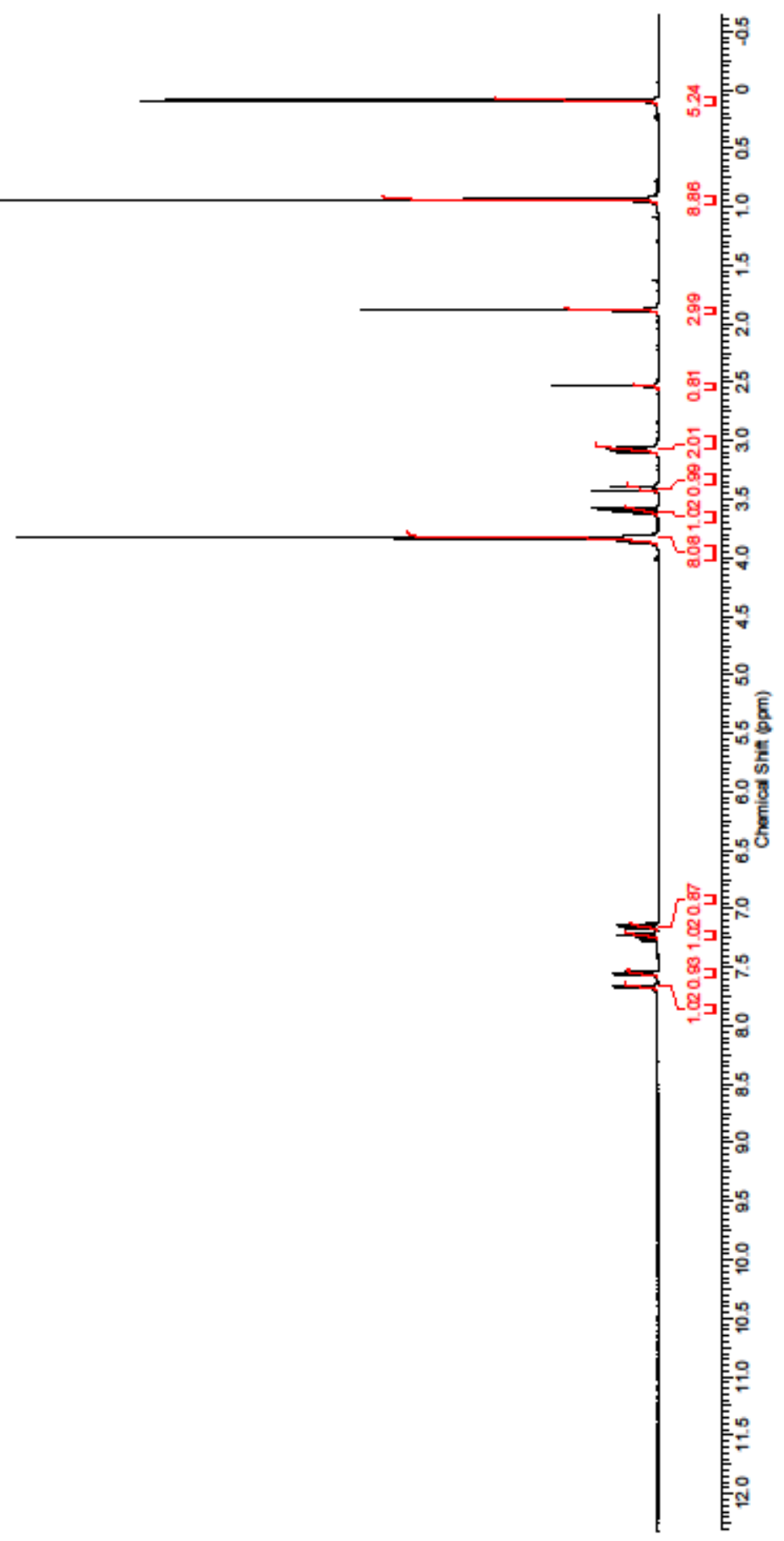




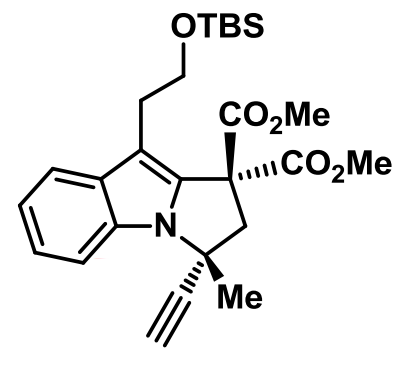

35

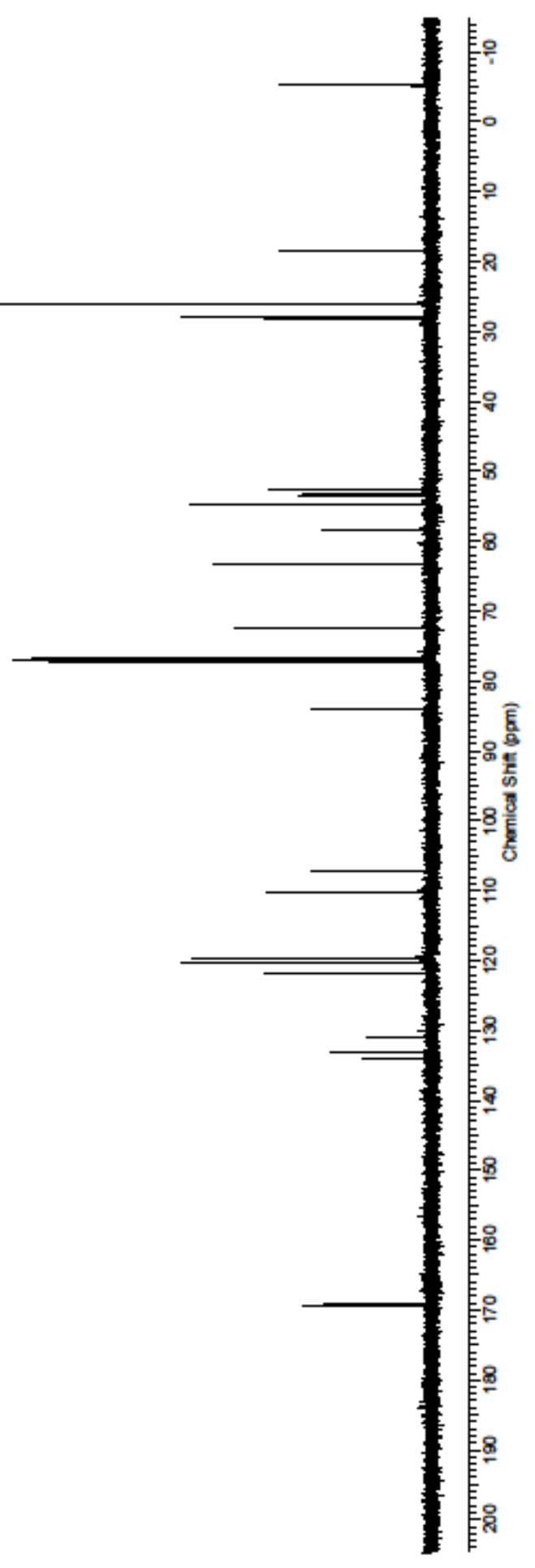




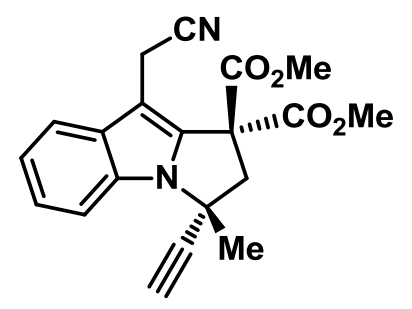

36

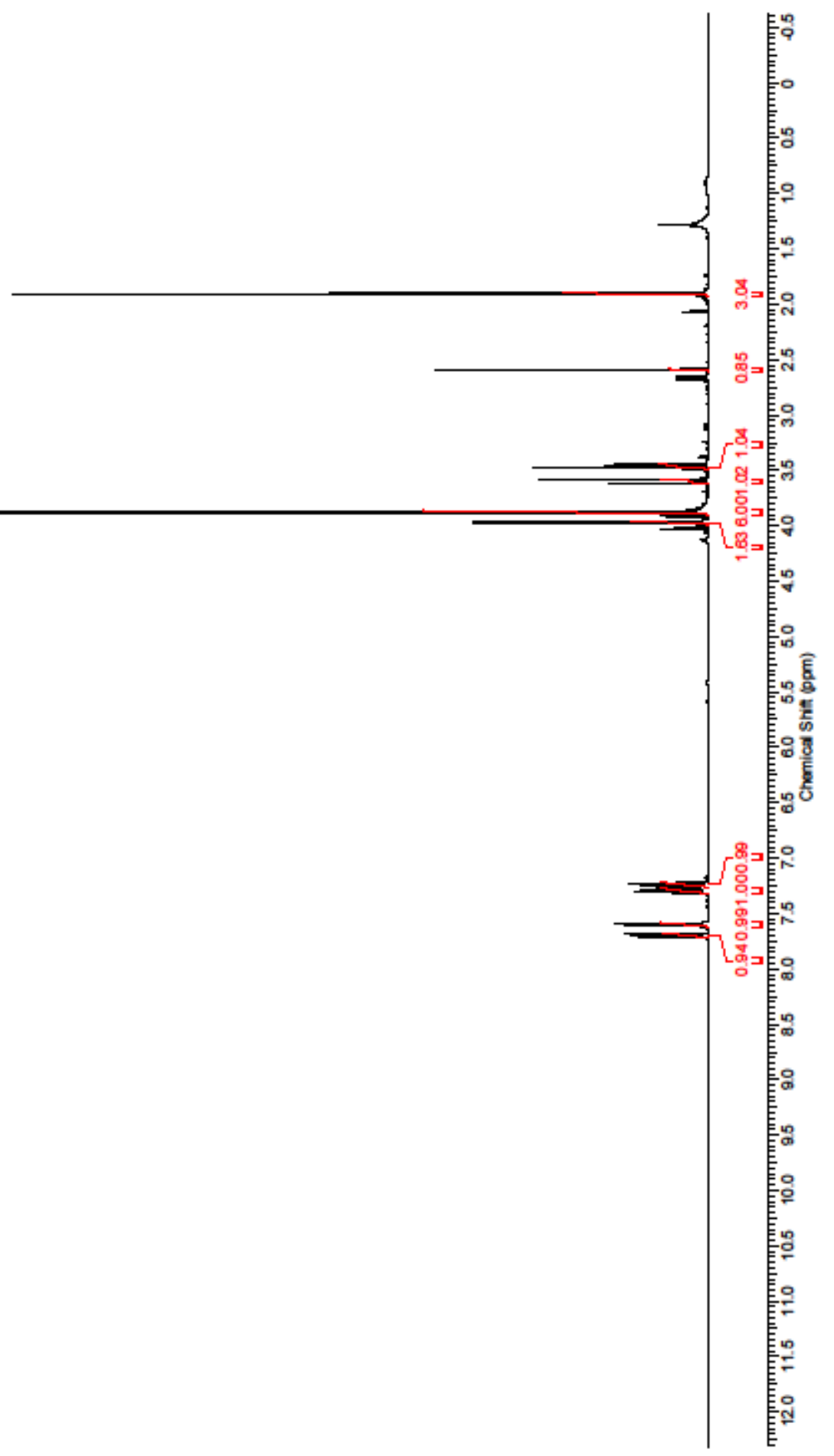




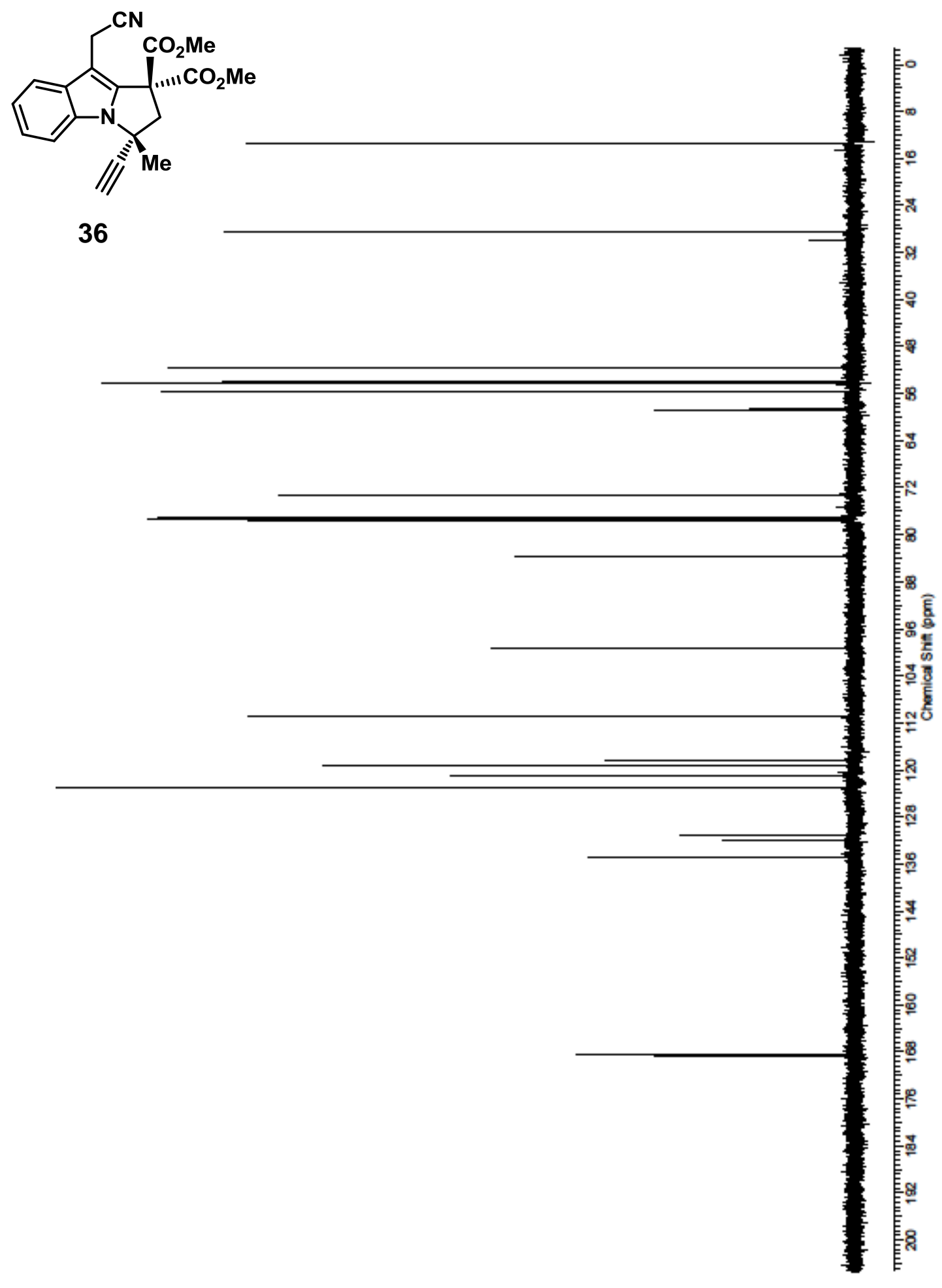




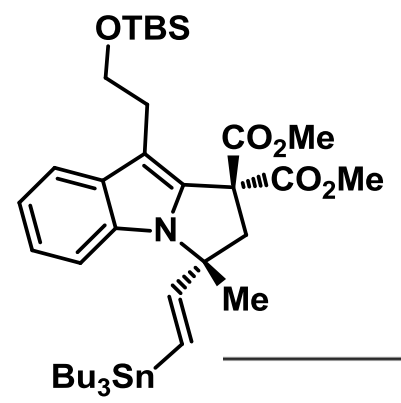

37

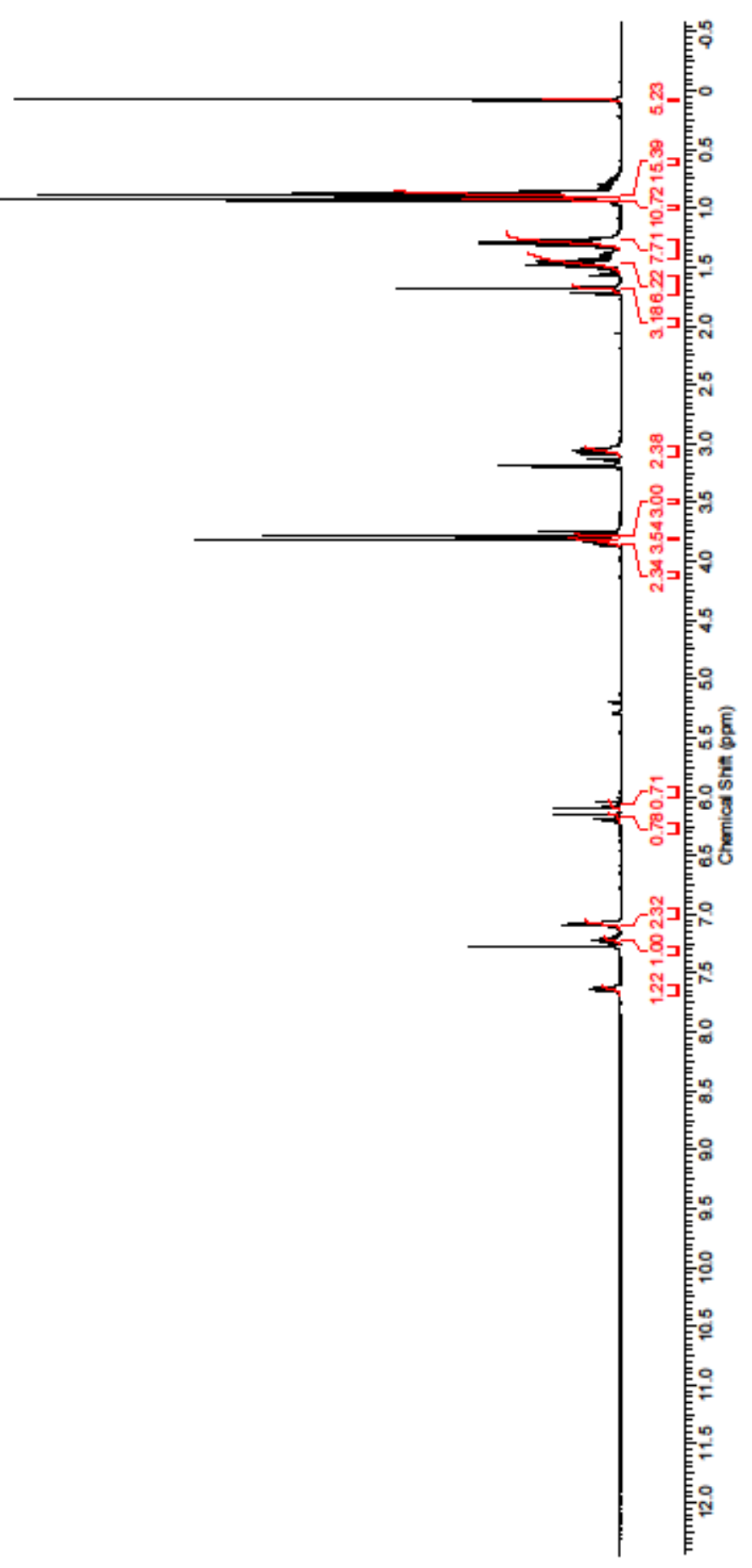



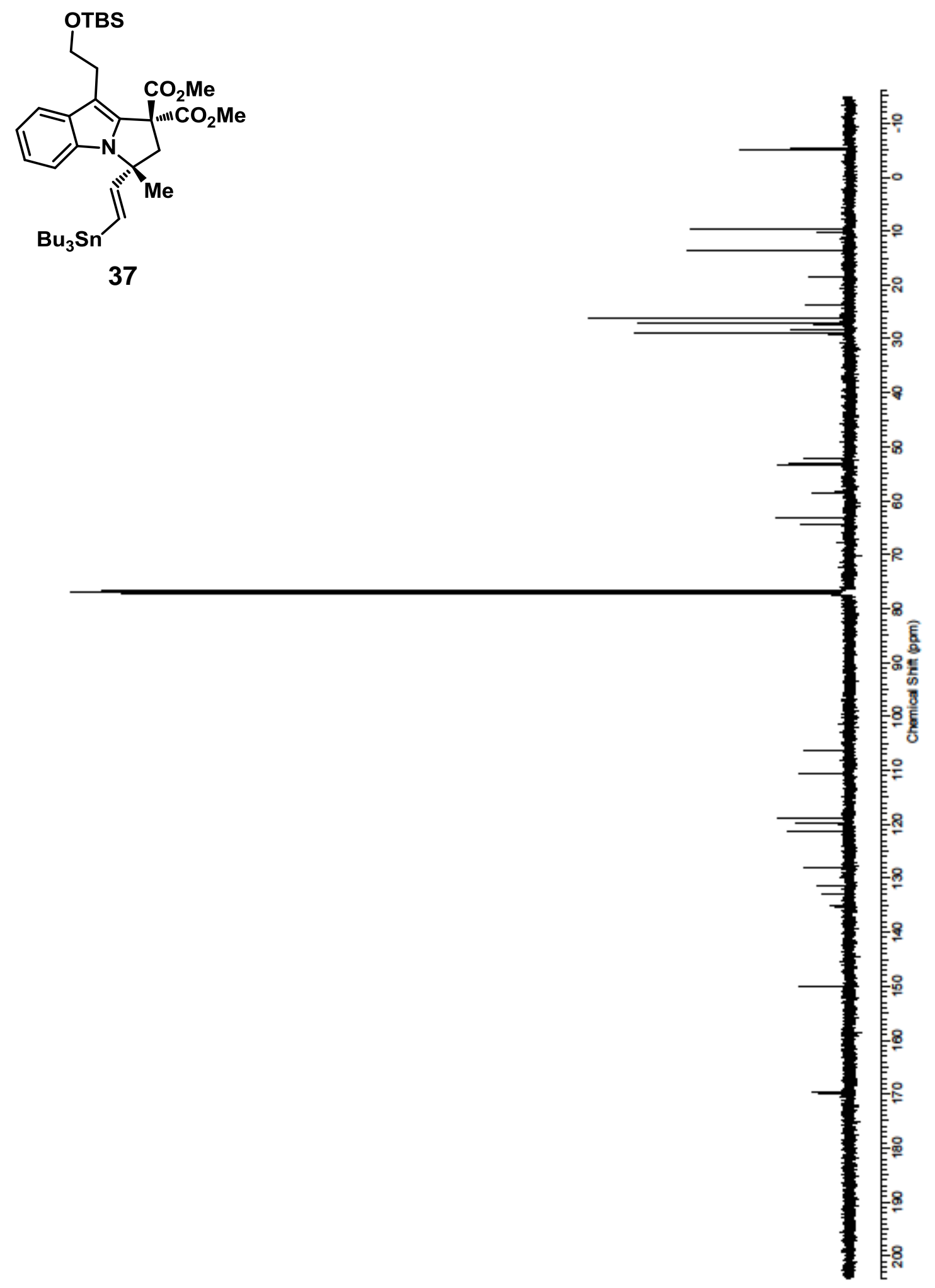


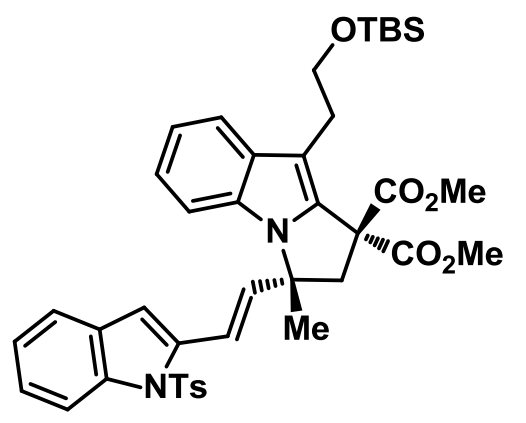

39

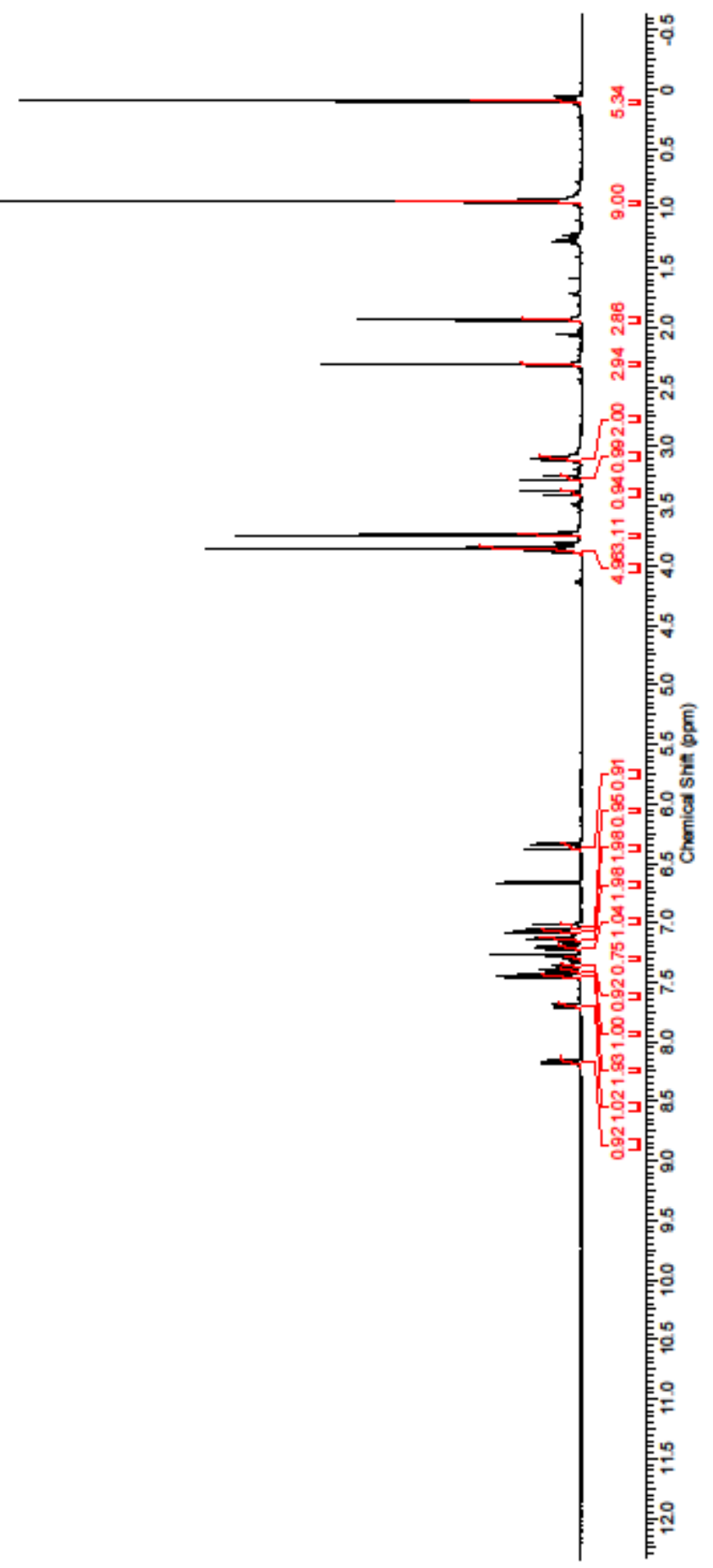



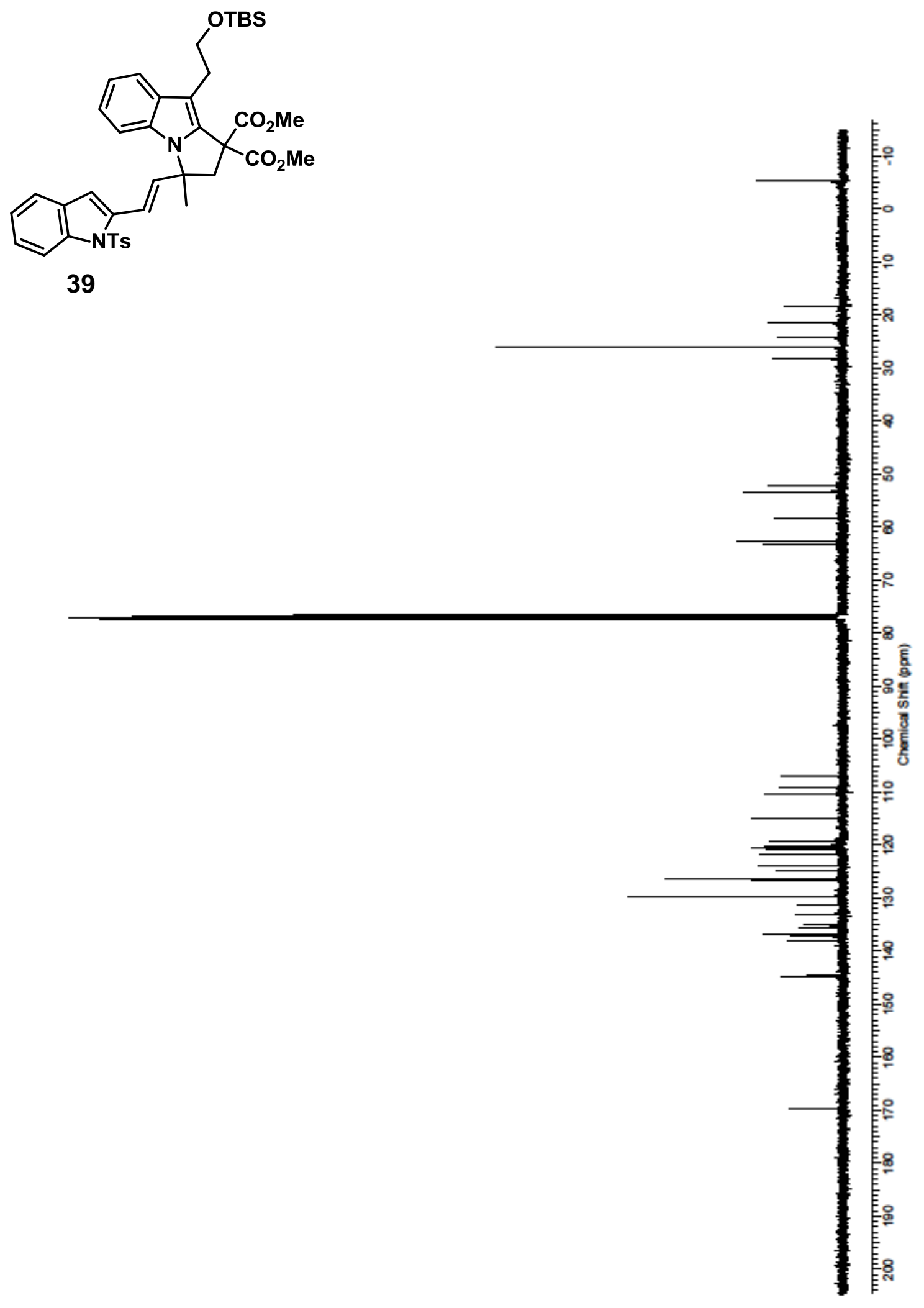